ITEP-M2/93円

ITFA $93-10$

March 1993

A.Morozov

\title{
INTEGRABILITY AND MATRIX MODELS
}

\begin{abstract}
The theory of matrix models is reviewed from the point of view of its relation to integrable hierarchies. Discrete 1-matrix, 2-matrix, "conformal" (multicomponent) and Kontsevich models are considered in some detail, together with the Ward identites ("W-constraints"), determinantal formulas and continuum limits, taking one kind of models into another. Subtle points and directions of the future research are also discussed.
\end{abstract}

Lectures presented at the University of Amsterdam and the Dutch Institute for High Energy Physics(NIKHEF)

in February-March 1993

\footnotetext{
${ }^{1}$ ITEP 117259 Moscow; morozov@vxdesy.desy.de
} 


\section{CONTENT}

1. Introduction

2. Ward identities for the simplest matrix models

2.1. Ward identites versus equations of motion

2.2. Virasoro constraints for the discrete 1-matrix model

2.3. CFT formulation of matrix models

2.4. Gross-Newman equation

2.5. Ward identities for Generalized Kontsevich Model

2.6. Discrete Virasoro constraints for the Gaussian Kontsevich model

2.7. Continuous Virasoro constraints for the $V=\frac{X^{3}}{3}$ Kontsevich model

2.8. $\tilde{W}$-constraints for the asymmetric 2-matrix model

2.9. $\tilde{W}$-constraints for generic 2 -matrix model

2.10. $\tilde{W}$-operators in Kontsevich model

3. Eigenvalue models

3.1. What are eigenvalue models

3.2. 1-matrix model

3.3. Itzykson-Zuber and Kontsevich integrals

3.4. Conventional Multimatrix models

3.5. Determinant formulas for eigenvalue models

3.6. Orthogonal polinomials

3.7. Two-component models in Miwa parametrization

3.8. Equivalence of the discrete 1-matrix and Gaussian Kontsevich models

3.9. Volume of unitary group

4. Integrable structure of eigenvalue models

4.1. The concept of integrability

4.2. The notion of $\tau$-function

4.3. $\tau$-functions, associated with free fermions

4.4. Basic determinant formula for the free-fermion correlator

4.5. Toda-lattice $\tau$-function and linear reductions of Toda-lattice hierarchy

4.6. Fermion correlator in Miwa coordinates

4.7. Matrix models versus $\tau$-functions.

4.8. String equations and generic concept of reduction

4.9. On the theory of GKM

4.10 1-Matrix model versus Toda-chain hierarchy

5. Continuum limits of discrete matrix models 
5.1. What is continuum limit

5.2. From Toda-chain to KdV

5.3. Double-scaling limit of 1-matrix model

5.4. From Gaussian to $X^{3}$-Kontsevich model

6. Conclusion

7. Acknowledgements

8. References 


\section{Introduction}

The purpose of these notes is to review one of the branches of modern string theory: the theory of matrix models with the emphasize on their intrinsic integrable structure. We begin with a brief description of the field and its place in the closest environement within entire string theory.

The main content of the string theory is the study of symmetries in the broadest possible sense of the word by methods of the quantum field theory. The usual scheme is to start from some symmetry and construct a field-theoretical model (usually 2-dimensional, for the reason that we do not discuss here), which possesses this symmetry in some simple sense (e.g. as Noether symmetry or as a chiral algebra). The main idea at this stage is to find a model which is exactly solvable (if nothing but the symmetry is given this is a nice principle to restrict dynamics). The next step is to study the hidden symmetries of the model, which are somehow responsible for its exact solvability and are usually much larger than the original symmetry.

This "inverse" step: model $\longrightarrow$ symmetry can be made at least with three different ideas in mind.

One can look for some hidden local (gauge) symmetry of the model, which is fixed or spontaneously broken, i.e. identify it with some other model which had more fields - auxiliary from the point of view of the smaller model and gauge - from that of the larger one. (Examples: gauged Wess-Zumino-Novikov- Witten (WZNW) model, topological theories in BRST formalism etc.)

One can take for a new (full) symmetry of the model just its operator algebra (algebra of observables) (see [2], [3] and also [4] for the first results in this direction). It deserves mentioning that gauging of entire algebra of observables gives rise to a "string field theory", associated with original model (considered as a string model).

One can construct effective action of the theory by exact evaluation of the functional integral.

As to direct step symmetry $\longrightarrow$ model, one can take as example the best understood case, when original symmetry is just a Lie algebra. Then the quantum mechanical model can be constructed by geometrical quantization technique (see [5] for the most important example of Kac-Moody algebra and the WZNW model).

\footnotetext{
${ }^{2}$ See [1] for a general review
} 
From the mathematical point of view the two elements of the above scheme look like algebra (theory of symmetries) and Analysis \& Geometry (field-theoretical models). The idea of constructing models with a given symmetry (and nothing else relevant for the dynamics) can be identified with the mathematical concept of "universal objects".

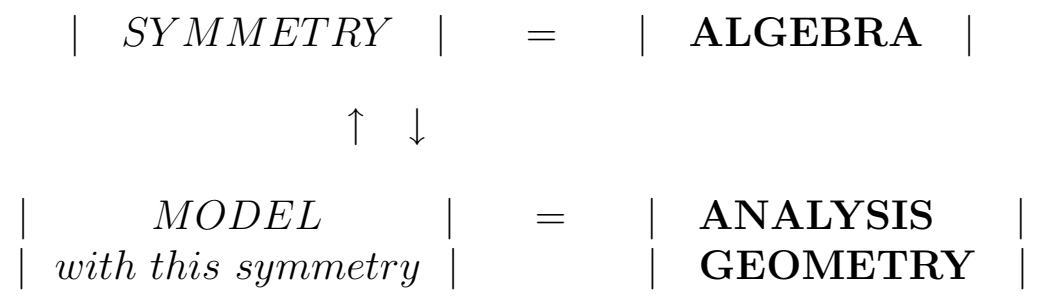

Theory of everything

The sequence of iterations of the two arrows in the picture leads to a deaper understanding, enlargening and generalization of all the notions involved: symmetry, exact solvability, field theory, geometrical structures, quantization etc, thus stimulating considerable progress both in physics and mathematics. If this iterative process can somehow converge, the limit point will deserve the name of the theory of everything, which will indeed unify all the possible field theoretical models by embedding them into a huge, but well structuired theory, which will be also exactly solvable in some, yet unspecified sense of the word. We refer to [1] for more details about this semiphilosophical programm, known under the name of (modern) string theory, and now turn to a more narrow subject: the theory of matrix models.

At the moment it is mainly associated with the theory of effective actions, at least so far this is where the main results of the modern theory of matrix models find their applications. This technique is especially suited for the study of effective actions, obtained after integration over 2-dimensional geometries (including the sum over genera) and it produces non-perturbative (exact) partition functions of particular string models. The main result of these studies points out that these partition functions exhibit two remarkable (though expected [6]) properties:

First, effective action for a given model is essentially the same as for any other model. In fact effective action is a function of coupling constants 
("sources" in the old-fashioned terms), which are nothing but coordinates in the space of various models (configuration space of entire string theory): variation of couplings change one model for another.

Second, effective action possesses a huge additional symmetry, which is somewhat similar to the general covariance in the space of all models (the above mentioned configuration space) and in the simplest examples, which have been studied so far, can be expressed in terms of integrable hierarchies. (This "general covariance" in the configuration space can after all turn into the main dynamical principle of the string theory.)

Both these features seem to be very general, arising whenever the largest possible Lagrangian with a given symmetry is considered (without restrictions on the possible counterterms, imposed by requirements of renormalizability or by locality-minimality "principles" - this is why this phenomenon is not widely known to field theorists). An example of highly-nontrivial calculations leading to similar conclusions can be found in ref. [7].

We hope that these remarks will become more clearer after some specific examples will be considered below. Still they deserve being fromulated in the full generality, not only to intrigue the reader, but also because they can serve for better understanding of the ideas and outcomes of generic string theory.

The "corner" of the string theory, associated with matrix models, can be described by the following picture: see Fig.1.

The big blocks within the body of string theory, which are directly related to matrix models are: theory of conformal models, that of the $N=2$ supersymmetry and the (loop-equation version of) the Yang-Mills theory (in any dimension). Also the Einstein gravity should be related to the subject in a way, similar to Yang-Mills theory, but these links are yet not clarified.

Both conformal theory and $N=2$ supersymmetry are sources of the concept of "topological models" [8]-[11]. These arise after gauging of all continuous symmetries of the WZNW models and/or as models with BRST-exact stress tensors, naturally appearing in the context of $N=2$ supersymmetry. If formulated in a self-consisted way in the "universal module space" (unification of module spaces of all finite-genus Riemann surfaces and bundles over them) these models turn into those of "topological gravity". Generating functionals of topological gravity models in fact generate infinite sequences 
of topological invariants of certain spaces (inverse definition is also possible in some cases [8], though the universal (generic) algorithm for the operation topology of some space $\longrightarrow$ topological gravity is not yet formulated).

Alternative models of $2 d$ quantum gravity arise straightforwardly from conformal models through a procedure of "summation over geometries". There are two essentially different approaches to the problem. One ("Polyakov approach") is to make use of the complex structure, intrinsic for conformal theory [12] and sum over Riemann surfaces, what involves integration over module spaces and sum over genera. The main technique used in this approach is the theory of free fields on Riemann surfaces [13], [14] and bosonization formalism for conformal field theories [15], [16]. This approach requires solution of Liouville theory, which still remains a problem under intensive investigation (in turn related to conformal field theory). Further progress in this direction should be related (or can be expressed in terms of) the adequate theory of the universal module space, handle-gluing operators etc. Similar objects arise in the field-theoretical approach to the topological gravity (see [17] for a recent review).

Alternative approach to summation over geometries does not refer at all to the complex structure and instead involves a sum over random equilateral triangulations [18-20]. 3 This is the place where matrix models first appear in the context of string theory. The random triangulation approach is by no means specific for conformal models (since it ignores the complex structure) and can be applied in many other situations - for example, to Yang-Mills (YM) theories in any dimensions (where instead of summation over geometries one needs "simply" to sum over ordinary Feynman diagramms).

Applications of the matrix-model method usually involve two steps: formulation and the study of "discrete" model and then taking its "continuum limit", giving rise to a new - "continuous matrix model", which sometimes can again be represented in a form of some matrix integral.

One of the main discoveries in the field of matrix models is that continuous

\footnotetext{
${ }^{3}$ Its relation to the Polyakov approach is a separate very interesting, important and badly understood problem, which allows a non-trivial reformulation in terms of number theory (see 21]). The main puzzle here is that equilateral triangulations are in fact arithmetic Riemann surfaces - a dense discrete subset in the entire module space, with interesting and deap algebraic properties. Equivalence of the two approaches to $2 d$ quantum gravity should imply the existence of some number-theoretical background behind the scene, which would be very nice to discover in full purity.
} 
models arising finally from the random-equilateral-triangulation description of the simplest (minimal with $c<1$ ) string models coincide with the simplest $\left(C P^{1}\right.$ Landau-Ginzburg) models of topological gravity [9], [22]-[24]: two (classes of) theories are identical (this is not yet proved in full detail, but is more than plausible).

So far continuous models are actually found and somehow understood only for string models, based on the $c<1$ minimal conformal theories (moreover, only for $q=1$ in the $(p, q)$-seria). Conformal models with $c \geq 1$, which are relevant for description of gauge theories in space-time dimension $d \geq 2$ (which possess particles, rather than only topological degrees of freedom), should give rise to the discrete matrix models with "non-factorizable" integration over "angular variables", of which the simplest (solvable) example is Kazakov-Migdal model [25]. The issue of continuum limit for such models is yet not understood (at least in terms of integrable structures, which should probably generalize the familiar theory of Toda hierarchies).

The goal of the study of matrix models is three-fold. First of all, one can look for the non-perturbative (exact) answers for the physical amplitudes in the given model. This is the subject which attracts most attention in the literature (for several obvious reasons). However, it is equally (and, perhaps, even more) important to understand the mathematical structure behind the matrix models (which involves topics like general theory of integrable hierarchies, geometrical quanization, Duistermaat-Heckman theorem ("localization theory") etc). Also important for the purposes of string theory is to use the results of the study of matrix models in order to unify a priori different models (according to the above mentioned principle: non-perturbative partition functions for different models differ by a change of variables in the space of coupling constants). Matrix models already played an important role in making this principle more clear and acceptable for many string theorists.

Let us make the next step and look even closer at the field of matrix models, especially, at its mostly studied domain, associated with the $d<2$ string models. Then the following structure will be seen: Fig.2.

The sample example of matrix model is that of 1-matrix integral

$$
Z_{N}\{t\} \equiv c_{N} \int_{N \times N} d H e^{\sum_{k=0}^{\infty} t_{k} \operatorname{Tr} H^{k}}
$$


where the integral is over $N \times N$ Hermitian matrix $H$ and $d H=\prod_{i, j} d H_{i j}$. There are three directions in which one can proceed starting from (1.1).

The first one [26] is to look for an invariant formulation of properties of the functional $Z_{N}\{t\}$. It appears to satisfy the infinite set of differential equations (in fact these are just Ward identities for the functional integral (1.1) [27]):

$$
\begin{aligned}
& L_{n} Z_{N}\{t\}=0, \quad n \geq-1, \\
& L_{n} \equiv \sum_{k=0}^{\infty} k t_{k} \frac{\partial}{\partial t_{k+n}}+\sum_{k=0}^{n} \frac{\partial^{2}}{\partial t_{k} \partial t_{n-k}}, \\
& \frac{\partial}{\partial t_{0}} Z_{N}=N Z_{N},
\end{aligned}
$$

which is known under the name of "discrete Virasoro constraints". $Z_{N}\{t\}$ can be represented as a correlator of screening operators in some auxiliary conformal model (of one free field on the "spectral surface"), and Virasoro constraints (1.2) are of course related to the Virasoro algebra in that conformal model. Also $Z_{N}\{t\}$ is some $\tau$-function of integrable "Toda-chain" hierarchy (in fact this statement should be a corollary of the Virasoro constraints, but this relation is still not very well understood).

The most straightforward further developement [26], [28] is to take the continuum limit of the Toda-chain hierarchy. In the specially adjusted ("doublescaling") limit [20]) it gives rise to the KdV-hierarchy, and the corresponding $\tau$-function appears subjected to the slightly different constraints 29, 28 (which again form a Borel subalgebra of some other "continuous Virasoro algebra"):

$$
\begin{aligned}
& \mathcal{L}_{2 n} \mathcal{Z}^{\text {cont }}\{T\}=0, \quad n \geq-1, \\
& \mathcal{L}_{2 n} \equiv \frac{1}{2} \sum_{\text {odd } k=1}^{\infty} k\left(T_{k}+r_{k}\right) \frac{\partial}{\partial T_{k+2 n}}+\frac{1}{4} \sum_{\text {odd } k=1}^{2 n-1} \frac{\partial^{2}}{\partial T_{k} \partial T_{2 n-k}}+ \\
& \quad+\frac{1}{16} \delta_{n, 0}+\frac{1}{4}\left(T_{1}+r_{1}\right)^{2} \delta_{n,-1},
\end{aligned}
$$

where $r_{k}=-\frac{2}{3} \delta_{k, 3}$. In fact

$$
\left.\mathcal{Z}^{\text {cont }}\{T\} \sim \lim _{\text {d.s. }\{N \rightarrow \infty\}} \sqrt{Z_{N}\{t\}}\right|_{t_{2 k+1}=0},
$$

and $T$ are related to $t$ by linear transformation [19],[28]:

$$
\begin{array}{r}
T_{k}=\frac{1}{2} \sum_{m \geq \frac{k-1}{2}} \frac{g_{m}}{\left(m-\frac{k-1}{2}\right) !} \frac{\Gamma\left(m+\frac{1}{2}\right)}{\Gamma\left(\frac{k}{2}+1\right)}, \quad k \text { odd } \\
g_{m}=m t_{2 m}, m \geq 1 ; \quad g_{0}=2 N .
\end{array}
$$


This $\mathcal{Z}^{\text {cont }}\{T\}$ can be again represented in the form of a matrix integral (over $n \times n$ Hermitian matrix) [22, [30, 33]:

$$
\mathcal{Z}^{\text {cont }}\{T\}=\mathcal{Z}_{V}\{T\}
$$

with $V(X)=\frac{X^{3}}{3}$, where

$$
\mathcal{Z}_{V}\{T\} \sim \mathcal{F}_{V, n}\{L\} \equiv \int_{n \times n} d X e^{-\operatorname{tr} V(X)+\operatorname{tr} L X}
$$

and

$$
T_{k}=\frac{1}{k} \operatorname{tr} L^{-k / 2}, \quad k \text { odd }
$$

The function $\mathcal{Z}_{V}\{T\}$ (but not $\mathcal{F}_{V, n}\{L\}$ ) is in fact independent of $n$ : the only thing that happens for finite values of $n$ is that the r.h.s. of (1.7) can not describe $\mathcal{Z}_{V}\{T\}$ at arbitrary points in the $T$-space, in accordance with (1.8). Continuous Virasoro constraints (1.3) are in fact equivalent to the trivial matrix-valued Ward identity

$$
\left(V^{\prime}\left(\frac{\partial}{\partial L_{\mathrm{tr}}}\right)-L\right) \mathcal{F}_{V, n}\{L\}=0 .
$$

Another direction to proceed from the discrete 1-matrix model is to rewrite it identically in the form of Kontsevich model: this time with $V(X)=$ $X^{2}$ and additional factor of $(\operatorname{det} X)^{N}$ aunder the integral in $\mathcal{F}_{V, n}\{L\}$ [56]. Then the double scaling limit can be studied in internal terms of Kontsevich models 36.

The third direction is towards multimatrix models. In continuous version they should provide $\tau$-functions of reduced KP-hierarchies [37] (KdV is the $p=2$ reduction), which are subjected to "continuous $W$-constraints" [29]. Matrix models of such $\tau$-functions are Kontsevich models with $V(X) \sim X^{p+1}$ [30]-33. At discrete level, however, things are not so simple. The most popular discrete multimatrix models [34] are defined as the multiple matrix integrals of the form:

$$
\begin{gathered}
Z_{N}\left\{t^{(\alpha)}\right\} \equiv \\
c_{N}^{p-1} \int_{N \times N} d H^{(1)} \ldots d H^{(p-1)} \prod_{\alpha=1}^{p-1} e^{\sum_{k=0}^{\infty} t_{k}^{(\alpha)} \operatorname{Tr} H_{(\alpha)}^{k}} \prod_{\alpha=1}^{p-2} e^{\operatorname{Tr} H^{(\alpha)} H^{(\alpha+1)}}
\end{gathered}
$$


(the form of the "interaction term" $\operatorname{Tr} H^{(\alpha)} H^{(\alpha+1)}$ is restricted by the "solvability" principle, but not unambiguously). In fact these models are particular examples of the "scalar-product eigenvalue models" and are not really distinguished except for the 1-matrix $(p=2)$ and 2-matrix $(p=3)$ cases. This is reflected in the absense of any reasonable Ward identites and integrable structures for these models, which would somehow involve their dependence on the variables $t^{(\alpha)}$ with $2 \leq \alpha \leq p-2$. Therefore the "multi-scaling continuum limit" of these models can hardly be investigated at any degree of rigourousness. (It is not so much important for "physical" applications to have any discrete models associated with the continuum ones, but this is an interesting problem for the "science for science".) For the 2-matrix $(p=3)$ case the Ward identities can be expressed in the form of " $\tilde{W}$-constraints" [38] and look like 30]

$$
\tilde{W}_{n-m}^{(m+1)}\{t\} Z_{N}\{t, \bar{t}\}=(-)^{m+n} \tilde{W}_{m-n}^{(n+1)}\{\bar{t}\} Z_{N}\{t, \bar{t}\}
$$

(here $t$ and $\bar{t}$ stand for $t^{(1)}$ and $t^{(2)}$, and $m, n$ are any non-negative integers).

The really interesting set of discrete multimatrix models does exist, but it is somewhat different from (1.10). These theories will be refered to as "conformal matrix models", since they arise straightforwardly as generalization of the "CFT-formulation" of the 1-matrix model [39]: it is enough to substitute discrete Virasoro constraints in the theory of one free field by the $W_{p}$-constraints in the theory of $p-1$ free fields. Matrix integral formulation then involves an "interaction term" $\operatorname{Det}\left(H^{(\alpha)} \otimes I-I \otimes H^{(\alpha+1)}\right)$ instead of $e^{\operatorname{Tr} H^{(\alpha)} H^{(\alpha+1)}}$, which is not very easy to guess a priori, but so defined models and their continuum limits can be examined in a manner, quite parallel to the one-matrix case (though not all is already done in this direction). Also this approach opens a possibility to formulate discrete models for any set of constraints, e.g. assosiated with the more exotic $W$ algebras and with quantum groups (i.e.can help to solve the inverse problem: constraints $\longrightarrow$ discrete matrix model). This is an option which also deserves further investigation. Another natural name for this set of theoris is "multi-component eigenvalue models".

Kontsevich models should be also related to topological models of LandauGinzburg gravity (LGG), though this relation is not yet clarified in full detail (see, however [40, [17]).

Among the main unresolved puzzles in this whole field is the description of 
generic $(p, q)$-models. Formally, Generalized Kontsevich model (1.7) provides this description, but in fact the partition function ( $\tau$-function) gets singular when the "phase transition" point where the $q$ changes is approached, and Kontsevich model with $V(X)=$ polinomial of degree $p+1$ provides a nice description only of $(p, 1)$-models. Generically, Kontsevich integral describes a duality transformation between $(p, q)$ and $(q, p)$ models: $(p, q) \longrightarrow(q, p)$ [41], but not any of these models separately. (The only exclusion are $(p, 1)$ models because they are related by Kontsevich transformation to the $(1, p)$ models which are completely trivial.)

In fact continuous models have two different sets of "time-variables". Thus far we introduced $T$, which are essentially expansion parameters of the generating functional for correlation functions. More exact, these parameters $\hat{T}$ depend on the particular model (vacuum), around which the perturbation expansion is performed, and they differ slightly from the model-independent $T$. Another set of "times", $r_{k}=\frac{p}{k(p-k)} \operatorname{Res}\left(V^{\prime}(\mu)\right)^{1-\frac{k}{p}} d \mu$, parametrizes the shape of the polinomial "potential" $V_{p}(X)$ (of degree $p+1$ ) and it describes the coordinates in the space of (matrix) models. These two types of variables - parameters of the generating functional and those labeling the shape of the Lagrangian - are almost the same (in fact they would be just the same, if there were no loop (quantum) effects). This similarity between T's and $r$ 's is reflected in the remarkable property of partition function of the $(p, 1)$ model - it essentially depends only on the sum of "times" $\hat{T}$ and $r$ [40]:

$$
\mathcal{Z}_{V_{p}}\{T\}=f_{p}\left(r \mid \hat{T}_{k}+r_{k}\right) \tau_{p}\left\{\hat{T}_{k}+r_{k}\right\}
$$

with some simple (and explicitly known) function $f_{p}$. (In eq.(1.8) above for monomial cubic potential $V_{3}(x)=\frac{x^{3}}{3}, \quad \hat{T}_{k}=T_{k}=\frac{1}{k} \operatorname{tr} L^{-k / 2}$, while $r_{k}=$ $\left.-\frac{2}{3} \delta_{k, 3} \cdot\right)$

The last thing to be mentioned in this general description of the field of the matrix model theory is their relation to group theory. Generalized Kontsevich model (1.7) is intimately connected to the "integrable nature" of group chracters and the coadjoint orbit integrals (characters of all the irreducible representations of $U(N)$ are usually $\mathrm{KP} \tau$-functions [101]). In fact some "discrete (or quantum) version" of Kontsevich integral is a sum over all unitary irreps of $U(n)$ ("integral" over a model of $U(n)$, or over the 
set of all coadjoint orbits):

$$
\mathcal{F}_{V}^{q u}\{G\} \equiv \sum_{R} d_{R} \chi_{R}(G) e^{-\sum_{k=0}^{\infty} v_{k} C_{k}(R)}
$$

where $d_{R}, \chi_{R}$ and $C_{k}(R)$ stand for dimension, character and the $k$-th Casimir of irreducible representation $R$ of $U(n)$. Time variables $T_{k} \sim \frac{1}{k} \operatorname{tr} G^{k}$, while

potential $V(X)=\sum_{k=0}^{\infty} s_{k} X^{k}$. This expression can be further generalized to

$$
\mathcal{F}_{V}^{q u}\{G\} \equiv \sum_{R} \chi_{R}(\bar{G}) \chi_{R}(G) e^{-\sum_{k=0}^{\infty} v_{k} C_{k}(R)} .
$$

Properties of these "quantum" Kontsevich models deserve further investigation (objects like (1.13) are also known to arise in the localization theory, in particular, in the study of the $d=2$ YM theory, see, for example, [43] and [44]).

These notes are essentially a review of the views and results of the group, working at Moscow (and Kiev). Since references will not be given every time, I present here the list of people involved into these investigations:

L.Chekhov, A.Gerasimov, A.Losev, S.Kharchev, Yu.Makeenko, A.Marshakov, A.Mikhailov, A.Mironov, A.Orlov, S.Pakuliak, I.Polyubin, A.Zabrodin.

I also apologize for the somewhat sporadic references to the works of other groups.

\section{Ward identities for the simplest matrix mod- els}

\subsection{Ward identites versus equations of motion}

We begin systematic consideration of matrix models from their simplest and at the same time the most basic property: the Ward identites (WI) for partition functions. Partition function is by definition a functional of the coupling constants in the Lagrangian and WI will be understood here as (differentiual or finite-difference) equations, imposed on this functional. If 
partition function is represented in the form of a matrix integral, the WI are usually implied by its invariance (or, better to say - covariance) under the change of the integration variables (thus the name "WI").

In ordinary field theory we are usually dealing with models, where WI either do not exist at all, or at most there is finite number of them - then they are interpreted as reflecting the symmetry of the theory. However, by no means the finite set of these WI provides a complete description of dynamics of the theory: the number of (quantum) equations of motion (EqM) is usually infinite and their solutions are never fixed by the WI. In fact this difference between WI and EqM arises because the Lagrangians, considered in the ordinary field theory are not of the most general form: they are usually severely restricted by "principles" like renormalizability or minimality. Because of this there is simply not many enough coupling constants in the Lagrangian to describe the result of any variation of integration variables as that of the variation of coupling constants, and thus not every equation of motion can be represented as (differential) equation for the partition function. In other words, by restricting the shape of Lagrangian for "non-symmetric" reasons one breaks the original huge "symmetry" (covariance) of the model, which was enough to describe all the dynamics (all EqM) as dictated by symmetry,- and a broader view is necessary in order to recognize EqM as the WI, associated with that original high symmetry. This symmetry (it is not of Noether type, of course) is peculiar property of all the quantum mechanical partition functions, since these usually arise from the procedure of functional integration.

It happened so that matrix models appeared to be the first class of quantum-mechanical systems (functional integrals) for which this identity:

$$
\text { all } \mathrm{EqM} \equiv \text { all WI }
$$

\footnotetext{
${ }^{4}$ To avoid confusion we emphasize that such representation does not need to exist, at least in any simple form. The more the theory of matrix models develops, the less it has to do with matrices and matrix integrals. However (as in the case of entire string theory) original name has a tendency to survive. Anyhow, the main content of the theory of matrix models (at least of its branch, analyzed in these notes) is the search for invariant formulations of the properties of partition functions, while matrix integrals (if at all existing) are considered as their particular realizations (representations). Moreover, there can exist very different matrix integral representations of the same partition function, the simplest example being just the basic discrete 1-matrix model, which can be also represented in the form of Kontsevich integral (see below).
} 
was not simply observed as a curious phenomenon, but became a subject of intensive investigation and is identified as the source of exact solvability (integrability) of the theory. Of course, significance of this observation (and its implications) is quite universal, by no means restricted to the field of matrix models themselves, however, it is yet not enough appreciated by the experts in other fields. In any case, we are going to deal only with matrix models in these notes.

We proceed to consideration of the WI according to the following plan (not all the arrows will be actually discussed): see Fig.3.

\subsection{Virasoro constraints for the discrete 1-matrix model}

The basic example [26, 27] which illustrates the arguments from the previous subsection is provided by the 1-matrix model

$$
Z_{N}\{t\} \equiv c_{N} \int_{N \times N} d H e^{\sum_{k=0}^{\infty} t_{k} \operatorname{Tr} H^{k}} .
$$

This integral is invariant under any change of variables $H \rightarrow f(H)$. It is convenient to choose the special basis in the space of such transformations:

$$
\delta H=\epsilon_{n} H^{n+1} .
$$

Here $\epsilon_{n}$ is some infinitesimal matrix and, of course, $n \geq-1$. The value of integral can not change under the change of integration variable, and we obtain the identity:

$$
\int_{N \times N} d H e^{\sum_{k=0}^{\infty} t_{k} \operatorname{Tr} H^{k}}=\int d\left(H+\epsilon_{n} H^{n+1}\right) e^{\sum_{k=0}^{\infty} t_{k} \operatorname{Tr}\left(H+\epsilon_{n} H^{n+1}\right)^{k}},
$$

i.e.

$$
\int d H e^{\sum_{k=0}^{\infty} t_{k} \operatorname{Tr} H^{k}}\left(\sum_{k=0}^{\infty} k t_{k} \operatorname{Tr} H^{k+n}+\operatorname{Tr} \frac{\delta H^{n+1}}{\delta H}\right) \equiv 0 .
$$

In order to evaluate the Jacobian $\operatorname{Tr} \frac{\delta H^{n+1}}{\delta H}$ let us restore the matrix indices:

$$
\left(\delta H^{n+1}\right)_{i j}=\sum_{k=0}^{n}\left(H^{k} \delta H H^{n-k}\right)_{i j}=\sum_{k=0}^{n}\left(H^{k}\right)_{i l}(\delta H)_{l m}\left(H^{n-k}\right)_{m j} .
$$


In $\operatorname{Tr} \frac{\delta H^{n+1}}{\delta H}$ one should take $l=i$ and $m=j$, so that

$$
\operatorname{Tr} \frac{\delta H^{n+1}}{\delta H}=\sum_{k=0}^{n} \operatorname{Tr} H^{k} \operatorname{Tr} H^{n-k} .
$$

Now we can note that since we started from Lagrangian of the most general form (consistent with the symmetry $H \rightarrow U H U^{\dagger}$ ), any correlation function can be obtained as variation of the coupling constants (all possible sources are included as counterterms). In our particular example this is just a trivial remark:

$$
\begin{aligned}
<\operatorname{Tr} H^{a_{1}} \ldots \operatorname{Tr} H^{a_{n}}> & =\int d H e^{\sum_{k=0}^{\infty} t_{k} \operatorname{Tr} H^{k}} \operatorname{Tr} H^{a_{1}} \ldots \operatorname{Tr} H^{a_{n}}= \\
& =\frac{\partial^{n}}{\partial t_{a_{1}} \ldots \partial t_{a_{n}}} Z_{N}\{t\} .
\end{aligned}
$$

We can use this relation together with (2.4) in order to rewrite (2.3) as:

$$
L_{n} Z_{N}\{t\}=0, \quad n \geq-1
$$

with

$$
L_{n} \equiv \sum_{k=0}^{\infty} k t_{k} \frac{\partial}{\partial t_{k+n}}+\sum_{k=0}^{n} \frac{\partial^{2}}{\partial t_{k} \partial t_{n-k}} .
$$

Note that according to the definition (2.1)

$$
\frac{\partial}{\partial t_{0}} Z_{N}=N Z_{N}
$$

Several remarks are now in order.

First of all, expression in brackets in (2.3) represents just all the equations of motion for the model (2.1), and (2.6) is nothing but another way to represent the same set of equations. This is an example of the above-mentioned identification of EqM and WI.

Second, commutator of any two operators $L_{n}$ apearing in (2.6) should also annihilate $Z_{N}\{t\}$. It is another indication (not a convincing one, however) that we already got a complete set of constraints, that $L_{n}$ 's form a closed (Virasoro) algebra:

$$
\left[L_{n}, L_{m}\right]=(n-m) L_{n+m}, \quad n, m \geq-1 .
$$


Third, (2.6) can be considered as invariant formulation of what is $Z_{N}$ : it is a solution of this set of compatible differential equations. From this point of view eq.(2.1) is rather a particular representation of $Z_{N}$ and it is sensible to look for other representations as well (we shall later discuss two of them: one in terms of CFT, another in terms of Kontsevich integrals).

Fourth, one can try to analyze the uniqueness of the solutions to (2.6). If there are not too many of them the set of constraints can be considered complete. A natural approach to classification of solutions to the algebra of constraints is in terms of the orbits of the corresponding group [45]. Let us consider an oversimplified example, which can still be usefull to understand implications of the complete set of WI as well as clarify the meaning of classes of universality and of integrability.

Imagine, that instead of (2.6) with $L_{n}$ 's defined in (2.7) we would obtain somewhat simpler equations: 色

$$
l_{n} Z=0, \quad n \geq 0 \quad \text { with } l_{n}=\sum_{k=1}^{\infty} k t_{k} \frac{\partial}{\partial t_{k+n}} .
$$

Then operator $l_{1}$ can be interpreted as generating the shifts

$$
\begin{gathered}
t_{2} \longrightarrow t_{2}+\epsilon_{1} t_{1}, \\
t_{3} \longrightarrow t_{3}+2 \epsilon_{1} t_{2},
\end{gathered}
$$

We can use it to shift $t_{2}$ to zero, and eq. $l_{1} Z=0$ then implies that

$$
Z\left(t_{1}, t_{2}, t_{3}, \ldots\right)=Z\left(t_{1}, 0, \tilde{t}_{3}, \ldots\right)
$$

$\left(\tilde{t}_{k}=t_{k}-\frac{(k-1) t_{2} t_{k-1}}{t_{1}}, k \geq 3\right)$.

Next, operator $l_{2}$ generates the shifts

$$
\begin{gathered}
t_{3} \longrightarrow t_{3}+\epsilon_{2} t_{1}, \\
t_{4} \longrightarrow t_{4}+2 \epsilon_{2} t_{2},
\end{gathered}
$$

\footnotetext{
${ }^{5}$ One can call them "classical" approximation to (2.6), since they would arise if the variation of measure (i.e. a "quantum effect") was not taken into account in the derivation of (2.6). Though this concept is often used in physics it does not have much sense in the present context, when we are analyzing exact properties of functional (matrix) integrals.
} 
and does not affect $t_{2}$. We can now use eq. $l_{2} Z=0$ to argue that

$$
Z\left(t_{1}, t_{2}, t_{3}, t_{4}, \ldots\right)=Z\left(t_{1}, 0, \tilde{t}_{3}, \tilde{t}_{4}, \ldots\right)=Z\left(t_{1}, 0,0, \tilde{\tilde{t}}_{4}, \ldots\right)
$$

etc. Assuming that $Z$ is not very much dependent on $t_{k}$ with $k \rightarrow \infty$, 可 we can conclude, that

$$
Z\left(t_{1}, t_{2}, t_{3}, \ldots\right)=Z\left(t_{1}, 0,0, \ldots\right)=Z(1,0,0, \ldots)
$$

(at the last step we also used the equation $l_{0} Z=0$ to rescale $t_{1}$ to unity).

All this reasoning was correct provided $t_{1} \neq 0$. Otherwise we would get $Z(0,1,0,0, \ldots)$, if $t_{1}=0, t_{2} \neq 0$, or $Z(0,0,1,0, \ldots)$, if $t_{1}=t_{2}=0, t_{3} \neq 0$ etc. In other words, we obtain classes of universality (such that the value of partition function is just the same in the whole class), which in this oversimplified example are labeled just by the first non-vanishing time-variable. Analysis of the orbit structure for the actually important realizations of groups, like that connected to eq.(2.7) has never been performed in the context of matrix model theory. It may deserve emphasizing that the constraints, as we saw, can actually allow one to eliminate (solve exactly) all the dependence on the time-variables, in less trivial examples they somehow imply the integrability structure, which is just a slightly more complicated version of the same solvability phenomenon.

\subsection{CFT formulation of matrix models}

Given a complete set of the constraints on partition function of infinitely many variables which form some closed algebra we can now ask an inverse question: how these equations can be solved or what is the integral representation of partition function. One approach to this problem is analysis of orbits, briefly mentioned at the end of the previous section. Now we turn to another technique [39], which makes use of the knowledge from conformal field theory. This constructions can have some meaning from the "physical" point of view, which implies certain duality between the 2-dimensional world surfaces and the spectral surfaces, associated to configuration space of the string theory. However, our goal now is more formal: to use the means of CFT for solution of the constraint equations.

\footnotetext{
${ }^{6}$ This, by the way, is hardly correct in this particular example, when the group has no compact orbits.
} 
This is very natural in the case when the algebra of constraints is Virasoro algebra, as in the case of the 1-matrix model, or some other algebra if it is known to arise naturally as chiral algebra in some simple conformal models. In fact the approach which will be now discussed is rather general and can be applied to construction of matrix models, associated with many different algebraic structures.

We begin from the set of equations (2.6) which we shall further refer to as "discrete Virasoro constraints". The CFT formulation of interest should provide the solution to these equations in the form of some correlation function in some conformal field theory. Of course, it becomes natural, if we somehow identify the operators $L_{n}$, which form Virasoro algebra with the harmonics of the stress-tensor $T_{n}$, which satisfy the same algebra, and manage to relate the constraint that $L_{n}$ annihilate the correlator to the statement that $T_{n}$ annihilate the vacuum state. Thus the procedure is naturally split into two steps: First we should find a $t$-dependent operator ("Hamiltonian") $H(t)$, such that

$$
L_{n}(t)\left\langle e^{H(t)} \ldots=\left\langle e^{H(t)} T_{n} \ldots\right.\right.
$$

This will relate differential operators $L_{n}$ to $T_{n}$ 's expressed through the fields of conformal model. Second we need to enumerate the states, that are annihilated by the operators $T_{n}$ with $n \geq-1$, i.e. solve equation

$$
T_{n}|G\rangle=0
$$

for the ket-states, what is an internal problem of conformal field theory. If both ingredients $H(t)$ and $|G\rangle$ are found, solution to the problem is given by

$$
\left\langle e^{H(t)} \mid G\right\rangle .
$$

To be more explicit, for the case of the discrete Virasoro constraints we can just look for solutions in terms of the simplest possible conformal model: that of a one holomorphic scalar field

$$
\begin{array}{r}
\phi(z)=\hat{q}+\hat{p} \log z+\sum_{k \neq 0} \frac{J_{-k}}{k} z^{k} \\
{\left[J_{n}, J_{m}\right]=n \delta_{n+m, 0}, \quad[\hat{q}, \hat{p}]=1 .}
\end{array}
$$


Then the procedure is as follows: Define vacuum states

$$
\begin{aligned}
J_{k}|0\rangle & =0, \quad & \langle N| J_{-k}=0, \quad k>0 \\
\hat{p}|0\rangle & =0, \quad & \langle N| \hat{p}=N\langle N|,
\end{aligned}
$$

the stress-tensor

$$
T(z)=\frac{1}{2}[\partial \phi(z)]^{2}=\sum T_{n} z^{-n-2}, \quad T_{n}=\sum_{k>0} J_{-k} J_{k+n}+\frac{1}{2} \sum_{\substack{a+b=n \\ a, b \geq 0}} J_{a} J_{b},
$$

and the Hamiltonian

$$
\begin{aligned}
& H(t)=\frac{1}{\sqrt{2}} \sum_{k>0} t_{k} J_{k}=\frac{1}{\sqrt{2}} \oint_{C_{0}} U(z) J(z) \\
& U(z)=\sum_{k>0} t_{k} z^{k}, \quad J(z)=\partial \phi(z) .
\end{aligned}
$$

It can be easily checked now that

$$
L_{n}\langle N| e^{H(t)} \ldots=\langle N| e^{H(t)} T_{n} \ldots
$$

and

$$
T_{n}|0\rangle=0, \quad n \geq-1
$$

As an immediate consequence, any correlator of the form

$$
Z_{N}\{t \mid G\}=\left\langle N\left|e^{H(t)} G\right| 0\right\rangle
$$

gives a solution to (2.6) provided

$$
\left[T_{n}, G\right]=0, \quad n \geq-1 .
$$

In fact operators $G$ that commute with the stress tensor are well known: these are just any functions of the "screening charges" $\square$

$$
Q_{ \pm}=\oint J_{ \pm}=\oint e^{ \pm \sqrt{2} \phi}
$$

The correlator (2.18) will be non-vanishing only if the matching condition for zero-modes of $\phi$ is satisfied. If we demand the operator to depend only

\footnotetext{
${ }^{7}$ For notational simplicity we omit the normal ordering signs, in fact the involved operators are $: e^{H}:$ and $: e^{ \pm \sqrt{2} \phi}$ :
} 
on $Q_{+}$, this implies that only one term of the expansion in powers of $Q_{+}$ will contribute to (2.18), so that the result is essentially independent on the choice of the function $G\left(Q_{+}\right)$, we can for example take $G\left(Q_{+}\right)=e^{Q_{+}}$and obtain:

$$
Z_{N}\{t\} \sim \frac{1}{N !}\left\langle N\left|e^{H(t)}\left(Q_{+}\right)^{N}\right| 0\right\rangle .
$$

This correlator is easy to evaluate using the Wick theorem and the propagator $\phi(z) \phi\left(z^{\prime}\right) \sim \log \left(z-z^{\prime}\right)$ and finally we get

$$
\begin{aligned}
Z_{N}\{t\} & =\frac{1}{N !}\left\langle N\left|: e^{\frac{1}{\sqrt{2}} \oint_{C_{0}} U(z) \partial \phi(z)}: \prod_{i=1}^{N} \oint_{C_{i}} d z_{i}: e^{\sqrt{2} \phi\left(z_{i}\right)}:\right| 0\right\rangle= \\
& =\frac{1}{N !} \prod_{i=1}^{N} \oint_{C_{i}} d z_{i} e^{U\left(z_{i}\right)} \prod_{i<j}^{N}\left(z_{i}-z_{j}\right)^{2}
\end{aligned}
$$

in the form of a multiple integral, which can in fact be directly related to the matrix integral in (2.1), see [46] and the next section.

Thus in the simplest case we resolved the inverse problem: reconstructed the integral representation from the set of discrete Virasoro constraints. However, the answer we got seems a little more general than (2.1): the r.h.s. of eq. (2.22) still depends on the contours of integration. Moreover, we can also recall that the operator $G$ above could depend not only on $Q_{+}$, but also on $Q_{-}$. The most general formula is a little more complicated than (2.22):

$$
\begin{gathered}
Z_{N}\left\{t \mid C_{i}, C_{r}\right\} \sim \frac{1}{(N+M) ! M !}\left\langle N\left|e^{H(t)}\left(Q_{+}\right)^{N+M}\left(Q_{-}\right)^{M}\right| 0\right\rangle= \\
=\frac{1}{(N+M) ! M !} \prod_{i=1}^{N+M} \oint_{C_{i}} d z_{i} e^{U\left(z_{i}\right)} \prod_{r=1}^{M} \oint_{C_{r}^{\prime}} d z_{r}^{\prime} e^{U\left(z_{r}^{\prime}\right) .} \\
\cdot \frac{\prod_{i<j}^{N+M}\left(z_{i}-z_{j}\right)^{2} \prod_{r<s}^{N}\left(z_{r}^{\prime}-z_{s}^{\prime}\right)^{2}}{\prod_{i}^{N+M} \prod_{r}^{M}\left(z_{i}-z_{r}\right)^{2}} .
\end{gathered}
$$

We refer to the papers [39] for discussion of the issue of the contour-dependence. In certain sense all these different integrals can be considered as branches of the same analytical function $Z_{N}\{t\}$. Dependence on $M$ is essentially eliminated by Cauchy integration around the poles in denominator in (2.23).

Above construction can be straightforwardly applied to other algebras of constraints, provided:

(i) The free-field representation of the algebra is known in the CFTframework, such that the generators are polinomials in the fields $\phi$ (only in 
such case it is straightforward to construct a Hamiltonian $H$, which relates CFT-realization of the algebra to that in terms of differential operators w.r.to the $t$-variables; in fact under this condition $H$ is usually linear in $t$ 's and $\phi ' s)$. There are examples (like Frenkel-Kac representation of level $k=1$ simply-laced Kac-Moody algebras 47 or generic reductions of the WZNW model [16], 48]-[51]) when generators are exponents of free fields, then this construction should be slightly modified.

(ii) It is easy to find vacuum, annihilated by the relevant generators (here, for example, is the problem with application of this approach to the case of "continuous" Virasoro and $W$-constraints). The resolution to this problem involves consideration of correlates on Riemann surfaces with non-trivial topologies, often - of infinite genus.

(iii) The free-field representation of the "screening charges", i.e. operators that commute with the generators of the group within the conformal model, is explicitly known.

These conditions are fulfilled in many case in CFT, including conventional $\mathbf{W}$-algebras [52 and $\mathcal{N}=1$ supersymmetric models [53].

For illustration purposes we present here several formulas from the last paper of ref. [39] for the case of the $\mathbf{W}_{r+1}$-constraints, associated with the simply-laced algebras $\mathcal{A}$ of rank $r$.

Partition function in such "conformal multimatrix model" is a function of "time-variables" $t_{k}^{(\lambda)}, k=0 \ldots \infty, \lambda=1 \ldots r=\operatorname{rank} \mathcal{A}$, and also depends on the integer-valued $r$-vector $\boldsymbol{N}=\left\{N_{1} \ldots n_{r}\right\}$. The $\mathbf{W}_{r+1}$-constraints imposed on partition function are:

$$
W_{n}^{(a)}(t) Z_{N}^{\mathcal{A}}\{t\}=0, \quad n \geq 1-a, \quad a=2 \ldots r+1 .
$$

The form of the $W$-operators is somewhat complicated, for example, in the case of $r+1=3$ (i.e. for $\mathcal{A}=A_{2}(S L(3))$ )

$$
\begin{aligned}
W_{n}^{(2)} & =\sum_{k=0}^{\infty}\left(k t_{k} \frac{\partial}{\partial t_{k+n}}+k \bar{t}_{k} \frac{\partial}{\partial \bar{t}_{k+n}}\right)+ \\
& +\sum_{a+b=n}\left(\frac{\partial^{2}}{\partial t_{a} \partial t_{b}}+\frac{\partial^{2}}{\partial \bar{t}_{a} \partial \bar{t}_{b}}\right)
\end{aligned}
$$

\footnotetext{
${ }^{8}$ In the case of $\mathcal{N}=2$ supersymmetry a problem arises because of the lack of reasonable screening charges. At the most naive level the relevant operator to be integrated over superspace (over $d z d^{\mathcal{N}} \theta$ ) in order to produce screening charge has dimension $1-\frac{1}{2} \mathcal{N}$, which vanishes when $\mathcal{N}=2$.
} 


$$
\begin{gathered}
W_{n}^{(3)}=\sum_{k, l>0}\left(k t_{k} l t_{l} \frac{\partial}{\partial t_{k+n+l}}-k \bar{t}_{k} l \bar{t}_{l} \frac{\partial}{\partial t_{k+n+l}}-2 k t_{k} l \bar{t}_{l} \frac{\partial}{\partial \bar{t}_{k+n+l}}\right)+ \\
+2 \sum_{k>0}\left[\sum_{a+b=n+k}\left(k t_{k} \frac{\partial^{2}}{\partial t_{a} \partial t_{b}}-k t_{k} \frac{\partial^{2}}{\partial \bar{t}_{a} \partial \bar{t}_{b}}-2 k \bar{t}_{k} \frac{\partial^{2}}{\left.\partial t_{a} \partial \bar{t}_{b}\right)}\right]+\right. \\
+\frac{4}{3} \sum_{a+b+c=n}\left(\frac{\partial^{3}}{\partial t_{a} \partial t_{b} \partial t_{c}}-\frac{\partial^{3}}{\partial t_{a} \partial \bar{t}_{b} \partial \bar{t}_{c}}\right),
\end{gathered}
$$

and two types of time-variables, denoted through $t_{k}$ and $\bar{t}_{k}$. are ossociated with two orthogonal directions in the Cartan plane of $A_{2}: \mathbf{e}=\frac{\boldsymbol{\alpha}_{1}}{\sqrt{2}}, \overline{\mathbf{e}}=\frac{\sqrt{3} \boldsymbol{\nu}_{2}}{\sqrt{2}}$. 因

All other formulas, however, are very simple: Conformal model is usually that of the $r$ free fields, $S \sim \int \bar{\partial} \phi \partial \phi d^{2} z$, which is used to describe representation of the level one Kac-Moody algebra, associated with $\mathcal{A}$. Hamiltonian

$$
H\left(t^{(1)} \ldots t^{(r+1)}\right)=\sum_{\lambda=1}^{r+1} \sum_{k>0} t_{k}^{(\lambda)} \boldsymbol{\mu}_{\lambda} \boldsymbol{J}_{k}
$$

where $\left\{\boldsymbol{\mu}_{\lambda}\right\}$ are associated with "fundamental weight" vectors $\boldsymbol{\nu}_{\lambda}$ in Cartan hyperplane and in the simplest case of $\mathcal{A}=A_{r}(S L(r+1))$ satisfy

$$
\boldsymbol{\mu}_{\lambda} \cdot \boldsymbol{\mu}_{\lambda^{\prime}}=\delta_{\lambda \lambda^{\prime}}-\frac{1}{r+1}, \quad \sum_{\lambda=1}^{r+1} \boldsymbol{\mu}_{\lambda}=0
$$

thus only $r$ of the time variables $t^{(1)} \ldots t^{(r+1)}$ are linearly independent. Relation between differential operators $W_{n}^{(a)}(t)$ and operators $\mathrm{W}_{n}^{(a)}$ in the CFT is now defined by

$$
\begin{array}{r}
W_{n}^{(a)}\langle\boldsymbol{N}| e^{H(t)} \ldots=\langle\boldsymbol{N}| e^{H(t)} \mathrm{W}_{i}^{(a)} \ldots, \\
a=2, \ldots, p ; \quad i \geq 1-a,
\end{array}
$$

where

$$
\begin{array}{r}
\mathrm{W}_{n}^{(a)}=\oint z^{a+n-1} \mathrm{~W}^{(a)}(z) \\
\mathrm{W}^{(a)}(z)=\sum_{\lambda}\left[\boldsymbol{\mu}_{\lambda} \partial \phi(z)\right]^{a}+\ldots
\end{array}
$$

\footnotetext{
${ }^{9}$ Such orthogonal basis is especially convenient for discussion of integrability properties of the model, these $t$ and $\bar{t}$ are linear combinations of time-variables $t_{k}^{\lambda}$ appearing in eqs. (2.27) and (2.32).
} 
are spin- $a$ generators of the $\mathbf{W}_{r+1}^{\mathcal{A}}$ algebra. The screening charges, that commute with all the $\mathrm{W}^{(a)}(z)$ are given by

$$
Q^{(\alpha)}=\oint J^{(\alpha)}=\oint e^{\alpha \phi}
$$

$\{\boldsymbol{\alpha}\}$ being roots of finite-dimensional simply laced Lie algebra $\mathcal{A}$.

Thus partition function arises in the form:

$$
Z_{\boldsymbol{N}}^{\mathcal{A}}\{t\}=\left\langle\boldsymbol{N}\left|e^{H(t} G\left\{Q^{(\alpha)}\right\}\right| 0\right\rangle
$$

where $G$ is an exponential function of screening charges. Evaluation of the free-feild correlator gives:

$$
\begin{aligned}
Z_{\boldsymbol{N}}^{\mathcal{A}}\{t\} \sim \int \prod_{\alpha} & {\left[\prod_{i=1}^{N_{\alpha}} d z_{i}^{(\alpha)} \exp \left(\sum_{\lambda ; k>0} t_{k}^{(\lambda)}\left(\boldsymbol{\mu}_{\lambda} \boldsymbol{\alpha}\right)\left(z_{i}^{(\alpha)}\right)^{k}\right)\right] \times } \\
& \times \prod_{(\alpha, \beta)} \prod_{i=1}^{N_{\alpha}} \prod_{j=1}^{N_{\beta}}\left(z_{i}^{(\alpha)}-z_{j}^{(\beta)}\right) \boldsymbol{\alpha} \boldsymbol{\beta}
\end{aligned}
$$

In fact this expression can be rewritten in terms of an $r$-matrix integral - a "conformal multimatrix model":

$$
\begin{array}{r}
Z_{\boldsymbol{N}}^{\mathcal{A}}\left\{t^{(\alpha)}\right\}=c_{N}^{p-1} \int_{N \times N} d H^{(1)} \ldots d H^{(p-1)} \prod_{\alpha=1}^{p-1} e^{\sum_{k=0}^{\infty} t_{k}^{(\alpha)} \operatorname{Tr} H_{(\alpha)}^{k}} . \\
\cdot \prod_{(\alpha, \beta)} \operatorname{Det}\left(H^{(\alpha)} \otimes I-I \otimes H^{(\alpha+1)}\right)^{\boldsymbol{\alpha} \boldsymbol{\beta}}
\end{array}
$$

In the simplest case of $\mathbf{W}_{3}$ algebra eq.(2.32) with insertion of only two (of the six) screenings $Q_{\alpha_{1}}$ and $Q_{\alpha_{2}}$ turns into

$$
\begin{gathered}
Z_{N_{1}, N_{2}}^{A_{2}}(t, \bar{t})=\frac{1}{N_{1} ! N_{2} !}\left\langle N_{1}, N_{2}\left|e^{H(t, \bar{t})}\left(Q^{\left(\alpha_{1}\right)}\right)^{N_{1}}\left(Q^{\left(\alpha_{2}\right)}\right)^{N_{2}}\right| 0\right\rangle= \\
=\frac{1}{N_{1} ! N_{2} !} \prod_{i} \int d x_{i} e^{U\left(x_{i}\right)} \prod_{j} \int d y_{j} e^{\bar{U}\left(y_{i}\right)} \Delta(x) \Delta(x, y) \Delta(y),
\end{gathered}
$$

where $\Delta(x, y) \equiv \Delta(x) \Delta(y) \prod_{i, j}\left(x_{i}-y_{j}\right)$. This model is associated with the algebra $\mathcal{A}=A_{2}(S L(3))$, while the original 1-matrix model (2.21)-(2.23) with $\mathcal{A}=A_{1}(S L(2))$. 
The whole series of models $\left(2.32\right.$ 2.33) for $\mathcal{A}=A_{r}(S L(r+1))$ is distinguished by its relation to the level $k=1$ simply-laced Kac-Moody algebras. In this particular situation the underlying conformal model has integer central charge $c=r=\operatorname{rank} \mathcal{A}$ and can be "fermionized". TU The main feature of this formulation is that the Kac-Moody currents (which after integration turn into "screening charges" in the above construction) are quadratic in fermionic fields, while they are represented by exponents in the free-boson formulation.

In fact fermionic (spinor) model naturally possesses $G L(r+1)$ rather than $S L(r+1)$ symmetry (other simply-laced algebras can be embedded into larger $G L$-algebras and this provides fermionic descriprion for them in the case of $k=1$ ). The model contains $r+1$ spin- $1 / 2$ fields $\psi_{i}$ and their conjugate $\tilde{\psi}_{i}$ (b,c-systems);

$$
S=\sum_{j=1}^{r+1} \int \tilde{\psi}_{j} \bar{\partial} \psi_{j} d^{2} z,
$$

central charge $c=r+1$, and operator algebra is

$$
\begin{aligned}
\tilde{\psi}_{j}(z) \psi_{k}\left(z^{\prime}\right) & =\frac{\delta_{j k}}{z-z^{\prime}}+: \tilde{\psi}_{j}(z) \psi_{k}\left(z^{\prime}\right): \\
\psi_{j}(z) \psi_{k}\left(z^{\prime}\right) & =\left(z-z^{\prime}\right) \delta_{j k}: \psi_{j}(z) \psi_{k}\left(z^{\prime}\right):+\left(1-\delta_{j k}\right): \psi_{j}(z) \psi_{k}\left(z^{\prime}\right): \\
\tilde{\psi}_{j}(z) \tilde{\psi}_{k}\left(z^{\prime}\right) & =\left(z-z^{\prime}\right) \delta_{j k}: \tilde{\psi}_{j}(z) \tilde{\psi}_{k}\left(z^{\prime}\right):+\left(1-\delta_{j k}\right): \tilde{\psi}_{j}(z) \tilde{\psi}_{k}\left(z^{\prime}\right):
\end{aligned}
$$

The Kac-Moody currents of level $k=1 G L(r+1)$ are just $J_{j k}=: \tilde{\psi}_{j} \psi_{k}$ : $j, k=1 \ldots r+1$, and screening charges are $Q^{(\alpha)}=i E_{j k}^{(\alpha)} \oint: \tilde{\psi}_{j} \psi_{k}:$, where $E_{j k}^{(\alpha)}$ are representatives of the roots $\boldsymbol{\alpha}$ in the matrix representation of $G L(r+1)$. Cartan subalgebra is represented by $J_{j j}$, while positive and negative Borel subalgebras - by $J_{j k}$ with $j<k$ and $j>k$ respectively. In eq.(2.23) $Q_{+}=$ $i \oint \tilde{\psi}_{1} \psi_{2}, \quad Q_{-}=i \oint \tilde{\psi}_{2} \psi_{1} \quad$ while in eq. 2.34$) Q^{\left(\alpha_{1}\right)}=i \oint \tilde{\psi}_{1} \psi_{2}, \quad Q^{\left(\alpha_{2}\right)}=$ $i \oint \tilde{\psi}_{1} \psi_{3} \quad\left(\right.$ and $Q^{\left(\alpha_{3}\right)}=i \oint \tilde{\psi}_{2} \psi_{3}, \quad Q^{\left(\alpha_{4}\right)}=i \oint \psi_{2} \psi_{1}, \quad Q^{\left(\alpha_{5}\right)}=i \oint \tilde{\psi}_{3} \psi_{1}, \quad Q^{\left(\alpha_{6}\right)}=$ $\left.i \oint \tilde{\psi}_{3} \psi_{2}\right)$. $Q^{\left(\alpha_{6}\right)}$ can be substituted instead of $Q^{\left(\alpha_{2}\right)}$ in (2.34) without changing

\footnotetext{
10 This is possible only for very special Kac-Moody algebras, and such formulation is important in order to deal with conventional formulation of integrability, which usually involves commuting Hamiltonian flows (not just a closed algebra of flows) and fermionic realization of the universal module space (universal Grassmannian). In fact these restrictions are quite arbitrary and can be removed (though this is not yet done in full details), see section 4 below for more detailed discussion.
} 
the answer. For generic $r$ the similar choice of "adjacent" (not simple!) roots (such that their scalar products are +1 or 0 ) leads to selection of the following $r$ screening operators $Q^{(1)}=i \oint \tilde{\psi}_{1} \psi_{2} \quad Q^{(2)}=-i \oint \psi_{2} \tilde{\psi}_{3}, Q^{(3)}=i \oint \tilde{\psi}_{3} \psi_{4}, \ldots$, i.e. $Q^{(j)}=i \oint \tilde{\psi}_{j} \psi_{j+1}$ for odd $j$ and $Q^{(j)}=-i \oint \psi_{j} \tilde{\psi}_{j+1}$ for even $j$.

\subsection{Gross-Newman equation}

We turn now to consideration of the WI for another sort of matrix models. This subject concerns at least two important classes: the conventional discrete two-matrix models and Kontsevich models. As it was explained in the Introduction the theories of the second type arise in consderation of the $(p, 1)$ continuous matrix models, as well as in the study of topological Landau-Ginzburg theories, while the two-matrix model is believed to exhibit a rich pattern of continuous limits and is capable to provide representatives of all the $(p, q)$ universality classes (this line of reasoning, however, has never been really developed and we shall not discuss it in these notes).

The starting point and the basic example is provided by the integral

$$
\mathcal{F}_{V, n}\{L\} \equiv \int_{n \times n} d X e^{-\operatorname{tr} V(X)+\operatorname{tr} L X}
$$

over $n \times n$ Hermitean matrix, which we shall further refer to as "Kontsevich integral", keeping in mind its most important application (though this obvious quantity has been of course considered by many other people). It may seem that the action in this integral is not of the most general type and we can no longer perform arbitrary change of variables $X \longrightarrow f(X)$ without changing the functional form of the integral. In fact this is incorrect, because "external field" $L$ is matrix valued and coupled to $X$ linearly, and therefore any correlator of $X$-fields can be represented through $L$-derivatives. Consider again the shift $X \rightarrow X+\epsilon_{n} X^{n+1}, n \geq-1$. Invariance of the integral implies:

$$
\int d X e^{-\operatorname{tr} V(X)+\operatorname{tr} L X} \operatorname{tr} \epsilon_{n}\left(-X^{n+1} V^{\prime}(X)+L X^{n+1}+\sum_{k=0}^{n} X^{k} \operatorname{tr} X^{n-k}\right)=0,
$$

which can be rewritten as ${ }^{11}$

$$
\operatorname{tr} \epsilon_{n}\left(\left(-\frac{\partial}{\partial L_{\mathrm{tr}}}\right)^{n+1} V^{\prime}\left(\frac{\partial}{\partial L_{\mathrm{tr}}}\right)+L\left(-\frac{\partial}{\partial L_{\mathrm{tr}}}\right)^{n+1}+\right.
$$

\footnotetext{
${ }^{11}$ The obvious relation is used here: $X_{\gamma \delta} e^{\operatorname{tr} L X}=\frac{\partial}{L_{\delta \gamma}} e^{\operatorname{tr} L X}$. Note that the order of
} 


$$
\begin{aligned}
& \left.\sum_{k=0}^{n}\left(-\frac{\partial}{\partial L_{\mathrm{tr}}}\right)^{k} \operatorname{tr}\left(-\frac{\partial}{\partial L_{\mathrm{tr}}}\right)^{n-k}\right) \mathcal{F}_{V}\{L\}= \\
& =\operatorname{tr} \epsilon_{n}\left(-\frac{\partial}{\partial L_{\mathrm{tr}}}\right)^{n+1}\left(V^{\prime}\left(\frac{\partial}{\partial L_{\mathrm{tr}}}\right)-L\right) \mathcal{F}_{V}\{L\}=0
\end{aligned}
$$

This system is in fact equivalent to a single matrix-valued equation

$$
\left(V^{\prime}\left(\frac{\partial}{\partial L_{\text {tr }}}\right)-L\right) \mathcal{F}_{V}\{L\}=0 .
$$

As well as I know this equation was first written down in [54, therefore it will be refered to as the Gross-Newman (GN) equation. It was rediscovered and implications for the theory of matrix models were investigated in [24], [30], [38].

There are essentially two types of corollaries, which will be discussed in the next two subsections. First, GN equation can be used to characterize the function $\mathcal{F}_{V}\{L\}$ itself. This will lead us to consideration of Kontsevich models. Second, it can be used to derive equations for the 2-matrix model, which arises after $\mathcal{F}_{V}\{L\}$ is further integrated with some weight over $L$.

\subsection{Ward identities for Generalized Kontsevich Model}

Being just the complete set of equation of motion the GN equation (2.37) provides complete information about the function $\mathcal{F}_{V}\{L\}$. However, this statement needs to be formulated more carefully. A need for this comes, for example, from the observation that operators

$$
\operatorname{tr} L^{m}\left(V^{\prime}\left(\frac{\partial}{\partial L_{\mathrm{tr}}}\right)-L\right)
$$

do not form a closed algebra: their commutators have some different functional form. One of the reasons for these complications is that eq.(2.37) does not account explicitly for a very important property of $\mathcal{F}_{V}\{L\}$ : this function in fact depends only on the eigenvalues of $L$. This information should be still added somehow to the GN equation. We shall analyze this issue of eigenvalue-dependence in more details in next sections. For our current purposes this argument implies that one should try to express equation (2.37) in

matrix indices $\gamma \delta$ is reversed at the r.h.s. as compared to the l.h.s., i.e. derivatives are in fact w.r.to transponed matrix $L: \quad f(X) e^{\operatorname{tr} L X}=f\left(\frac{\partial}{\partial L_{t r}}\right) e^{\operatorname{tr} L X}$ (at least for any function $f(x)$, which can be represented as a formal seria in integer powers of $X$ ). 
terms of eigenvalues. Here, however, one should be carefull again. Clearly, not only $\mathcal{F}_{V}\{L\}$ depends on eigenvalues, it depends on their "symmetric" (Weyl-group invariant) combinations, i.e. it rather depends on quantities like $\operatorname{tr} L^{a}$ then on particular eigenvalues. Moreover, powers $a$ here should be negative and fractional.

Indeed, integrals like (2.35) are usually understood as analytical continuation from some values of parameters in the potential $V$, when integral is convergent. They can be also related to the formal (perturbation) seria arising when integrand is expanded around a stationary point. To begin with it is reasonable to take $n=1$ i.e. consider just an ordinary integral. For the sake of simplicity also take particular $V(x)=-\frac{x^{p+1}}{p+1}$. Then the stationary point is at $x=\lambda^{\frac{1}{p}}$ and

$$
\int d x e^{-\frac{x^{p+1}}{p+1}+l x} \sim l^{-\frac{p-1}{2}} e^{\frac{p}{p+1} l^{\frac{p+1}{p}}} \sum_{k \geq 0} c_{k} l^{-\frac{k}{p}} .
$$

It is now easy to understand what should be done in the general situation with matrices and arbitrary potentials. First of all, one needs to solve equation for the stationary point, $V^{\prime}(X)=L$. For this purpose it is most convenient to introduce a new matrix variable $\Lambda$ instead of $L$, which by definition satisfies $V^{\prime}(\Lambda)=L$. Then stationary point is just $X=\Lambda$. Second, one should separate the analogue of the complicated prefactor (quasiclassical contribution):

$$
\mathcal{C}_{V}\{\Lambda\}=(2 \pi)^{n^{2} / 2} \frac{e^{\operatorname{tr}\left(\Lambda V^{\prime}(\Lambda)-V(\Lambda)\right)}}{\sqrt{\operatorname{det} V^{\prime \prime}(\Lambda)}},
$$

Then the function that describes pure "quantum" contribution एా

$$
\mathcal{Z}_{V}\{T\} \equiv \mathcal{C}_{V}\{\Lambda\}^{-1} \mathcal{F}_{V}\left\{V^{\prime}(\Lambda)\right\}
$$

\footnotetext{
${ }^{12}$ The "classical action" in (2.40) can be also represented as $\operatorname{tr}\left(\Lambda V^{\prime}(\Lambda)-V(\Lambda)\right)=$ $\operatorname{tr} \int \Lambda d V^{\prime}(\Lambda)$. Determinant of quadratic fluctuations is defined as

$$
(2 \pi)^{n^{2} / 2}\left(\operatorname{det} V^{\prime \prime}(\Lambda)\right)^{-1 / 2} \sim \int d Y e^{-\operatorname{tr} V_{2}(\Lambda, Y)},
$$

where $V_{2}(\Lambda, Y) \equiv \lim _{\epsilon \rightarrow 0} \frac{1}{\epsilon^{2}}\left(V(\Lambda+\epsilon Y)-V(\Lambda)-\epsilon V^{\prime}(\Lambda) Y\right)$. For $V(\Lambda)=\frac{\Lambda^{p+1}}{p+1}$ we have $V^{\prime \prime}(\Lambda)=\left(\sum_{k=0}^{p-1} \Lambda^{k} \otimes \Lambda^{p-k-1}\right)$. One could easily choose an "opposite" parametrization in eq.2.42): $T_{k}=-\frac{1}{k} \operatorname{tr} \Lambda^{-k}$. Though not quite obvious, this never influences any results (see section 2.10 for an example). Our choice of signs is motivated by simplification of
} 
to be refered as partition function of the Generalized Kontsevich Model (GKM) [30], can be represented as a formal (perturbation) series expansion in variables

$$
T_{k}=\frac{1}{k} \operatorname{tr} \Lambda^{-k}
$$

GN (2.37) equation can be now rewritten as a set of differential equations for $\mathcal{Z}_{V}\{T\}$. Indeed, we already have:

$$
\mathcal{C}_{V}^{-1}\left(V^{\prime}\left(\frac{\partial}{\partial L_{\text {tr }}}\right)-L\right) \mathcal{C}_{V} \mathcal{Z}_{V}\{T\}=0,
$$

but it is still necessary to express the operator at the l.h.s. in terms of $T$. This is in fact possible to do, using the relation:

$$
\frac{\partial}{\partial L_{t r}} \mathcal{Z}_{V}\{T\}=\sum_{k} \frac{\partial T_{k}}{\partial L_{t r}} \frac{\partial Z}{\partial T_{k}}
$$

and substituting the traces of $\Lambda$-matrices, which can arise in the process of calculation, by $T$ 's. It is important only that $\Lambda$ 's usually appear in negative powers: this is already achieved by the choice of a proper normalization factor $\mathcal{C}_{V}\{\Lambda\}$. For monomial potential $V_{p}(X)=\frac{X^{p+1}}{p+1}$ this is especialy simple: $L=\Lambda^{p}$ and $\frac{\partial T_{k}}{\partial L_{t r}}=-\frac{1}{p} \Lambda^{-p-k}$.

This reasoning allows one to rewrite eq. 2.43) identically in the form

$$
\sum_{l} \Lambda^{-l} \mathcal{O}_{l}(T) \mathcal{Z}_{V}\{T\}=0
$$

where $\mathcal{O}_{l}$ are some differential operators, depending on the shape of $V$, but independent on the size $n$ of the matrix (as all the above reasoning never refered to particular values of $n$, except for a sample example at the very beginning). It remains to use the fact that matrix $L$ can be arbitrary large and have arbitrarily many independent entries, in order to conclude that we derived a set of constraints on $\mathcal{Z}_{V}$ in the form

$$
\mathcal{O}_{l}(T) \mathcal{Z}_{V}\{T\}=0
$$

formulas for the GKM, including the relation between $L$ and $\Lambda$. Instead, some sign factors appear in formulas, related to Toda-like representations of partition functions and those involving $\tilde{W}$-operators. 
For potential $V$ of degree $p+1$ these appear to be exactly the "continuous Virasoro constraints". See refs. 24] and 30 for detailed analysis of the Virasoro case $p=2$ (associated with the pure topological gravity and with the double-scaling limit of the 1-matrix model), and 55 for the exhaustive presentation of the case of $p=3$.

\subsection{Discrete Virasoro constraints for the Gaussian Kont- sevich model}

As a simplest illustration of the technique, described in the previous subsection, we derive now the constraints for the Gaussian Kontsevich model [56] with potential $V(X)=\frac{1}{2} X^{2}$ :

$$
\mathcal{Z}_{\frac{X^{2}}{2}}\{N, T\}=\frac{e^{-\operatorname{tr} \frac{L^{2}}{2}}}{(\operatorname{det} L)^{N}} \int d X(\operatorname{det} X)^{N} e^{-\operatorname{tr} \frac{X^{2}}{2}+L X} .
$$

In this case $L=V^{\prime}(\Lambda)=\Lambda$, and the time-variables are just

$$
T_{k}=\frac{1}{k} \operatorname{tr} \Lambda^{-k}=\frac{1}{k} \operatorname{tr} L^{-k} .
$$

To make the model non-trivial an extra "zero-time" variable $N$ [36] is introduced, which was not included into the previous definition (2.41). Now note that the $N$-dependence of Kontsevich integral (2.35) can be described simply as an extra term in the potential: $V(X) \rightarrow \hat{V}(X)=V(X)-N \log X$ (though this can not be done neither in the quasiclassical factor $\mathcal{C}_{V}$ nor in the definition of time-variables $T$ ). Since the GN equation depends only on Kontsevich equation, we can use it with $V$ substituted by $\hat{V}$. Then we have instead of (2.43):

$$
\begin{gathered}
\frac{e^{-\operatorname{tr} \frac{L^{2}}{2}}}{(\operatorname{det} L)^{N}}\left(\frac{\partial}{\partial L_{t r}}\right)^{n+1} \cdot\left(\frac{\partial}{\partial L_{t r}}-N\left(\frac{\partial}{\partial L_{t r}}\right)^{-1}-L\right) . \\
\cdot(\operatorname{det} L)^{N} e^{+\operatorname{tr} \frac{L^{2}}{2}} \mathcal{Z}_{\frac{X^{2}}{2}}\{N, T\}=0 .
\end{gathered}
$$

In order to get rid of the integral operator $\left(\frac{\partial}{\partial L}\right)^{-1}$ one should take here $n \geq 0$ rather than $n \geq-1$. In fact all the equations with $n>0$ follow from the one 
with $n=0$, and we restrict our consideration to the last one. For $n=0$ we obtain from (2.49):

$$
\left(\left(\frac{\partial}{\partial L_{t r}}+\frac{N}{L}+L\right)^{2}-2 N-L\left(\frac{\partial}{\partial L_{t r}}+\frac{N}{L}+L\right)\right) \mathcal{Z}=0
$$

or

$$
\left(\left(\frac{\partial}{\partial L_{t r}}\right)^{2}+\left(L+\frac{2 N}{L}\right) \frac{\partial}{\partial L_{t r}}+\frac{N^{2}}{L^{2}}-\frac{N}{L} \operatorname{tr} \frac{1}{L}\right) \mathcal{Z}=0,
$$

and it remains to substitute:

$$
\begin{aligned}
\frac{\partial \mathcal{Z}}{\partial L_{t r}} & =-\sum_{k=0}^{\infty} \frac{1}{L^{k+1}} \frac{\partial \mathcal{Z}}{\partial T_{k}} \\
\frac{\partial^{2} \mathcal{Z}}{\partial L_{t r}^{2}} & =\sum_{k=1}^{\infty}\left(\sum_{a=1}^{k+1} \frac{1}{L^{k+2-a}} \operatorname{tr} \frac{1}{L^{a}}\right) \frac{\partial \mathcal{Z}}{\partial T_{k}}+\sum_{k, l=1}^{\infty} \frac{1}{L^{k+l+2}} \frac{\partial^{2} \mathcal{Z}}{\partial T_{k} \partial T_{l}}= \\
& =\sum_{m=-1}^{\infty} \frac{1}{L^{m+2}}\left(\sum_{k>\max (m, 0)}\left(\operatorname{tr} \frac{1}{L^{k-m}}\right) \frac{\partial \mathcal{Z}}{\partial T_{k}}+\sum_{k=1}^{m-1} \frac{\partial^{2} \mathcal{Z}}{\partial T_{k} \partial T_{m-k}}\right)
\end{aligned}
$$

and finally obtain:

$$
\begin{aligned}
\sum_{m=-1}^{\infty} & \frac{1}{L^{m+2}}\left(\sum_{k=1+\delta_{m,-1}}^{\infty}\left(\operatorname{tr} \frac{1}{L^{k}}\right) \frac{\partial}{\partial T_{k+m}}+\sum_{k=1}^{m-1} \frac{\partial^{2}}{\partial T_{k} \partial T_{m+k}}-\right. \\
& \left.-\frac{\partial}{\partial T_{m+2}}-2 N \frac{\partial}{\partial T_{m}}+N^{2} \delta_{m, 0}-N\left(\operatorname{tr} \frac{1}{L}\right) \delta_{m,-1}\right) \mathcal{Z}= \\
& =\sum_{m=-1}^{\infty} \frac{1}{L^{m+2}} e^{N T_{0}} L_{m}(T+r) e^{-N T_{0}} \mathcal{Z}=0 .
\end{aligned}
$$

Here $L_{m}(t)$ are just the generators (2.7) of the discrete Virasoro algebra (2.6):

$$
e^{N t_{0}} L_{m}(t) e^{-N t_{0}}=e^{N t_{0}}\left(\sum_{k=1}^{\infty} k t_{k} \frac{\partial}{\partial t_{k+m}}+\sum_{k=0}^{m} \frac{\partial^{2}}{\partial t_{k} \partial t_{m-k}}\right) e^{-N t_{0}} .
$$

and at the r.h.s. of (2.51) $r_{k}=-\frac{1}{2} \delta_{k, 2} \cdot$ [3

13 This small correction is a manifestation of a very general phenomenon: from the point of view of symmetries (Ward identities) it is more natural to consider $Z_{V}$ not as a function of $T$-variables, but of some more complicated combination $\hat{T}_{k}+r_{k}$, depending on the shape of potential $V$. If $V$ is a polinomial of degree $p+1, \hat{T}_{k}=\frac{1}{k} \operatorname{tr}\left(V^{\prime}(\lambda)\right)^{-k / p}$, while $r_{k}=\frac{p}{k(p-k)} \operatorname{Res}\left(V^{\prime}(\mu)\right)^{1-\frac{k}{p}} d \mu$. For monomial potentials these expressions become very simple: $\hat{T}_{k}=T_{k}$ and $r_{k}=-\frac{p}{p+1} \delta_{k, p+1}$. See [39] and section 4.9 below for more details. In most places in these notes we prefer to use invariant potential-independent times $T_{k}$, instead of $\hat{T}_{k}$, but then Ward identites acquire some extra terms with $r_{k}$ (which in fact will be very simple in our examples, which are all given for monomial potentials). 
Thus we found that the WI of the Gaussian Kontsevich model (2.47) coincide with those of the ordinary 1-matrix model, moreover the size of the matrix $N$ in the latter model is associated with the "zero-time" in the former one. This result [56] of course implies, that the two models are identical:

$$
e^{-N T_{0}} \mathcal{Z}_{\frac{X^{2}}{2}}\left\{N, T_{1}, T_{2}, \ldots\right\} \sim Z_{N}\left\{T_{0}, T_{1}, T_{2}, \ldots\right\}
$$

We shall discuss direct connection between these two matrix integrals (2.1) and (2.47) in the next section, after some more details will be presented about the structure of "eigenvalue" matrix models.

\subsection{Continuous Virasoro constraints for the $V=\frac{X^{3}}{3}$ Kontsevich model}

This example is a little more complicated than that in the previous subsection, and we do not present calculations in full details (see [24 and [30]). Our goal is to demonstrate that the constraints which arise in this model, though still form (Borel subalgebra of) some Virasoro algebra, are different from (2.6). From the point of view of the CFT-formulation the relevant model is that of the twisted (in this particular case - antiperiodic) free fields. These so called "continuous Virasoro constraints" give the simplest illustration of the difference between discrete and continuous matrix models: this is essentially the difference between "homogeneous" (Kac-Frenkel) and "principal" (soliton vertex operator) representation of the level $k=1$ Kac-Moody algebra. From the point of view of integrable hierarchies this is the difference between Toda-chain-like and KP-like hierarchies. We shall come back to a more detailed discussion of this difference later, when the "multi-scaling continuum limit" will be considered.

Another (historical) aspect of the same relation also deserves mentioning, since it also illustrates the interrelation between different models. The discrete 1-matrix model arises naturally in description of quantum $2 d$ gravity as sum over 2-geometries in the formalism of random equilateral triangulations. The model, however, decribes only lattice approximation to $2 d$ gravity and (double-scaling) coninuum limit should be taken in order to obtain the real (continuous) theory of $2 d$ gravity. This limit was originally formulated in terms of the contraint algebra (equations of motion or "loop" or "SchwingerDyson" equations - terminology is taste-dependent), leaving open the prob- 
lem of what is the form of partition function $\mathcal{Z}^{\text {cont }}\{T\}$ of continuous theory. Since the relevant algebra appeared to be just the WI for Kontsevich model (with $V(X)=\frac{X^{3}}{3}$ ), this proves that the latter one is exactly the continuous theory of pure $2 d$ gravity. At the same time, Kontsevich model itself can be naturally introduced as a theory of topological gravity (in fact this is how the model was originally discovered in [22]). From this point of view the constraint algebra, to be discussed below in this subsection, plays central role in the proof of equivalence between pure $2 d$ quantum gravity and pure topological gravity (in both cases "pure" means that "matter" fields are not introduced).

After these introductory remarks we proceed to calculations. Actually they just repeat those for the Gaussian model, performed in the previous subsection, though formulas get somewhat more complicated. This time we do not include zero-time $N$ and just use eq.(2.37) with $V(X)=\frac{X^{3}}{3}$. Now it is also much more tricky (though possible) to work in matrix notations (because fractional powers of $L$ will be involved) and we rewrite everything in terms of the eigenvalues of $L$.

We substitute

$$
\begin{gathered}
\mathcal{C}_{\frac{X^{3}}{3}}=\frac{\prod_{\delta} e^{\frac{2}{3} \lambda_{\delta}^{3 / 2}}}{\sqrt{\prod_{\gamma, \delta}\left(\sqrt{\lambda_{\delta}}+\sqrt{\lambda_{\gamma}}\right)}} \\
\left(\frac{\partial^{2}}{\partial L_{t r}^{2}}\right)_{\gamma \gamma}=\frac{\partial^{2}}{\partial \lambda_{\gamma}^{2}}+\sum_{\delta \neq \gamma} \frac{1}{\lambda_{\gamma}-\lambda_{\delta}}\left(\frac{\partial}{\partial \lambda_{\gamma}}-\frac{\partial}{\partial \lambda_{\delta}}\right)
\end{gathered}
$$

and introduce a special notation for

$$
\frac{\mathcal{D}}{\mathcal{D} \lambda_{\gamma}} \equiv \mathcal{C}_{\frac{X^{3}}{3}}^{-1} \frac{\partial}{\partial \lambda_{\gamma}} \mathcal{C}_{\frac{X^{3}}{3}}=\frac{\partial}{\partial \lambda_{\gamma}}+\sqrt{\lambda_{\gamma}}-\frac{1}{4 \lambda_{\gamma}}-\frac{1}{2} \sum_{\delta \neq \gamma} \frac{1}{\sqrt{\lambda_{\gamma}}\left(\sqrt{\lambda_{\delta}}+\sqrt{\lambda_{\gamma}}\right)}
$$

Then (2.37) turns into

$$
\left(\left(\frac{\mathcal{D}}{\mathcal{D} \lambda_{\gamma}}\right)^{2}+\sum_{\delta \neq \gamma} \frac{1}{\lambda_{\gamma}-\lambda_{\delta}}\left(\frac{\mathcal{D}}{\mathcal{D} \lambda_{\gamma}}-\frac{\mathcal{D}}{\mathcal{D} \lambda_{\delta}}\right)\right) \mathcal{Z}_{\frac{X^{3}}{3}}\{T\}=0 .
$$

Now we need explicit expression for $T$ :

$$
T_{k}=\frac{1}{k} L^{-k}
$$


and as we already know from the previous subsection we also need

$$
r_{k}=-\frac{2}{3} \delta_{k, 3} .
$$

It will not be explained untill we turn to consideration of integrable structure of Kontsevich model in the following sections, but $\mathcal{Z}_{\frac{X^{3}}{3}}\{T\}$ is in fact independent of all time-variables with even numbers (subscripts). Therefore we can take only $k=2 a+1$ in (2.55),

$$
\begin{aligned}
T_{2 a+1} & =\frac{1}{2 a+1} \sum_{\delta} \lambda_{\delta}^{-a-\frac{1}{2}}, \\
r_{2 a+1} & =-\frac{2}{3} \delta_{a, 1}
\end{aligned}
$$

and

$$
\begin{aligned}
& \frac{\partial}{\partial \lambda_{\gamma}} \mathcal{Z}_{\frac{X^{3}}{3}}\{T\}=\sum_{a=0}^{\infty} \frac{\partial T_{2 a+1}}{\partial \lambda_{\gamma}} \frac{\partial \mathcal{Z}}{\partial T_{2 a+1}}=-\frac{1}{2} \sum_{a=0}^{\infty} \lambda_{\gamma}^{-a-\frac{3}{2}} \frac{\partial \mathcal{Z}}{\partial T_{2 a+1}} \\
& \frac{\partial^{2}}{\partial \lambda_{\gamma}^{2}} \mathcal{Z}_{\frac{X^{3}}{3}}\{T\}=\frac{1}{4} \sum_{a, b=0}^{\infty} \lambda_{\gamma}^{-a-b-3} \frac{\partial \mathcal{Z}}{\partial T_{2 a+1} \partial T_{2 b+1}}+\frac{1}{2} \sum_{a=0}^{\infty}\left(a+\frac{3}{2}\right) \lambda_{\gamma}^{-a-\frac{5}{2}} \frac{\partial \mathcal{Z}}{\partial T_{2 a+1}} .
\end{aligned}
$$

These expressions should be now substituted into (2.54) and we obtain:

$$
\begin{gathered}
\frac{1}{4} \sum_{a, b=0}^{\infty} \lambda_{\gamma}^{-a-b-3} \frac{\partial \mathcal{Z}}{\partial T_{2 a+1} \partial T_{2 b+1}}+ \\
+\sum_{a=0}^{\infty}\left[\frac{1}{2} \sum_{a=0}^{\infty}\left(a+\frac{3}{2}\right) \lambda_{\gamma}^{-a-\frac{5}{2}}-\frac{1}{2} \sum_{\delta \neq \gamma} \frac{1}{\lambda_{\gamma}-\lambda_{\delta}}\left(\lambda_{\gamma}^{-a-\frac{3}{2}}-\lambda_{\delta}^{-a-\frac{3}{2}}\right)-\right. \\
\left.-\left(\sqrt{\lambda_{\gamma}}-\frac{1}{4 \lambda_{\gamma}}-\frac{1}{2} \sum_{\delta \neq \gamma} \frac{1}{\sqrt{\lambda_{\gamma}}\left(\sqrt{\lambda_{\delta}}+\sqrt{\lambda_{\gamma}}\right)}\right) \lambda_{\gamma}^{-a-\frac{3}{2}}\right] \frac{\partial \mathcal{Z}}{\partial T_{2 a+1}}+ \\
+[\ldots] \mathcal{Z}=\sum_{n=-1}^{\infty} \frac{1}{\lambda_{\gamma}^{n+2}} \mathcal{L}_{n} \mathcal{Z}
\end{gathered}
$$


with

$$
\begin{gathered}
\mathcal{L}_{2 n}=\sum_{a=0}^{\infty}\left(a+\frac{1}{2}\right)\left(T_{2 a+1}+r_{2 a+1}\right) \times \\
\times \frac{\partial}{\partial T_{2 a+2 n+1}}+\frac{1}{4} \sum_{\substack{a+b=n-1 \\
a, b \geq 0}} \frac{\partial^{2}}{\partial T_{2 a+1} \partial T_{2 b+1}}+\frac{1}{16} \delta_{n, 0}+\frac{1}{4} T_{1}^{2} \delta_{n,-1}= \\
=\frac{1}{2} \sum_{\text {odd } k=1}^{\infty} k\left(T_{k}+r_{k}\right) \frac{\partial}{\partial T_{k+2 n}}+\frac{1}{4} \sum_{\text {odd } k=1}^{2 n-1} \frac{\partial^{2}}{\partial T_{k} \partial T_{2 n-k}}+\frac{1}{16} \delta_{n, 0}+\frac{1}{4} T_{1}^{2} \delta_{n,-1} .
\end{gathered}
$$

Factor $\frac{1}{2}$ in front of the first term at the r.h.s. in (2.59) is important for $\mathcal{L}_{2 n}$ to satisfy the properly normalized Virasoro algebra: ${ }^{\text {th }}$

$$
\left[\mathcal{L}_{2 n}, \mathcal{L}_{2 m}\right]=(n-m) \mathcal{L}_{2 n+2 m} .
$$

Coefficient $\frac{1}{4}$ in front of the second term can be eliminated by rescaling of time-variables: $T \rightarrow \frac{1}{2} T$, then the last term turns into $\frac{1}{16} T_{1}^{2} \delta_{n,-1}$.

We shall not actually discuss evaluation of the coefficient in front of $\mathcal{Z}$ (with no derivatives), which is denoted by [...] in (2.58) (see [24] and [30]). In fact almost all the terms in original complicated expression cancel, giving finally

$$
[\ldots]=\frac{1}{16 \lambda_{\gamma}^{2}}+\frac{T_{1}^{2}}{4 \lambda_{\gamma}}
$$

and this is represented by the terms with $\delta_{n, 0}$ and $\delta_{n,-1}$ in expressions (2.59) for the Virasoro generators $\mathcal{L}_{2 n}$.

The term with the double $T$-derivative in (2.58) is already of the necessary form. Of intermidiate complexity is evaluation of the coefficient in front of $\frac{\partial \mathcal{Z}}{\partial T_{2 a+1}}$ in (2.58), which we shall briefly describe now. First of all, rewrite this coefficient, reordering the items:

$$
\begin{array}{r}
\frac{1}{2}\left[\left(a+\frac{3}{2}\right) \lambda_{\gamma}^{-a-\frac{5}{2}}-\sum_{\delta \neq \gamma} \frac{1}{\lambda_{\gamma}-\lambda_{\delta}}\left(\lambda_{\gamma}^{-a-\frac{3}{2}}-\lambda_{\delta}^{-a-\frac{3}{2}}\right)\right]+ \\
+\left[\frac{1}{4} \lambda_{\gamma}^{-a-\frac{5}{2}}+\frac{1}{2} \sum_{\delta \neq \gamma} \frac{\lambda_{\gamma}^{-a-2}}{\sqrt{\lambda_{\delta}}+\sqrt{\lambda_{\gamma}}}\right]-\lambda_{\gamma}^{-a-1} .
\end{array}
$$

\footnotetext{
14 Therefore it could be reasonable to use a different notation: $\mathcal{L}_{n}$ instead of $\mathcal{L}_{2 n}$. We prefer $\mathcal{L}_{2 n}$, because it emphasises the property of the model to be 2-reduction of KP hierarchy (to KdV), see section 4 below.
} 
The first two terms together are equal to the sum over all $j$ (including $j=i$ ):

$$
\begin{aligned}
-\frac{1}{2} \sum_{\delta} \frac{1}{\lambda_{\gamma}-\lambda_{\delta}}\left(\lambda_{\gamma}^{-a-\frac{3}{2}}-\lambda_{\delta}^{-a-\frac{3}{2}}\right) & =\frac{1}{2} \sum_{\delta} \frac{\lambda_{\gamma}^{a+\frac{3}{2}}-\lambda_{\delta}^{a+\frac{3}{2}}}{\lambda_{\gamma}-\lambda_{\delta}} \cdot \frac{1}{\lambda_{\gamma}^{a+\frac{3}{2}} \lambda_{\delta}^{a+\frac{3}{2}}}= \\
& =\frac{1}{2 \lambda_{\gamma}^{a+2}} \sum_{\delta} \frac{\lambda_{\gamma}^{a+2}-\lambda_{\gamma}^{\frac{1}{2}} \lambda_{\delta}^{a+\frac{3}{2}}}{\lambda_{\gamma}-\lambda_{\delta}} \cdot \frac{1}{\lambda_{\delta}^{a+\frac{3}{2}}} .
\end{aligned}
$$

Similarly, the next two terms can be rewritten as

$$
\begin{array}{r}
\frac{1}{2} \sum_{\delta} \frac{\lambda_{\gamma}^{-a-2}}{\sqrt{\lambda_{\gamma}}+\sqrt{\lambda_{\delta}}}=\frac{1}{2 \lambda_{\gamma}^{a+2}} \sum_{\delta} \frac{\sqrt{\lambda_{\gamma}}-\sqrt{\lambda_{\delta}}}{\lambda_{\gamma}-\lambda_{\delta}}= \\
=\frac{1}{2 \lambda_{\gamma}^{a+2}} \sum_{\delta} \frac{\lambda_{\gamma}^{\frac{1}{2}} \lambda_{\delta}^{a+\frac{3}{2}}-\lambda_{\delta}^{a+2}}{\lambda_{\gamma}-\lambda_{\delta}} \cdot \frac{1}{\lambda_{\delta}^{a+\frac{3}{2}}} .
\end{array}
$$

The sum of these two expressions is equal to

$$
\frac{1}{2 \lambda_{\gamma}^{a+2}} \sum_{\delta} \frac{\lambda_{\gamma}^{a+2}-\lambda_{\delta}^{a+2}}{\lambda_{\gamma}-\lambda_{\delta}} \cdot \frac{1}{\lambda_{\delta}^{a+\frac{3}{2}}} .
$$

Note that powers $a+2$ are already integer and the remaining ratio can be represented as a sum of $a+2$ terms. Adding also the last term from the l.h.s. of (2.60), we finally obtain:

$$
\begin{array}{r}
-\frac{1}{\lambda_{\gamma}^{a+1}}+\frac{1}{2} \sum_{n=-1}^{a} \frac{1}{\lambda_{\gamma}^{n+2}} \sum_{\delta} \frac{1}{\lambda_{\delta}^{a-n+\frac{1}{2}}}= \\
=\frac{1}{2} \sum_{n=-1}^{a} \frac{1}{\lambda_{\gamma}^{n+2}}(2 a-2 n+1)(T+r)_{2 a-2 n+1}
\end{array}
$$

in accordance with (2.58) and (2.59).

\section{8 $\tilde{W}$-constraints for the asymmetric 2-matrix model}

We turn now to a very different application [38] of the GN equation (2.37).

Namely, we shall now consider $\mathcal{F}_{V, n}\{L\}$ as a building block in construction 
of conventional discrete two-matrix model

$$
\begin{array}{r}
Z_{N}\{t, \bar{t}\} \equiv c_{N}^{2} \int d H d \bar{H} e^{\sum_{k}\left(t_{k} \operatorname{Tr} H^{k}+\bar{t}_{k} \operatorname{Tr} \bar{H}^{k}\right)+\operatorname{Tr} H \bar{H}}= \\
=\int d L e^{\sum_{k} t_{k} \operatorname{Tr} L^{k}} \mathcal{F}_{\bar{U}, N}\{L\} .
\end{array}
$$

Now $L$ plays the role of $H$ and $\bar{U}(\bar{H})=\sum_{k} \bar{t}_{k} \bar{H}^{k}$.

We can now use GN equation to derive a relation for $Z_{N}\{t, \bar{t}\}$. Take (2.37),

$$
\left(\bar{U}\left(\frac{\partial}{\partial L_{t r}}\right)+L\right) \mathcal{F}_{\bar{U}, N}\{L\}=0
$$

multiply it by $e^{\operatorname{Tr} U(L)}=e^{\sum_{k} t_{k} \operatorname{Tr} L^{k}}$ and integrate over $L$. In order to express this relation in terms of $t$-derivatives of $z$ it is necessary to have some "scalar" rather than matrix equations, therefore we"ll actually need to take trace of (2.62). However, in order not to loose any information, we first multiply (2.62) by $L^{n}$ and then take the trace. In this way we obtain:

$$
\int d L e^{\sum_{k} t_{k} \operatorname{Tr} L^{k}} \operatorname{Tr} L^{n}\left(\bar{U}\left(\frac{\partial}{\partial L_{t r}}\right)+L\right) \mathcal{F}_{\bar{U}}\{L\}=0 .
$$

Integration by parts gives:

$$
\int d L \mathcal{F}_{\bar{U}}\{L\} \operatorname{Tr}\left(\bar{U}\left(-\frac{\partial}{\partial L_{t r}}\right)+L\right) L^{n} e^{\sum_{k} t_{k} \operatorname{Tr} L^{k}} .
$$

Now we need to introduce a new class of operators 38. Consider the action of $\operatorname{Tr} \frac{\partial^{m}}{\partial L_{t r}^{m}} L^{n}$ on $e^{\operatorname{Tr} U(L)}=e^{\sum_{k} t_{k} \operatorname{Tr} L^{k}}$. It gives some linear combination of terms like

$$
\operatorname{tr} L^{a_{1}} \ldots \operatorname{tr} L^{a_{l}} e^{\operatorname{tr} U(L)}=\frac{\partial^{l}}{\partial t_{a_{1}} \ldots \partial t_{a_{l}}} e^{-\operatorname{tr} U(L)}
$$

i.e. we obtain a combination of differential operators with $t$-derivatives, to be denoted $\tilde{W}(t)$ :

$$
\tilde{W}_{n-m}^{(m+1)}(t) e^{\operatorname{tr} U(L)} \equiv \operatorname{Tr} \frac{\partial^{m}}{\partial L_{t r}^{m}} L^{n} e^{\operatorname{tr} U(L)}, \quad m, n \geq 0 .
$$


For example,

$$
\begin{gathered}
\tilde{W}_{n}^{(1)}=\frac{\partial}{\partial t_{n}}, n \geq 0 \\
\tilde{W}_{n}^{(2)}=\sum_{k=0}^{\infty} k t_{k} \frac{\partial}{\partial t_{k+n}}+\sum_{k=0}^{n} \frac{\partial^{2}}{\partial t_{k} \partial t_{n-k}}, n \geq-1 ; \\
\tilde{W}_{n}^{(3)}=\sum_{k, l=1}^{\infty} k t_{k} l t_{l} \frac{\partial}{\partial t_{k+l+n}}+\sum_{k=1}^{\infty} k t_{k} \sum_{a+b=k+n} \frac{\partial^{2}}{\partial t_{a} \partial t_{b}}+ \\
+\sum_{k=1}^{\infty} k t_{k} \sum_{a+b=n+1} \frac{\partial^{2}}{\partial t_{a} \partial t_{b+k-1}}+\sum_{a+b+c=n} \frac{\partial^{3}}{\partial t_{a} \partial t_{b} \partial t_{c}}+\frac{(n+1)(n+2)}{2} \frac{\partial}{\partial t_{n}} ;
\end{gathered}
$$

Note, that while $\tilde{W}_{n}^{(1)}$ and $\tilde{W}_{n}^{(2)}$ are just the ordinary $(U(1)$-Kac Moody and Virasoro operators respectively, the higher $\tilde{W}^{(m)}$-operators do not coincide with the generators of the $\mathbf{W}$-algebras: already

$$
\begin{aligned}
\tilde{W}_{n}^{(3)} \neq W^{(3)}=\sum_{k, l=1}^{\infty} k t_{k} l t_{l} \frac{\partial}{\partial t_{k+l+n}}+ & 2 \sum_{k=1}^{\infty} k t_{k} \sum_{a+b=k+n} \frac{\partial^{2}}{\partial t_{a} \partial t_{b}} \\
& +\frac{4}{3} \sum_{a+b+c=n} \frac{\partial^{3}}{\partial t_{a} \partial t_{b} \partial t_{c}} .
\end{aligned}
$$

$\tilde{W}$-operators (in variance with ordinary $W$-operators) satisfy recurrent relation:

$$
\tilde{W}_{n}^{(m+1)}=\sum_{k=1}^{\infty} k t_{k} \tilde{W}_{n+k}^{(m)}+\sum_{k=0}^{m+n-1} \frac{\partial}{\partial t_{k}} \cdot \tilde{W}_{n-k}^{(m)}, \quad n \geq-m .
$$

Actually not too much is already known about the $\tilde{W}$ operators and the structure of $\tilde{\mathbf{W}}$-algebras (in particular it remains unclear whether the negative harmonics $\tilde{W}_{n}^{(m+1)}$ with $n<-m$ can be introduced in any reasonable way), see [38] for some preliminary results.

Equation (2.63) can now be represented in terms of the $\tilde{W}$-operators:

$$
\begin{array}{r}
\int d L \mathcal{F}_{\bar{U}}\{L\}\left(\sum_{k \geq 1} k \bar{t}_{k}\left(-\frac{\partial}{\partial L_{t r}}\right)^{k-1}+L\right) L^{n} e^{\operatorname{Tr} U(t)}= \\
=\left(\sum_{k \geq 1}(-)^{k-1} k \bar{t}_{k} \tilde{W}_{n+1-k}^{(k)}+\tilde{W}_{n+1}^{(1)}\right) Z_{N}\{t, \bar{t}\}=0 .
\end{array}
$$


This relation is highly asymmetric in $t$ and $\bar{t}$, and in fact it provides a suitable description of the WI only in the somewhat peculiar case when potential $\bar{U}(\bar{H})$ is a polinomial of finite degree. See refs. [57] and [38] for discussion of such asymmetric models.

\section{$2.9 \tilde{W}$-constraints for generic 2-matrix model}

When both potentials $U$ and $\bar{U}$ in (2.61) are generic formal seria, eqs.(2.67) represent only a one-parametric subset of the 2-parametric family of WI. Before we describe the whole set, let us emphasize that the two-matrix model (2.61) is the one, where the action is not of the most general form, consistent with some symmetry. Therefore it is not covariant under arbitrary change of variables $H, \bar{H} \longrightarrow f(H, \bar{H}), \bar{f}(H, \bar{H})$, and our usual method of derivation of Ward identities does not work. The reason why generic 2-matrix model with action containing all the possible combinations $\operatorname{Tr}\left(H^{a_{1}} \bar{H}^{b_{1}} H^{a_{2}} \bar{H}^{b_{2}} \ldots\right)$ is never considered seriously is essentially our poor understanding of the unitary-matrix interals for "non-eigenvalue" theories, to which class such generic model belongs. For reasons to be explained in the next section such problems do not arise for the models of the form (2.61) or (2.33), and this is why they attracted most attention so far. Hopefully the problems with the unitary-matrix integrals are temporal and this restricted class of multimatrix models will be unlarged, this should be especially easy to do in the part of the theory dealing with constraint algebras, but this subject is beyond the scope of the present notes.

In order to derive the complete set of WI for the model (2.61), we apply the following semi-artificial trick. Note that exponential $e^{\operatorname{Tr} H \bar{H}}$ satisfies:

$$
\left(\operatorname{Tr} H^{n} \frac{\partial^{m}}{\partial H_{t r}^{m}}-\operatorname{Tr} \bar{H}^{m} \frac{\partial^{n}}{\partial \bar{H}_{t r}^{n}}\right) e^{\operatorname{Tr} H \bar{H}}=0 .
$$

Let us integrate this identity over $H$ and $\bar{H}$ with the weight $e^{\operatorname{Tr} U(H)+\operatorname{Tr} \bar{U}(\bar{H})}$ and then integrate by parts. We obtain an identity:

$$
\begin{gathered}
\int d H d \bar{H} e^{\operatorname{Tr} H \bar{H}} \cdot \\
\cdot\left(\operatorname{Tr}\left(-\frac{\partial}{\partial H_{t r}}\right)^{m} H^{n}-\operatorname{Tr}\left(-\frac{\partial}{\partial \bar{H}_{t r}}\right)^{n} \bar{H}^{m}\right) e^{\operatorname{Tr} U(H)+\operatorname{Tr} \bar{U}(\bar{H})}=0,
\end{gathered}
$$


which can be represented in terms of $\tilde{W}$ operators [30]: 藏]

$$
\tilde{W}_{n-m}^{(m+1)}(t) Z\{t, \bar{t}\}=(-)^{m-n} \tilde{W}_{m-n}^{(n+1)}(\bar{t}) Z\{t, \bar{t}\}, \text { for all } m, n \geq 0 .
$$

This is the full(?) set of WI for the 2-matrix model. When one of potentials (say, $U(t)$ is polinomial of finite degree, the most of this symmetry is "spontaneously broken", the surviving part being described by eqs.(2.67).

Among other things eq.(2.70) reveals an amusing automorphsim of the $\tilde{\mathbf{W}}_{\infty}$ algebra:

$$
\tilde{W}_{n-m}^{(m+1)} \longleftrightarrow \tilde{W}_{m-n}^{(n+1)}, \quad m, n \geq 0,
$$

for example, Virasoro's Borel subalgebra is formed not only by operators $\tilde{W}_{n}^{(2)}$, but also by $\tilde{W}_{-n}^{(n+2)}, n \geq-1$ (while $U(1)$ Borel subalgebra - not only by $\tilde{W}_{n}^{(1)}=\frac{\partial}{\partial t_{n}}$, but also by $\left.\tilde{W}_{n}^{(n+1)}, n \geq 0\right)$.

One can attempt to apply the same procedure and derive $\tilde{W}$-identites for the conventional $(p-1)$-matrix models with $p-1>2$. In principle, this is possible, but unfortunately the arising equations neither have a nice form nor is there many enough of them. However, for illustrational purposes we shall scetch some relevant fromulas in the rest of this subsection.

Consider the multimatrix integral

$$
\begin{gathered}
Z=\int d H_{1} \ldots d H_{p-1} . \\
\cdot e^{\operatorname{Tr} U_{1}\left(H_{1}\right)+\ldots+\operatorname{Tr} U_{p-1}\left(H_{p-1}\right)} \ldots e^{\operatorname{Tr}\left(H_{1} H_{2}+H_{2} H_{3}+\ldots+H_{p-2} H_{p-1}\right)}
\end{gathered}
$$

Acting on $Z$, operator $\tilde{W}_{n-m}^{(m+1)}\left(t^{(1)}\right)$ produces an insertion of $\operatorname{Tr} H_{1}^{n}\left(\frac{\overleftarrow{\partial}}{\partial H_{1, t r}}\right)^{m}$ at the position of $\ldots$ in (2.72). Integration by parts gives:

$$
\operatorname{Tr} H_{1}^{n}\left(-\frac{\vec{\partial}}{\partial H_{1, t r}}\right)^{m} \longrightarrow(-)^{m} \operatorname{Tr} H_{1}^{n} H_{2}^{m}=(-)^{m} \operatorname{Tr} H_{2}^{m} H_{1}^{n}
$$

\footnotetext{
${ }^{15}$ Relations 2.68 ) and thus $\left(2.70\right.$ ) are in the obvious sense associated with $\operatorname{Tr} H^{n} \bar{H}^{m}$. Of course there are similar relations, in the same sense associated with any object like $\operatorname{Tr}\left(H^{a_{1}} \bar{H}^{b_{1}} H^{a_{2}} \bar{H}^{b_{2}} \ldots\right)$ and with products of such traces: it is enough to substitute all $\bar{H} \rightarrow \frac{\partial}{\partial H_{t r}}$ to obtain the l.h.s. of the equation and substitute all $H \rightarrow \frac{\partial}{\partial \bar{H}_{t r}}$ to obtain its r.h.s. (one should only remember that such substitution is possible, say in the l.h.s. if all the $\bar{H}$ are put to the right of all $H$, in order to restore the matrix-product form of the relation, one should carefully take into account all the commutators, arising when $\frac{\partial}{\partial H_{t r}}$ is carried back to original position of the corresponding $\bar{H}$ ). All such relations can appear to be just implications of eq. 2.70 .
} 
In the case of $p-1=2$, that we discussed above, this can be rewriten as $(-)^{m} \operatorname{Tr} H_{1}^{m}\left(\frac{\vec{\partial}}{\partial H_{2, t r}}\right)^{n}$ and integration by parts gives $(-)^{m} \operatorname{Tr} H_{2}^{m}\left(-\frac{\check{\partial}}{\partial H_{2, t r}}\right)^{n}$, what is equivalent to the action of $(-)^{m+n} \tilde{W}_{m-n}^{(n+1)}\left(t^{(2)}\right)$ on $Z$ : we reproduce equation (2.70).

However, for $p-1>2$ things are more complicated. Insertion of $\operatorname{Tr} H_{2}^{m} H_{1}^{n}$ is equivalent to that of $\operatorname{Tr} H_{2}^{m}\left(\frac{\vec{\partial}}{\partial H_{2, t r}}-H_{3}\right)^{n}$, which after integration by parts and acts on $e^{U_{2}\left(H_{2}\right)}$ and gives:

$$
\begin{gathered}
\operatorname{Tr} H_{2}^{m}\left(-\frac{\overleftarrow{\partial}}{\partial H_{2, t r}}-H_{3}\right)^{n} \sim \\
\sim \operatorname{Tr} H_{2}^{m}\left(\sum_{k} k t_{k}^{(2)} H_{2}^{k-1}-H_{3}\right)\left(-\frac{\overleftarrow{\partial}}{\partial H_{2, t r}}-H_{3}\right)^{n-1} \sim \ldots
\end{gathered}
$$

Derivatives remaining at the r.h.s. should be carried through the first bracket and than act on $e^{U_{2}\left(H_{2}\right)}$ etc. After all we get some linear combination of terms like $\operatorname{Tr} H_{2}^{b_{1}} H_{3}^{c_{1}} H_{2}^{b_{2}} H_{3}^{c_{2}} \ldots$ with $t^{(2)}$-dependent coefficients.

Now, if we are dealing with the $p-1=3$-matrix model, every $H_{2}$ standing to the right of all $H_{3}$ 's can be substituted by $\frac{\partial}{\partial H_{3, t r}}$, otherwise one should also include terms with commutators when this $\frac{\partial}{\partial H_{3, t r}}$ is carried back to the place where $\mathrm{H}_{2}$ was standing. In this way we obtain a combination of insertions of the form

$$
\begin{aligned}
& \operatorname{Tr}\left(\frac{\vec{\partial}}{\partial H_{3, t r}}\right)^{b_{1}} H_{3}^{c_{1}}\left(\frac{\vec{\partial}}{\partial H_{3, t r}}\right)^{b_{2}} H_{3}^{c_{2}} \ldots \sim \\
\sim & \operatorname{Tr}\left(-\frac{\overleftarrow{\partial}}{\partial H_{3, t r}}\right)^{b_{1}} H_{3}^{c_{1}}\left(-\frac{\overleftarrow{\partial}}{\partial H_{3, t r}}\right)^{b_{2}} H_{3}^{c_{2}} \ldots
\end{aligned}
$$

The resulting operator can be expressed through $\tilde{W}\left(t^{(3)}\right)$ and we obtain an identity, saying that some algebraic combination of $\tilde{W}\left(t^{(1)}\right)$ and $\tilde{W}\left(t^{(3)}\right)$ with $\left.t^{(2)}\right)$-dependent coefficients annihilates the partition function.

For $p-1>3$ insertion of $H_{2}$ is equivalent to that of $\frac{\partial}{\partial H_{3, t r}}-H_{4}$ rather than $\frac{\partial}{\partial H_{3, t r}}$, and the procedure should be repeated again and again. Finaly 
one arrives at constraints where the operators are algebraic combinations of $\tilde{W}\left(t^{(1)}\right)$ and $\tilde{W}\left(t^{(p-1)}\right)$ with the coefficients, which depend on $t^{(2)}, \ldots, t^{(p-2)}$ (moreover these are infinite seria in $\tilde{W}$ operators, unless all the intermediate potentials $U_{2}, \ldots, U_{p-2}$ are polinomials of finite degree.

This is of course not a too illuminating procedure and in fact it was never worked through to get concrete identities in any nice form. Instead it can serve to illustrate the problems, peculiar for the class of conventional multimatrix models (at least for $p-1>2$ ). It can also emphasize the beauty of conformal multimatrix models, which have clear advantages already at the level of Ward identites.

\subsection{0 $\tilde{W}$-operators in Kontsevich model}

After $\tilde{W}$-operators are introduced, we can also rewrite the GN equation (2.43) for Kontsevich models in terms of $\tilde{W}^{\prime}$ 's. Namely, we shall prove the following identity [38]:

$$
\left(\frac{\partial}{\partial \Lambda_{t r}}\right)^{m+1} \mathcal{Z}\left\{T_{k}\right\}=( \pm)^{m+1} \sum_{l \geq 0} \Lambda^{-l-1} \tilde{W}_{l-m}^{(m+1)}(T) \mathcal{Z}\left\{T_{k}\right\},
$$

valid for any function $\mathcal{Z}$ which depends on $T_{k}=\mp \frac{1}{k} \operatorname{tr} \Lambda^{-k}, \quad k \geq 1$ and $T_{0}= \pm \operatorname{tr} \log \Lambda$ with $n \times n$ matrix $\Lambda$. Application of the identity (2.75) is most straightforward in the Gaussian model (2.47), e.g. for transformation of eq. (2.50) into eq.(2.51) (we remind that $L=\Lambda$ in this case). In other cases calculations with the use of identity (2.75), accounting for the quasiclassical factor $\mathcal{C}_{V}\{L\}$ and the difference between $L=V^{\prime}(\Lambda)$ and $\Lambda$ become somewhat more involved, though still seem enough straightforward. Also for particular potentials $V(X)$ partition function $\mathcal{Z}_{V}\{T\}$ is actually independent of certain (combinations of) time-variables (for example, if $V(X)=\frac{X^{p+1}}{p+1}$ it is independent of all $T_{p k}, k \in Z_{+}$), and this is important for appearence of the constraints in the standard from like eqs.2.58), (2.59), i.e. for certain reduction of $\tilde{W}$-constraints to the ordinary $W$-constraints. This relation between $\tilde{W}$ - and $W$-operators deserves further investigation.

The proof of eq.(2.75) is provided by the following trick. Let us make a sort of Fourier transformation:

$$
\mathcal{Z}\{T\}=\int d H \mathcal{G}\{H\} e^{\sum_{k=0}^{\infty} T_{k} \operatorname{Tr} H^{k}},
$$


where integral is over $N \times N$ Hermitean matrix $H$. Then it is clear that once the identity (2.75) is established for $\mathcal{Z}\{T\}$ substituted by $e^{\operatorname{Tr} U(H)}, \quad U(H)=$ $\sum_{k=0}^{\infty} T_{k} \operatorname{Tr} H^{k}$, with any matrix $H$, it is valid for any function $\mathcal{Z}\{T\}$. The advantage of such substitution is that we can now make use of the definition (2.64) of the $\tilde{W}$ operators in order to rewrite (2.75) in a very explicit form:

$$
\begin{array}{r}
\left(\frac{\partial}{\partial \Lambda_{t r}}\right)^{m+1} e^{\operatorname{Tr} U(H)}=( \pm)^{m+1} \sum_{l \geq 0}^{\infty} \Lambda^{-l-1} \tilde{W}_{l-m}^{(m+1)}(T) e^{\operatorname{Tr} U(H)}= \\
=( \pm)^{m+1} \sum_{l \geq 0}^{\infty} \Lambda^{-l-1} \operatorname{Tr}\left(\frac{\partial}{\partial H_{t r}}\right)^{m} H^{l} e^{\operatorname{Tr} U(H)}= \\
=( \pm)^{m+1} \operatorname{Tr}\left(\frac{\partial}{\partial H_{t r}}\right)^{m} \frac{1}{\Lambda \otimes I-I \otimes H} e^{\operatorname{Tr} U(H)}
\end{array}
$$

Now expression for T's in terms of $\Lambda$ should be used. Then

$$
e^{\operatorname{Tr} U(H)}=\operatorname{Det}^{ \pm 1}(\Lambda \otimes I-I \otimes H)
$$

and substituting this into (2.77) we see that (2.75) is equivalent to

$$
\begin{array}{r}
\left(\left(\frac{\partial}{\partial \Lambda_{t r}}\right)^{m+1}-( \pm)^{m+1} I \cdot \operatorname{Tr}\left(\frac{\partial}{\partial H_{t r}}\right)^{m} \cdot \frac{1}{\Lambda \otimes I-I \otimes H}\right) \\
\cdot \operatorname{Det}^{ \pm 1}(\Lambda \otimes I-I \otimes H)=0
\end{array}
$$

Here "Tr" stands for the trace in the $H$-space only, while $D e t=\operatorname{Det} \otimes \operatorname{det}$ - for determinant in both $H$ and $\Lambda$ spaces. After one $\Lambda$-derivative is taken explicitly, we get:

$$
\begin{array}{r}
(I \otimes \operatorname{Tr})\left(\left(\frac{\partial}{\partial \Lambda_{t r}}\right)^{m} \otimes I-I \otimes\left( \pm \frac{\partial}{\partial H_{t r}}\right)^{m}\right) . \\
\cdot \frac{\operatorname{Det}^{ \pm 1}(\Lambda \otimes I-I \otimes H)}{\Lambda \otimes I-I \otimes H}=0 .
\end{array}
$$

This is already a matrix identity, valid for any $\Lambda$ and $H$ of the sizes $n \times n$ and $N \times N$ respectively. For example, if $m=0\left(\tilde{W}^{(1)}\right.$-case $)$, it is obviously

${ }^{16}$ Here it is for the first time that we encounter an important idea: matrix models the ordinary 1-matrix model (2.1) in this case - can be considered as defining integral transformations. This view on matrix models can to large extent define their role in the future developement of string theory. 
satisfied. If both $n=N=1$, it is also trivially true, though for different reasons for different choice of signs: for the upper signs, the ratio at the l.h.s. is just unity and all derivatives vansih; for the lower signs we have:

$$
\left(\frac{\partial}{\partial \lambda}\right)^{m}-\left(-\frac{\partial}{\partial h}\right)^{m}=\left(\sum_{\substack{a+b=m-1 \\ a, b \geq 0}}\left(\frac{\partial}{\partial \lambda}\right)^{a}\left(-\frac{\partial}{\partial h}\right)^{b}\right)\left(\frac{\partial}{\partial \lambda}+\frac{\partial}{\partial h}\right)
$$

and this obviously vanishes since $\left(\frac{\partial}{\partial \lambda}+\frac{\partial}{\partial h}\right) f(\lambda-h) \equiv 0$ for any $f(x)$.

If $m>0$ and $\Lambda, H$ are indeed matrices, direct evaluation becomes much more sophisticated. We present the first two nontrivial examples: $m=1$ and $m=2$. The following relartions will be usefull. Let $Q \equiv \frac{1}{\Lambda \otimes I-I \otimes H}$. Then

$$
\begin{aligned}
\operatorname{Det}^{ \pm 1} Q \frac{\partial}{\partial \Lambda_{t r}} D e t^{\mp 1} Q & = \pm[(I \otimes \operatorname{Tr}) Q] ; \\
\operatorname{Det}^{ \pm 1} Q \frac{\partial}{\partial H_{t r}} D e t^{\mp 1} Q & =\mp[(\operatorname{tr} \otimes I) Q] ; \\
\left(\frac{\partial}{\partial \Lambda_{t r}} \otimes I\right) Q & =-[(\operatorname{tr} \otimes I) Q] Q ; \\
\left(I \otimes \frac{\partial}{\partial H_{t r}}\right) Q & =[(I \otimes \operatorname{Tr}) Q] Q .
\end{aligned}
$$

This is already enough for the proof in the case of $m=1$. Indeed:

$$
\begin{aligned}
\operatorname{Det}^{ \pm 1} Q & \left(\frac{\partial}{\partial \Lambda_{t r}} \otimes I \mp I \otimes \frac{\partial}{\partial H_{t r}}\right) Q \operatorname{Det}^{\mp 1} Q= \\
= & \{-[(\operatorname{tr} \otimes I) Q] Q \pm[(I \otimes \operatorname{Tr}) Q] Q\} \mp \\
& \mp\{[(I \otimes \operatorname{Tr}) Q] Q \mp[(\operatorname{tr} \otimes I) Q] Q\}=0 .
\end{aligned}
$$

The first two terms at the r.h.s. come arise from $\Lambda$-, while the last two - from $H$-derivatives.

In the case of $m=2$ one should take derivatives once again. This is a little more tricky, and the same compact notation are not sufficient. In addition to (2.79) we now need:

$$
\left(\frac{\partial}{\partial \Lambda_{t r}} \otimes I\right)[(\operatorname{tr} \otimes I) Q] Q=-[(\operatorname{tr} \otimes I) Q]^{2} Q-\mathcal{B} .
$$


Here

$$
[(\operatorname{tr} \otimes I) Q]^{2}=[(\operatorname{tr} \otimes I)[(\operatorname{tr} \otimes I) Q] Q],
$$

while in order to write $\mathcal{B}$ explicitly we need to restore matrix indices (Greek for the $\Lambda$-sector and Latin - for the $H$ one). The $(\alpha i, \gamma k)$-component of (2.80) looks like:

$$
\left(\frac{\partial}{\partial \Lambda_{\beta \alpha}} \delta^{i m}\right) Q_{\delta \delta}^{m j} Q_{\beta \gamma}^{j k}=-Q_{\delta \delta}^{i j} Q_{\beta \beta}^{j l} Q_{\alpha \gamma}^{l k}-Q_{\delta \beta}^{i l} Q_{\alpha \delta}^{l j} Q_{\beta \gamma}^{j k}
$$

and appearence of the second term at the r.h.s. implies, that $\mathcal{B}_{\alpha \gamma}^{i k}=Q_{\delta \beta}^{i l} Q_{\alpha \delta}^{l j} Q_{\beta \gamma}^{j k}$. Further,

$$
\begin{aligned}
& \left(\frac{\partial}{\partial \Lambda_{t r}} \otimes I\right)[(I \otimes \operatorname{Tr}) Q] Q= \\
& -[(I \otimes \operatorname{Tr})[(\operatorname{tr} \otimes I) Q] Q] Q-[(I \otimes \operatorname{Tr})[(I \otimes \operatorname{Tr}) Q] Q] Q \\
& \left(I \otimes \frac{\partial}{\partial H_{t r}}\right)[(\operatorname{tr} \otimes I) Q] Q= \\
& +[(\operatorname{tr} \otimes I)[(I \otimes \operatorname{Tr}) Q] Q] Q+[(I \otimes \operatorname{Tr})[(\operatorname{tr} \otimes I) Q] Q] Q \\
& \left(I \otimes \frac{\partial}{\partial H_{t r}}\right)[(I \otimes \operatorname{Tr}) Q] Q=+[(I \otimes \operatorname{Tr})[(I \otimes \operatorname{Tr}) Q] Q] Q+\mathcal{B} .
\end{aligned}
$$

It is important that $\mathcal{B}$ that appears in the last relation in the form of $\mathcal{B}_{\alpha \gamma}^{i k}=$ $Q_{\alpha \delta}^{l j} Q_{\delta \beta}^{i l} Q_{\beta \gamma}^{j k}$ is exactly the same $\mathcal{B}$ as in eq.(2.80).

Now we can prove (2.78) for $m=2$ :

$$
\begin{aligned}
& \text { Det }^{ \pm 1} Q\left(\left(\frac{\partial}{\partial \Lambda_{t r}}\right)^{2} \otimes I-I \otimes\left(\frac{\partial}{\partial H_{t r}}\right)^{2}\right) Q D e t^{\mp 1} Q= \\
& =\{ \pm[(I \otimes \operatorname{Tr}) Q](-[(\operatorname{tr} \otimes I) Q] Q \pm[(I \otimes \operatorname{Tr}) Q] Q)- \\
& -(-[(\operatorname{tr} \otimes I)[(\operatorname{tr} \otimes I) Q] Q] Q-\mathcal{B}) \pm \\
& \pm(-[(I \otimes \operatorname{Tr})[(\operatorname{tr} \otimes I) Q] Q] Q-[(\operatorname{tr} \otimes I)[(I \otimes \operatorname{Tr}) Q] Q] Q)\}- \\
& -\{\mp[(\operatorname{tr} \otimes I) Q]([(I \otimes \operatorname{Tr}) Q] Q \mp[(\operatorname{tr} \otimes I) Q] Q)+ \\
& +([(I \otimes \operatorname{Tr})[(I \otimes \operatorname{Tr}) Q] Q] Q+\mathcal{B}) \mp \\
& \mp([(\operatorname{tr} \otimes I)[(I \otimes \operatorname{Tr}) Q] Q] Q+[(I \otimes \operatorname{Tr})[(\operatorname{tr} \otimes I) Q] Q] Q)\}
\end{aligned}
$$

where the terms $1,2,3,4,5,6$ in the first braces cancel the terms 1,3,2,4,6,5 in the second braces and identity (2.81) and its counterpart with $(\operatorname{tr} \otimes I) \rightarrow$ $(I \otimes \operatorname{Tr})$ is used.

Explicit proof of eq.(2.78) for generic $m$ is unknown. 


\section{Eigenvalue models}

\subsection{What are eigenvalue models}

Given the present state of knowledge we need to consider in most cases only the narrow class of the "eigenvalue" models. These models have the property of being associated with conventional integrable hierarchies (of (multicomponent) KP and Toda type), where integrable flows just commute (instead of forming less trivial closed algebras), and thus with the level-1 Kac-Moody algebras (by artificial tricks, familiar from the bosonization formalism in conformal field theory [58 these can be sometimes generalized to particular other levels like $k=2$ ). This means that the models are essentially associated with abelian Cartan subalgebras rather than with full matrix algebras. $\square$ In CFT-formulation (see below) this means that the eigenvalue models can be represented in terms of the free fields, which bosonize the Cartan subalgebra of the whole group in the WZNW model (the remaining $(\beta, \gamma)$-fields [16] being (almost) neglected - their remnants are observed in the form of "cocycle" factors in the Frenkel-Kac formulas [47], see [58]). In the matrix-integral representations the integrals for the eigenvalue models are in fact reduced to those over diagonal matrices (consisting of eigenvalues of original matrices, thus the name "eigenvalue models").

Most important, from the physical point of view eigenvalue models describe only topological (discrete) degrees of freedom, but not any propagating particles. 18 This can be understood if one notes that matrix models usually possess gauge symmetry, associated with the unitary rotation of matrices, $M_{\alpha} \longrightarrow U_{\alpha}^{\dagger} M_{\alpha} U_{\alpha}$, i.e. matrix models are usually gauge theories. In the case of eigenvalue models this symmetry is realized without "gauge fields" $V_{\alpha \beta}$, which would depend on pairs of indices $\alpha, \beta$ and transform like

\footnotetext{
${ }^{17}$ Groups, arising in the theory of matrix models and integrable hierarchies are not just those of matrices, appearing in the integral representations: the latter ones are in the best case related to the zero-modes of the former. Moreover, even this relation is not usually simple to reveal. This remark is important to avoid confusion in the next paragraphs.

18 Particles are always related to the "angular (unitary-) matrix" integrals (as everybody knows from example of the Wilson lattice QCD) which are highly less trivial to deal with, though these are also integrable in some broader sense of the word - within the (yet nonexisting) generalization of integrable hierarchies from the fields in the Cartan subalgebra to the entire WZNW model.
} 
$V_{\alpha \beta} \longrightarrow U_{\alpha}^{\dagger} V_{\alpha \beta} U_{\beta}$. In other words, eigenvalue models are gauge theories without gauge fields, i.e. are pure topological. Thus it is not a surprise that they usually live in the space time of dimension $d<2$, 9 since for $d>2$ there should be particles, associated with the gauge fields. At the "boundary" lies the model of " $d=2(c=1)$ string", which has one particle-like degree of freedom (dilaton, which becomes tachyon in the $d>2$ models). This very interesting model is much worse understood than the $d<2$ models, at least its properties are already somewhat different from other eigenvalue models (especially in the most interesting "compactified" case), and it will not be discussed in these notes. Later we"ll return to the subject of non-eigenvalue $(d>2)$ theories, though not too much is yet known about them, but now we are going to concentrate on the eigenvalue models.

\subsection{1-matrix model}

Hermitean matrix integrals are usually transformed to the eigenvalue form by separation of angular and eigenvalue variables. As usually, the simplest is the case of the 1-matrix model

$$
Z_{N}\{t\} \equiv c_{N} \int_{N \times N} d H e^{\sum_{k=0}^{\infty} t_{k} \operatorname{Tr} H^{k}}
$$

where this separations does not involve any information about unitary-matrix integrals. Take

$$
H=U^{\dagger} D U
$$

where $U$ is a unitary matrix and diagonal matrix $D=\operatorname{diag}\left(h_{1} \ldots h_{N}\right)$ has eigenvalues of $H$ as its entries. Then integration measure

$$
d H=\prod_{i, j=1}^{N} d H_{i j}=\frac{[d U]}{\left[d U_{\text {Cartan }}\right]} \prod_{i-1}^{N} d h_{i} \Delta^{2}(h),
$$

\footnotetext{
${ }^{19}$ Let us remind that in the Polyakov formulation which is the least counterintuitive for interpretation of what happens in the space-time (target space), string models usually involve Liouville field, identified as a time-variable in the target-space formalism. (Note that for this reason there is usually (at least one) time in the string theory, while space can be of any dimension (at least between 0 and 25), not obligatory integer.) Because of this extra Liouville field the space-time dimension $d$ usually differs by 1 from the central charge of the CFT model, which is coupled to $2 d$-gravity to from a string model: $d=c+1$ and $d<2$ is the same as $c<1$.
} 
where "Van-der-Monde determinant" $\Delta(h) \equiv \operatorname{det}_{(i j)} h_{i}^{j-1}=\prod_{i>j}^{N}\left(h_{i}-h_{j}\right)$ and $[d U]$ is Haar measure of integration over unitary matrices.

The way to derive eq.(3.3) is to consider the norm of infinitesimal variation

$$
\begin{aligned}
\|\delta H\|^{2} & \equiv \sum_{i, j=1}^{N}\left|\delta H_{i j}\right|^{2}=\sum_{i, j=1}^{N} \delta H_{i j} \delta H_{j i}=\operatorname{Tr}(\delta H)^{2}= \\
& =\operatorname{Tr}\left(-U^{\dagger} \delta U U^{\dagger} D U+U^{\dagger} D \delta U+U^{\dagger} \delta D U\right)^{2}= \\
& =\operatorname{Tr}(\delta D)^{2}+2 i \operatorname{Tr} \delta u[\delta D, D]+2 \operatorname{Tr}\left(-\delta u D \delta u D+(\delta u)^{2} D^{2}\right),
\end{aligned}
$$

where $\delta u \equiv \frac{1}{i} \delta U U^{\dagger}=\delta u^{\dagger}$ and $\delta D=\operatorname{diag}\left(\delta h_{1} \ldots \delta h_{N}\right)$. The second term at the r.h.s. vanishes because both $D$ and $\delta D$ are diagonal and commute. Therefore

$$
\|\delta H\|^{2}=\sum_{i=1}^{N}\left(\delta h_{i}\right)^{2}+\sum_{i, j=1}^{N}(\delta u)_{i j}(\delta u)_{j i}\left(h_{i}-h_{j}\right)^{2} .
$$

Now it remains to recall the basic relation between the infinitesimal norm and the measure: if $\|\delta l\|^{2}=G_{a b} \delta l^{a} \delta l^{b}$ then $[d l]=\sqrt{\operatorname{det}_{a b} G_{a b}} \prod_{a} d l^{a}$, to obtain eq.(3.3) with Haar measure $[d U]=\prod_{i j}^{N} d u_{i j}$ being associated with the infinitesimal norm

$$
\|\delta u\|^{2}=\operatorname{Tr}(\delta u)^{2}=\sum_{i, j=1}^{N} \delta u_{i j} \delta u_{j i}=\sum_{i, j=1}^{N}\left|\delta u_{i j}\right|^{2}
$$

and $\left[d U_{\text {Cartan }}\right] \equiv \prod_{i=1}^{N} d u_{i i}$.

Coming back to the 1-matrix model it remains to note that the "action" $\operatorname{Tr} U(H) \equiv \sum_{k=0}^{\infty} t_{k} \operatorname{Tr} H^{k}$ with $H$ substituted in the form (3.2) is independent of $U$ :

$$
\operatorname{Tr} U(H)=\sum_{i=1}^{N} U\left(h_{i}\right)
$$

Thus

$$
\begin{array}{r}
Z_{N}\{t\}=\frac{1}{N !} \prod_{i=1}^{N} \int d h_{i} e^{U\left(h_{i}\right)} \prod_{i>j}^{N}\left(h_{i}-h_{j}\right)^{2}= \\
=\frac{1}{N !} \prod_{i=1}^{N} \int d h_{i} e^{U\left(h_{i}\right)} \Delta^{2}(h),
\end{array}
$$


provided $c_{N}$ is chosen to be

$$
c_{N}^{-1}=N ! \frac{\operatorname{Vol}_{U(N)}}{\left(\operatorname{Vol}_{U(1)}\right)^{N}},
$$

where the volume of unitary group in Haar measure is equal to

$$
\mathrm{Vol}_{U(N)}=\frac{(2 \pi)^{N(N+1) / 2}}{\prod_{k=1}^{N} k !} .
$$

A simple way to derive eq.(3.6) will be described at the end of this section, as an example of application of orthogonal polinomials technique.

\subsection{Itzykson-Zuber and Kontsevich integrals}

Let us proceed now to Kontsevich integral,

$$
\mathcal{F}_{V, n}\{L\}=\int_{n \times n} d X e^{-\operatorname{tr} V(X)+\operatorname{tr} L X} .
$$

We shall see shortly that it in fact depends only on the eigenvalues of the matrix $L$ (this fact was already used in the previous section), however, this time somewhat more sophisticated unitary matrix integrals will be involved.

Substitute $X=U_{X}^{\dagger} D_{X} U_{X} ; \quad L=U_{L}^{\dagger} D_{L} U_{L}$ in (3.7) and denote $U \equiv U_{X} U_{L}^{\dagger}$. Then

$$
\begin{aligned}
& \mathcal{F}_{V, n}\{L\}= \\
& =\prod_{i=1}^{n} \int d x_{i} e^{-V\left(x_{i}\right)} \Delta^{2}(x) \int_{n \times n} \frac{[d U]}{\left[d U_{\text {Cartan }}\right]} \exp \left(\sum_{\gamma, \delta=1}^{n} x_{\gamma} l_{\delta}\left|U_{\gamma \delta}\right|^{2}\right) .
\end{aligned}
$$

In order to proceed further we need to evaluate the integral over unitary matrices, which appeared at the r.h.s.

This integral can actually be represented in two different ways:

$$
\begin{array}{r}
I_{n}\{X, L\} \equiv \int_{n \times n} \frac{[d U]}{\left[d U_{\text {Cartan }}\right]} e^{\operatorname{tr} X U L U^{\dagger}}= \\
=\int_{n \times n} \frac{[d U]}{\left[d U_{\text {Cartan }}\right]} e^{\sum_{\gamma, \delta=1}^{n} x_{\gamma} l_{\delta}\left|U_{\gamma \delta}\right|^{2}}
\end{array}
$$


(the U's in the two integrals are related by the transformation $U \longrightarrow U_{X} U U_{L}^{\dagger}$ and Haar measure is both left and right invariant). Formula (3.9) implies that $I_{n}\{X, L\}$ satisfies a set of simple equations [59]:

$$
\begin{aligned}
& \left(\operatorname{tr}\left(\frac{\partial}{\partial X_{t r}}\right)^{k}-\operatorname{tr} L^{k}\right) I_{n}\{X, L\}=0, \quad k \geq 0, \\
& \left(\operatorname{tr}\left(\frac{\partial}{\partial L_{t r}}\right)^{k}-\operatorname{tr} X^{k}\right) I_{n}\{X, L\}=0, \quad k \geq 0,
\end{aligned}
$$

which by themselves are not very restrictive. However, another formula, (3.10), implies that $I_{n}\{X, L\}$ in fact depends only on the eigenvalues of $X$ and $L$, and for such $I_{n}\{X, L\}=\hat{I}\left\{x_{\gamma}, l_{\delta}\right\}$ eqs.(3.11) become very restrictive 20 and allow to determine $\hat{I}\left\{x_{\gamma}, l_{\delta}\right\}$ unambigously (at least if $\hat{I}\left\{x_{\gamma}, l_{\delta}\right\}$ is expandable in a formal power seria in $x_{\gamma}$ and $l_{\delta}$ ). The final solution is

$$
I_{n}\{X, L\}=\frac{(2 \pi)^{\frac{n(n-1)}{2}}}{n !} \frac{\operatorname{det}_{\gamma \delta} e^{x_{\gamma} l_{\delta}}}{\Delta(x) \Delta(l)} .
$$

Normalization constant can be defined by taking $L=0$, when

$$
I_{n}\{X, L=0\}=\frac{\mathrm{Vol}_{U(n)}}{\left(\mathrm{Vol}_{U(1)}\right)^{n}}=\frac{(2 \pi)^{\frac{n(n-1)}{2}}}{\prod_{k=1}^{n} k !},
$$

and using the fact that

$$
\left.\frac{\operatorname{det}_{\gamma \delta} f_{\gamma}\left(l_{\delta}\right)}{\Delta(l)}\right|_{\left\{l_{\delta}=0\right\}}=\left(\prod_{k=0}^{n-1} \frac{1}{k !}\right) \operatorname{det}_{\gamma \delta} \partial^{\delta-1} f_{\gamma}(0) .
$$

Eq.(3.12) is usually refered to as the Itzykson-Zuber formula [60]. In mathematical literature it was earlier derived by Kharish-Chandra [61, and

${ }^{20}$ When acting on $\hat{I}$, which depends only on eigenvalues, matrix derivatives turn into:

$$
\begin{aligned}
& \operatorname{tr} \frac{\partial}{\partial X_{t r}} \hat{I}=\sum_{\gamma} \frac{\partial}{\partial x_{\gamma}} \hat{I} \\
& \operatorname{tr} \frac{\partial^{2}}{\partial X_{t r}^{2}} \hat{I}=\sum_{\gamma} \frac{\partial^{2}}{\partial x_{\gamma}^{2}} \hat{I}+\sum_{\gamma \neq \delta} \frac{1}{x_{\gamma}-x_{\delta}}\left(\frac{\partial}{\partial x_{\gamma}}-\frac{\partial}{\partial x_{\delta}}\right) \hat{I}
\end{aligned}
$$

etc. 
in fact the integral (3.9 is the basic example of the coadjoint orbit integrals [62]- [5], which can be exactly evaluated with the help of the DuistermaatHeckmann theorem [64], [65], [43], [44]. This calculation is the simplest example of the very important technique of exact evaluation of non - Gaussian unitary-matrix integrals, which is now doing its first steps (see 66- 68 ) and will be discussed at the end of these notes.

Now we turn back to the eigenvalue formulation of the GKM. Substitution of (3.12) into (3.8) gives:

$$
\begin{array}{r}
\mathcal{F}_{V, n}\{L\}=\frac{(2 \pi)^{\frac{n(n-1)}{2}}}{\Delta(l)} \prod_{\delta=1}^{n} \int d x_{\delta} e^{-V\left(x_{\delta}\right)} \Delta(x) \frac{1}{n !} \operatorname{det}_{\gamma \delta} e^{x_{\gamma} l_{\delta}}= \\
=\frac{(2 \pi)^{\frac{n(n-1)}{2}}}{\Delta(l)} \prod_{\delta=1}^{n} \int d x_{\delta} e^{-V\left(x_{\delta}\right)+x_{\delta} l_{\delta}} \Delta(x),
\end{array}
$$

where we used antisymmetry of $\Delta(x)$ under permutations of $x_{\gamma}$ 's in order to change $\frac{1}{n !} \operatorname{det}_{\gamma \delta} e^{x_{\gamma} l_{\delta}}$ for $e^{\sum_{\delta} x_{\delta} l_{\delta}}$ under the sign of the $x_{\delta}$ integration.

We can now use the fact that $\Delta(x)=\operatorname{det}_{\gamma \delta} x_{\delta}^{\gamma-1}$ in order to rewrite the r.h.s. of $(3.13)$ :

$$
\mathcal{F}_{V, n}\{L\}=(2 \pi)^{\frac{n(n-1)}{2}} \frac{\operatorname{det}_{\gamma \delta} \hat{\varphi}_{\gamma}\left(l_{\delta}\right)}{\Delta(l)}
$$

where

$$
\hat{\varphi}_{\gamma}(l) \equiv \int d x x^{\gamma-1} e^{-V(x)+l x}, \quad \gamma \geq 1
$$

These functions $\hat{\varphi}(l)$ satisfy a simple recurrent relation:

$$
\hat{\varphi}_{\gamma}=\frac{\partial \hat{\varphi}_{\gamma-1}}{\partial l}=\left(\frac{\partial}{\partial l}\right)^{\gamma-1} \hat{\Phi}
$$

with

$$
\hat{\Phi}(l) \equiv \hat{\varphi}_{1}(l)=\int d x e^{-V(x)+l x} .
$$

Note also that if the "zero-time" $N$ is introduced (see subsection 2.6 above and [36]), then

$$
\mathcal{F}_{V, n}\{N \mid L\} \equiv \mathcal{F}_{V(X)-N \log X, n}\{L\}=(2 \pi)^{\frac{n(n-1)}{2}} \frac{\operatorname{det}_{\gamma \delta} \hat{\varphi}_{\gamma+N}\left(l_{\delta}\right)}{\Delta(l)}
$$


with just the same $\hat{\varphi}_{\gamma}(l)$ and $\gamma, \delta=1 \ldots n$. If we divide by the quasiclassical factor $\mathcal{C}_{V}\{\Lambda\}(\operatorname{det} \Lambda)^{N}, L=V^{\prime}(\Lambda)$, in order to transform Kontsevich integral into Kontsevich model (see section 2.5), we get:

$$
\mathcal{Z}_{V}\{N, T\}=\frac{1}{(\operatorname{det} \Lambda)^{N}} \cdot \frac{\operatorname{det}_{\gamma \delta} \varphi_{\gamma+N}\left(\lambda_{\delta}\right)}{\Delta(\lambda)} .
$$

The role of $\mathcal{C}_{V}\{\Lambda\}$ is to convert $\hat{\varphi}(l)$ into the properly normalized expansions in the negative iteger powers of $\lambda$ :

$$
\varphi_{\gamma}(\lambda)=\frac{e^{-\lambda V^{\prime}(\lambda)+V(\lambda)} \sqrt{V^{\prime \prime}(\lambda)}}{\sqrt{2 \pi}} \hat{\varphi}_{\gamma}\left(V^{\prime}(\lambda)\right)=\lambda^{\gamma-1}\left(1+\mathcal{O}\left(\lambda^{-1}\right)\right),
$$

and to change $\Delta(l)=\Delta\left(V^{\prime}(\lambda)\right)$ in the denominator of (3.18) for $\Delta(\lambda)$ in (3.19). Instead of the simple recurrent relations (3.16) for $\hat{\varphi}$ the normalized functions $\varphi$ satisfy:

$$
\varphi_{\gamma}(\lambda)=\mathcal{A} \varphi_{\gamma-1}(\lambda)=\mathcal{A}^{\gamma-1} \Phi(\lambda)
$$

where $\Phi(\lambda)=\varphi_{1}(\lambda)$ and operator

$$
\mathcal{A}=\frac{1}{V^{\prime \prime}(\lambda)} \cdot \frac{\partial}{\partial \lambda}-\frac{1}{2} \frac{V^{\prime \prime \prime}(\lambda)}{\left(V^{\prime \prime}(\lambda)\right)^{2}}+\lambda
$$

now depends on the potential $V(x)$.

\subsection{Conventional Multimatrix models}

The multimatrix integrals of the form

$$
\equiv c_{N}^{p-1} \int_{N \times N} d H^{(1)} \ldots d H^{(p-1)} \prod_{\alpha=1}^{p-1} e^{\sum_{k=0}^{\infty} t_{k}^{(\alpha)} \operatorname{Tr} H_{(\alpha)}^{k}} \prod_{\alpha=1}^{p-2} e^{Z_{N}\left\{t^{(\alpha)}\right\} \equiv}
$$

can be rewritten in the eigenvalue form using the same Itzykson-Zuber formula (3.12). Indeed, substituting $H^{(\alpha)}=U^{(\alpha)}{ }^{\dagger} D^{(\alpha)} U^{\alpha)}$ and then defyining 
$U^{(\alpha)} U^{(\alpha+1)^{\dagger}} \equiv \tilde{U}^{(\alpha)}$, we obtain:

$$
\begin{gathered}
Z_{N}\left\{t^{(\alpha)}\right\}= \\
=\frac{1}{N !} \prod_{\alpha=1}^{p-1} \prod_{i=1}^{N} \int d h_{i}^{(\alpha)} e^{-V\left(h_{i}^{(\alpha)}\right)} \Delta^{2}\left(h^{(\alpha)}\right) \prod_{\alpha=1}^{p-2} I_{N}\left\{H^{(\alpha)}, H^{(\alpha+1)}\right\}= \\
=\frac{1}{N !} \prod_{\alpha=1}^{p-1} \prod_{i=1}^{N} \int d h_{i}^{(\alpha)} e^{-V\left(h_{i}^{(\alpha)}\right)} \prod_{\alpha=1}^{p-2} e^{h_{i}^{(\alpha)} h_{i}^{(\alpha+1)}} \Delta\left(h^{(1)}\right) \Delta\left(h^{(2)}\right),
\end{gathered}
$$

where the same trick is done with the substitution of $\frac{1}{N !} \operatorname{det}_{i j} e^{h_{i}^{(\alpha)} h_{j}^{(\alpha+1)}}$ for $e^{\sum_{i=1}^{N} h_{i}^{(\alpha)} h_{i}^{(\alpha+1)}}$ under the sign of the $h_{i}^{(\alpha)}$-integration (step by step: first for $\alpha=1$, then for $\alpha=2$ and so on). Note that all the Van-der-Monde determinants disappeared from the final formula at the r.h.s. of eq.(3.24), except for those at the ends of the matrix chain (at $\alpha=1$ and $\alpha=p-1$ ).

If the chain was closed rather than open, i.e. there was an additional factor of $e^{\operatorname{Tr} H^{(p-1)} H^{(1)}}$ under the integral in (3.23), then the trick with separation of all angular-variable (unitary-matrix) integrations would not work so simply: in addition to the Itzykson-Zuber integral the much more involved quantities would be required, like

$$
\begin{gathered}
I_{n}\left\{X_{1}, X_{2} ; L\right\} \equiv \\
\equiv c_{n} \int_{n \times n} \frac{\left[d U_{1}\right]}{\left[d U_{1, \text { Cartan }}\right]} \frac{\left[d U_{2}\right]}{\left[d U_{2, \text { Cartan }}\right]} \\
\cdot \exp \left(\operatorname{tr} X_{1} U_{1} L U_{1}^{\dagger}+\operatorname{tr} X_{2} U_{2} L U_{2}^{\dagger}+\operatorname{tr} X_{1}\left(U_{1} U_{2}^{\dagger}\right) X_{2}\left(U_{2} U_{1}^{\dagger}\right)\right)
\end{gathered}
$$

This (so far unresolved) closed chain model (lattice Potts model) is an example of non-eigenvalue models, in the $p=\infty$ case it turns into "compactified" $c=1$ model. This theory is more complicated then the so far simplest class of non-eigenvalue models of "induces Yang-Mills theory", known as KazakovMigdal models.

\subsection{Determinant formulas for eigenvalue models}

We are now prepared to make the crucial step towards understanding of mathematical structure behind eigenvalue models, which distinguishes their partition functions in the entire variety of arbitrary $N$-fold integrals. This 
structure expresses itself in the form of determinantal formulas, which we are now going to discuss. In the next section 4 these formulas will be identified as examples of $\tau$-functions of KP and Toda hierarchies.

Looking at the relevant integrals (3.4), (3.24) one can notice that integrals over different eigenvalues with non-trivial measures which depend on the shape of potentials $U$ or $V$, are almost separated, the only "interaction" between different eigenvalues being defined by universal (potentialindependent) quantities, made from the Van-der-Monde determinants. This feature is intimately related both to its origin (decoupling of angular variables in original matrix integral) and to its most important implication (integrability). The main property of the Van-der-Monde determinant is that it is at the same time a Pfaffian (and it is in this quality that it arises from matrix integrals) and a determinant (and this is the feature that implies integrability):

$$
\prod_{i>j}\left(h_{i}-h_{j}\right)=\Delta(h)=\operatorname{det}_{i j} h_{i}^{j-1}
$$

We already used this property above, when going from eq.(3.13) to eq.(3.14), which as we shall see later is the crucial step in the proof of integrability of Kontsevich model. In that case determinantal formula (3.14) for partition function was trivial to derive, because the integrand was linear in Van-derMonde determinants. Now we turn to slightly more complicated situations, involving products of Van-der-Monde determinants.

Consider an eigenvalue model of the form:

$$
Z_{N}=\frac{1}{N !} \prod_{k=1}^{N} \int d \mu_{h_{k}, \bar{h}_{k}} \Delta(h) \Delta(\bar{h})
$$

to be refered to as "scalar-product" model. All conventional multimatrix models (3.23) belong to this class. In the case of the 1-matrix model (3.4)

$$
d \mu_{h, \bar{h}}=d h d \bar{h} e^{U(h)} \delta(h-\bar{h}),
$$

while for conventional multimatrix models (3.24)

$$
d \mu_{h^{(1)}, h^{(p-1)}}=d h^{(1)} d h^{(p-1)} \prod_{\alpha=2}^{p-2} \int d h^{(\alpha)} \prod_{\alpha=1}^{p-1} e^{U_{\alpha}\left(h^{(\alpha)}\right)} \prod_{\alpha=1}^{p-2} e^{h^{(\alpha)} h^{(\alpha+1)}}
$$


If $d \mu_{h, \bar{h}}=\delta(h-\bar{h}) d \bar{h} d \mu_{h}$ we call this measure local. The main feature of local measure is that operator of multiplication by $H$ (or any function of $h$ ) is Hermitean. Thus measure is local in the 1-matrix model, but is non-local for all $p-1>1$. In the latter case the measure is defined to depend only on $h=h^{(1)}$ and $\bar{h}=h^{(p-1)}$, all other $h^{(\alpha)}, \alpha=2 \ldots p-2$ being integrated out, what makes the "interaction" between $h$ and $\bar{h}$ more complicated than just $\delta(h-\bar{h})$ in the one-matrix $(p=2)$ and $e^{h \bar{h}}$ in the two-matrix $(p=3)$ cases. In no sense the set of particular formulas (3.29) for $p>3$ is distinguished among other scalar-product models, and from now on we shall not consider conventional multimatrix models with $p-1>2$ as a separate class of theories.

Eqs.(3.27) and (3.26) imply together that

$$
\begin{aligned}
& Z_{N}=\frac{1}{N !} \prod_{k=1}^{N} \int d \mu_{h_{k}, \bar{h}_{k}} \operatorname{Det}_{i k} h_{k}^{i-1} \operatorname{Det}_{j k} \bar{h}_{k}^{j-1}= \\
& =\operatorname{Det}_{i j} \int d \mu_{h, \bar{h}} h^{i-1} \bar{h}^{j-1}=\operatorname{Det}_{i j}\left\langle h^{i-1} \mid \bar{h}^{j-1}\right\rangle,
\end{aligned}
$$

where an obvious notation is introduced for the scalar product

$$
\langle f(h) \mid g(\bar{h})\rangle \equiv \int d \mu_{h, \bar{h}} f(h) g(\bar{h}) .
$$

We can now be a little more specific and introduce time-variables $t_{k}$ and $\bar{t}_{k}$ so, that

$$
\begin{array}{r}
d \mu_{h, \bar{h}}=e^{U(h)+\bar{U}(\bar{h})} d \hat{\mu}_{h, \bar{h}}, \\
U(h)=\sum_{k=-\infty}^{\infty} t_{k} h^{k}, \quad \bar{U}(\bar{h})=\sum_{k=-\infty}^{\infty} \bar{t}_{k} \bar{h}^{k},
\end{array}
$$

and $\hat{d \mu} \mu_{h, \bar{h}}$ is already independent of $h$ and $\bar{h}$. If we now denote $\mathcal{H}^{f}(t, \bar{t}) \equiv$ $\langle 1 \mid 1\rangle$, then

$$
\begin{array}{r}
\mathcal{H}_{i j}^{f} \equiv\left\langle h^{i} \mid \bar{h}^{j}\right\rangle=\frac{\partial^{2}}{\partial t_{i} \partial \bar{t}_{j}} \mathcal{H}^{f}(t, \bar{t})= \\
\quad \text { if } \stackrel{i, j}{\underline{\underline{n}} \geq 0}\left(\frac{\partial}{\partial t_{1}}\right)^{i}\left(\frac{\partial}{\partial \bar{t}_{1}}\right)^{j} \mathcal{H}^{f}(t, \bar{t}),
\end{array}
$$

and

$$
Z_{N}=\operatorname{Det}_{N} \mathcal{H}_{i j}^{f}
$$


where $\operatorname{Det}_{N}$ stands for determinant of the $N \times N$ matrix $\mathcal{H}_{i-1, j-1}$ (which is defined itself for any integers $i, j$ ) with $i, j=0, \ldots, N-1$. Characteristic property of $\mathcal{H}_{i j}^{f}$ is its peculiar time-dependence:

$$
\frac{\mathcal{H}_{i j}^{f}}{\partial t_{k}}=\mathcal{H}_{i+k, j}^{f} ; \quad \frac{\mathcal{H}_{i j}^{f}}{\partial \bar{t}_{k}}=\mathcal{H}_{i, j+k}^{f} .
$$

Eq.(3.33) provides the determinantal formula for all scalar-product models. The case of the local measure - for 1-matrix model - is a little special. In this case $U(h)$ contains the whole information about the measure: $d \mu_{h, \bar{h}}=\delta(h-\bar{h}) d \mu_{h}, d \mu_{h}=e^{U(h)} d h$, and there is no $\bar{U}(\bar{h})$ (or $\bar{t}$ simply coincide with $t$ ). Then (3.33) is still valid, but

$$
\begin{array}{r}
\mathcal{H}_{i j}^{f}=\left.\left\langle h^{i} \mid \bar{h}^{j}\right\rangle\right|_{d \mu_{h, \bar{h}}}=\left.\left\langle h^{i+j}\right\rangle\right|_{d \mu_{h}}=\frac{\partial}{\partial t_{i+j}} \mathcal{H}^{f}(t)= \\
\stackrel{\text { if } \stackrel{i, j}{=} \geq 0}{\left(\frac{\partial}{\partial t_{1}}\right)^{i+j} \mathcal{H}^{f}(t) .}
\end{array}
$$

The same formula (3.35) can be also derived as a limit of eq.(3.14) for Kontsevich integral. Indeed,

$$
\begin{array}{r}
Z_{N}\{t\}=c_{N} \int_{N \times N} d H e^{\operatorname{Tr} U(H)}=\lim _{L \rightarrow 0} \mathcal{F}_{U, N}\{L\}= \\
=\lim _{\left\{l_{j}\right\} \rightarrow 0} \frac{\operatorname{Det}_{i j} \hat{\varphi}_{i}^{\{U\}}\left(l_{j}\right)}{\Delta(l)}=\operatorname{Det}_{i j} \frac{\partial^{j-1} \hat{\varphi}_{i}^{\{U\}}\left(l_{j}\right)}{\partial l^{j-1}}(0)=\operatorname{Det}_{i j} \mathcal{H}_{i-1, j-1}^{f},
\end{array}
$$

where this time

$$
\left.\mathcal{H}_{i-1, j-1}^{f} \stackrel{i, j>0}{=} \frac{\partial^{j-1} \hat{\varphi}_{i}^{\{U\}}\left(l_{j}\right)}{\partial l^{j-1}}(l=0) \stackrel{(3.14)}{=}\left(\frac{\partial}{\partial l}\right)^{i+j-2} \hat{\Phi}^{\{U\}}\right|_{l=0} .
$$

Now we note, that the action of $\frac{\partial}{\partial l}$ on $\hat{\Phi}^{\{U\}}(l)=\int d x e^{U(x)+l x}$ is equivalent to that of $\left(\frac{\partial}{\partial t_{1}}\right)$, since this is no longer a matrix integral, and thus

$$
\mathcal{H}_{i j}^{f}=\left(\frac{\partial}{\partial t_{1}}\right)^{i+j} \hat{\Phi}^{\{U\}}(0)
$$

i.e. $\mathcal{H}^{f}(t)=\hat{\Phi}^{\{U\}}(0)$. 
Conformal multimatrix models were introduced in section 2.3 above just as eigenvalue models. For the $A_{p-1}$ series partition functions are defined to be

$$
\begin{gathered}
Z_{N_{1} \ldots N_{p-1}}^{A_{p-1}}\left\{t^{(1)} \ldots t^{(p-1)}\right\}= \\
=\prod_{\alpha=1}^{p-1} c_{N_{\alpha}} \int_{N_{\alpha} \times N_{\alpha}} d H^{(\alpha)} e^{\operatorname{Tr} U_{\alpha}\left(H^{(\alpha)}\right)} . \\
\cdot \prod_{\alpha=1}^{p-2} \operatorname{Det}\left(H^{(\alpha)} \otimes I-I \otimes H^{(\alpha+1)}\right)= \\
=\prod_{\alpha=1}^{p-1} \frac{1}{N_{\alpha} !} \prod_{i=1}^{N_{\alpha}} \int d h_{i}^{(\alpha)} e^{U_{\alpha}\left(h_{i}^{(\alpha)}\right)} \Delta^{2}\left(h^{(\alpha)}\right) \prod_{\alpha=1}^{p-2} \prod_{i, k}\left(h_{i}^{(\alpha)}-h_{k}^{(\alpha+1)}\right) .
\end{gathered}
$$

This expression does not have a form of eq.(3.27), thus conformal matrix models for $p-1>1$ are not of the "scalar-product" type. We shall sometimes call them $(p-1)$-component models, because they are related to the multicomponent integrable hierarchies. The simplest way to proceed with their investigation is to use the same trick with Kontsevich integral, which was just applied in the 1-matrix case.

Let us start from a very general $(p-1)$-component model:

$$
Z=\prod_{\alpha=1}^{p-1} \int_{N_{\alpha} \times N_{\alpha}} d H^{(\alpha)} e^{\operatorname{Tr} U_{\alpha}\left(H^{(\alpha)}\right)} K\left(H^{(1)} \ldots H^{(p-1)}\right) .
$$

It can be also represented in terms of Kontsevich integrals:

$$
Z=\left.K\left(\frac{\partial}{\partial L_{t r}^{(1)}}, \ldots, \frac{\partial}{\partial L_{t r}^{(p-1)}}\right) \prod_{\alpha=1}^{p-1} \mathcal{F}_{U_{\alpha}, N_{\alpha}}\left\{L^{(\alpha)}\right\}\right|_{L^{(\alpha)}=0}
$$

This representation is not very usefull, since the limit $L \rightarrow 0$ is not easy to take, unless $K$ is a polinomial in the eigenvalues of all its arguments. However, this is exactly the case for our confromal models (3.39). Indeed,

$$
K^{A_{p-1}}=\prod_{\alpha=1}^{p-2} \operatorname{Det}\left(\frac{\partial}{\partial L_{t r}^{(\alpha)}} \otimes I-I \otimes \frac{\partial}{\partial L_{t r}^{(\alpha+1)}}\right) .
$$


Still this is not very convenient, because representation (3.14) for $\mathcal{F}$ contain $\Delta(L)$ in denominator, which are not very pleasant to differentiate. Simplification can be achieved if instead we rewrite the original expression at the r.h.s. of (3.39) as follows:

$$
\begin{aligned}
& Z_{N_{1} \ldots N_{p-1}}^{A_{p-1}}\left\{t^{(1)} \ldots t^{(p-1)}\right\}= \\
& =\Delta\left(\frac{\partial}{\partial l^{(1)}}\right) \prod_{\alpha=1}^{p-2} \Delta\left(\frac{\partial}{\partial l^{(\alpha)}}, \frac{\partial}{\partial l^{(\alpha+1)}}\right) \Delta\left(\frac{\partial}{\partial l^{(p-1)}}\right) \times \\
& \times\left.\prod_{\alpha=1}^{p-1}\left(\frac{1}{N_{\alpha} !} \prod_{i=1}^{N_{\alpha}} \int d h_{i}^{(\alpha)} e^{U_{\alpha}\left(h_{i}^{(\alpha)}\right)+l_{i}^{(\alpha)} h_{i}^{(\alpha)}}\right)\right|_{l^{(\alpha)}=0}
\end{aligned}
$$

where $\Delta\left(h, h^{\prime}\right) \equiv \prod_{i>j}^{N}\left(h_{i}-h_{j}\right) \prod_{k>l}^{N^{\prime}}\left(h_{k}^{\prime}-h_{l}^{\prime}\right) \prod_{i=1}^{N} \prod_{k=1}^{N^{\prime}}\left(h_{k}^{\prime}-h_{i}\right)$. This formula already takes the specific form of $K$ into account. The product of integrals in brackets at the r.h.s. of $(3.43)$ is equal (for every fixed $\alpha$ ) to

$$
\frac{1}{N_{\alpha} !} \prod_{j=1}^{N_{\alpha}} \hat{\Phi}^{\left\{U_{\alpha}\right\}}\left(l_{j}^{(\alpha)}\right)
$$

(compare with eq.(3.38)).

In order to simplify the notation we shall further denote $\hat{\Phi}^{\left\{U_{\alpha}\right\}}(l) \equiv$ $\int d x e^{U_{\alpha}(x)+l x}$ through $\hat{\Phi}_{\alpha}(l)$, and $\left(\frac{\partial}{\partial t_{1}^{(\alpha)}}\right)^{k} \hat{\Phi}^{\left\{U_{\alpha}\right\}}\left(l^{(\alpha)}\right)=\left(\frac{\partial}{\partial l^{(\alpha)}}\right)^{k} \hat{\Phi}^{\left\{U_{\alpha}\right\}}\left(l^{(\alpha)}\right)$ - through $\partial^{k} \hat{\Phi}_{\alpha}(l)$. Thus

$$
\begin{aligned}
& Z_{N_{1} \ldots N_{p-1}}^{A_{p-1}}\left\{t^{(1)} \ldots t^{(p-1)}\right\}= \\
& =\Delta\left(\frac{\partial}{\partial l^{(1)}}\right) \prod_{\alpha=1}^{p-2} \Delta\left(\frac{\partial}{\partial l^{(\alpha)}}, \frac{\partial}{\partial l^{(\alpha+1)}}\right) \Delta\left(\frac{\partial}{\partial l^{(p-1)}}\right) \times \\
& \times\left.\prod_{\alpha=1}^{p-1}\left(\frac{1}{N_{\alpha} !} \prod_{j=1}^{N_{\alpha}} \hat{\Phi}_{\alpha}\left(l_{j}^{(\alpha)}\right)\right)\right|_{l^{(\alpha)}=0} .
\end{aligned}
$$

If $p-1=1$, differential operator is just a square of determinant $\Delta(\partial / \partial l)$ and we can use the relation

$$
\begin{aligned}
\Delta^{2}(h) & =\sum_{P} \operatorname{Det}_{i j} h_{P(j)}^{i+j-2}= \\
& =\sum_{P} \operatorname{Det}\left[\begin{array}{cccc}
1 & h_{P(2)} & h_{P(3)}^{2} \ldots & h_{P\left(N_{1}\right)}^{N_{1}-1} \\
h_{P(1)} & h_{P(2)}^{2} & h_{P(3)}^{3} \ldots & h_{P\left(N_{1}\right)}^{N_{(}} \\
h_{P(1)}^{2} & h_{P(2)}^{3} & h_{P(3)}^{4} \ldots & h_{P\left(N_{1}\right)}^{N_{1}+1} \\
& & \ldots & \\
h_{P(1)}^{N_{1}-1} & h_{P(2)}^{N_{1}} & h_{P(3)}^{N_{1}+1} \ldots & h_{P\left(N_{1}\right)}^{2 N_{1}-2}
\end{array}\right]
\end{aligned}
$$


where the sum is over all the $N$ ! permutations $P$ of $N$ elements $1 \ldots N$, in order to conclude that (3.45) reproduces our old formula (3.33), (3.38): $Z_{N}=\operatorname{Det}_{i j} \partial^{i+j-2} \hat{\Phi}$.

For $p-1=2$ we need to use a more complicated analogue of (3.46):

$$
\begin{aligned}
& \Delta(h) \Delta\left(h, h^{\prime}\right) \Delta\left(h^{\prime}\right)= \\
& =\sum_{P} \sum_{P^{\prime}} \operatorname{Det}\left[\begin{array}{cccccc}
1 & h_{P(2)} \ldots & h_{P(1}^{N_{1}-1} & 1 & \bar{h}_{\bar{P}(2)} \ldots & \bar{h}_{\bar{P}\left(N_{2}\right)}^{N_{2}-1} \\
h_{P(1)} & h_{P(2)}^{2} \ldots & h_{P\left(N_{1}\right)}^{N_{1}} & \bar{h}_{\bar{P}(1)} & \bar{h}_{\bar{P}(2)}^{2} \ldots & \bar{h}_{\bar{P}\left(N_{2}\right)}^{N_{2}} \\
h_{P(1)}^{2} & h_{P(2)}^{3} \ldots & h_{P\left(N_{1}\right)}^{N N_{1}} & \bar{h}_{\bar{P}(1)}^{2} & \bar{h}_{\bar{P}(2)}^{3} \ldots & \bar{h}_{\bar{P}\left(N_{2}\right)}^{N_{2}+1} \\
h_{P(1)}^{\mathcal{N}_{1}-1} & h_{P(2)}^{\mathcal{N}_{1}} \ldots & h_{P\left(N_{1}\right)}^{\mathcal{N}+N_{1}-2} & \bar{h}_{\bar{P}(1)}^{\mathcal{N}_{2}-1} & \bar{h}_{\bar{P}(2)}^{\mathcal{N}_{2}} \ldots & \bar{h}_{P\left(N_{2}\right)}^{\mathcal{N}+N_{2}-2}
\end{array}\right]
\end{aligned}
$$

where $\mathcal{N}=\sum_{\alpha=1}^{p-1} N_{\alpha}$. Making use of this formula, we conclude that the r.h.s. of (3.45) for $p-1=2$ is also representable in the form of determinant:

$$
\operatorname{Det}\left[\begin{array}{cccccc}
\hat{\Phi} & \partial \hat{\Phi} \ldots & \partial^{N_{1}-1} \hat{\Phi} & \hat{\bar{\Phi}} & \partial \hat{\bar{\Phi}} \ldots & \partial^{N_{2}-1} \hat{\bar{\Phi}} \\
\partial \hat{\Phi} & \partial^{2} \hat{\Phi} \ldots & \partial^{N_{1}} \hat{\Phi} & \partial \hat{\bar{\Phi}} & \partial^{2} \hat{\bar{\Phi}} \ldots & \partial^{N_{2}} \hat{\bar{\Phi}} \\
\partial^{\mathcal{N}-1} \hat{\Phi} & \partial^{\mathcal{N}} \hat{\Phi} \ldots & \partial^{\mathcal{N}+N_{1}-2} \hat{\Phi} & \partial^{\mathcal{N}-1} \hat{\bar{\Phi}} & \partial^{\mathcal{N}} \hat{\bar{\Phi}} \ldots & \partial^{\mathcal{N}+N_{2}-2} \hat{\bar{\Phi}}
\end{array}\right]
$$

here $\hat{\Phi}=\hat{\Phi}_{1}, \hat{\bar{\Phi}}=\hat{\Phi}_{2}$ and all arguments $l^{(\alpha)}=0$. It is especially easy to check formula (3.47) in the simplest case of $N_{1}=N_{2}=1$. Then it just says that $\bar{h}-h=\operatorname{Det}\left[\begin{array}{ll}1 & 1 \\ h & \bar{h}\end{array}\right]$. Analogues expressions for $p-1>2$ are more involved, they are no longer just determinants: this is obvious already from consideration of the simplest case of $N_{1}=\ldots=N_{p-1}=1$, the product $\prod_{\alpha=1}^{p-2}\left(h^{(\alpha)}-h^{(\alpha+1)}\right)$ is no longer determinant of any nice matrix.

\subsection{Orthogonal polinomials}

Formalism of orthogonal polinomials was intensively used at the early days of the theory of matrix models. It is applicable to scalar-product eigenvalue models and allows to further transform (diagonalize) the remaining determinants into products. In variance with both reduction from original $N^{2}$-fold matrix integrals to the eigenvalue problem, which (when possible) reflects a physical phenomenon - decoupling of angular (unitary-matrix) degrees of 
freedom (associated with $d$-dimensional gauge bosons), - and with occurence of determinant formulas which reflects integrability of the model, orthogonal polinomials appear more as a technical device. Essentially orthogonal polinomials are necessary if wants to explicitly separate dependence on the the size $N$ of the matrix in the matrix integral ("zero-time") from dependencies on all other time-variables and to explicitly construct variables, which satisfy Toda-like equations. However, modern description of integrable hierarchies in terms of $\tau$-functions does not require explicit separation of the zero-time and treats it more or less on the equal fooring with all other variables, thus making the use of orthogonal polinomials unnecessary. Still this technique remains in the arsenal of the matrix model theory ${ }^{2}$ and we now briefly explain what it is about. At the end of this section two simple applications will be also described: one to evaluation of the volume of the unitary group, another - to direct proof of equivalence of the ordinary 1-matrix model and the Gaussian Kontsevich model. Both these examples make use of explicitly known orthogonal Hermite polinomials and in this sense are not quite representative: usually orthogonal polinomials are not known explicitly. Some applications of such "abstract" theory of orthogonal polinomials to the study of matrix models will be mentioned in the following sections.

In the context of the theory of scalar-product matrix models orthogonal polinomials naturally arise when one notes that after partition functions appears in a simple determinantal form of eq.(3.30), any linear change of basises $h^{i} \rightarrow Q_{i}(h)=\sum_{k} A_{i k} h_{k}, \bar{h}^{j} \rightarrow \bar{Q}_{j}(\bar{h})=\sum_{l} B_{j l} \bar{h}^{l}$ can be easily performed and $Z \longrightarrow Z \cdot \operatorname{det} A \cdot \operatorname{det} B$. In particular, if $A$ and $B$ are triangular with units at diagonals, their determinants are just unities and $Z$ does not change at all. This freedom is, however, enough, to diagonalize the scalar product and choose polinomials $Q_{i}$ and $\bar{Q}_{j}$ so that

$$
\left\langle Q_{i}(h) \mid \bar{Q}_{j}(\bar{h})\right\rangle=e^{\phi_{i}} \delta_{i j} .
$$

$Q_{i}$ and $\bar{Q}_{j}$ defined in this way up to normalization are called orthogonal polinomials. (Note that $\bar{Q}$ does not need to be a complex conjugate of $Q$ : "bar" does not mean complex conjugation.) Because of above restriction on

\footnotetext{
${ }^{21}$ Of course, one can also use this link just with the aim to put the rich and beautifull mathematical theory of orthogonal polinomials into the general context of string theory. Among interesting problems here is the matrix-model description of $q$-orthogonal polinomials.
} 
the form of matrices $A$ and $B$ these polinomials are normalized so that

$$
Q_{i}(h)=h^{i}+\ldots ; \quad \bar{Q}_{j}(\bar{h})=\bar{h}^{j}+\ldots
$$

i.e. the leading power enters with the unit coefficient. From (3.30) and (3.48) it follows that

$$
Z_{N}=\prod_{i=1}^{N} e^{\phi_{i-1}} .
$$

This formula is essentially the main outcome of orthogonal polinomials theory fro matrix models: it provides complete separation of the $N$-dependence of $Z$ (on the size of the matrix) from that on all other parameters (which specify the shape of potential, i.e. the measure $d \mu_{h, \bar{h}}$ ), this information is encoded in a rather complicated fashion in $\phi_{i}$. As was already mentioned, any feature of matrix model can be examined already at the level of eq.(3.30), which does not refer to orthogonal polinomials and thus they are not really relevant for the subject.

We can, however, reverse the problem and ask, what can matrix models provide for the theory of orthogonal polinomials.2 The first question to ask in the theory of orthogonal polinomials is:

Given the measure $d \mu_{h, \bar{h}}$, what are the corresponding orthogonal polinomials?

Usually the answer to this type of questions is not at all straightforward. Its complexity, however, depends on what one agrees to accept as a suitable answer. Of particular interest for our purposes below would be integral representations. It would be very helpfull to have just an integral transformation, converting the set of orthogonal polinomials for given $d \mu_{h, \bar{h}}$ into some standard set, like $Q_{i}^{(0)}=x^{i}$. Unfortunately, such transformation is rarely available, though there are important examples: classical orthogonal polinomials and their $q$-analogues (expressed through the $(q$-)hypergeometric functions, which usually possess integral representation of a simple form, see [69] for an introductory review of such integral formulas, which are in fact

\footnotetext{
${ }^{22}$ Of course, we can hardly get anything new for that theory, but the purpose is to see, which features are immediate consequences of the "physically-inspired" approach. As usually this can help to somehow organize the existing knowledge in appropriate system. This is, however, not our goal in these notes: only a very simple example will be mentioned, which will be also of use in our futher considerations.
} 
well known in CFT). The simplest example of this kind, which will be used below is the set of Hermite polinomials:

$$
\begin{aligned}
& \operatorname{He}_{k}(h)=\frac{1}{\sqrt{2 \pi}} e^{\frac{h^{2}}{2}} \int(i x)^{k} e^{-\frac{x^{2}}{2}-i x h} d x=\left(h-\frac{d}{d h}\right)^{k} \cdot 1= \\
& =e^{\frac{h^{2}}{2}}\left(-\frac{d}{d h}\right)^{k} e^{-\frac{h^{2}}{2}}=\frac{1}{2^{k}} e^{\frac{h^{2}}{4}}\left(h-2 \frac{d}{d h}\right)^{k} e^{-\frac{h^{2}}{4}}=h^{k}+\ldots,
\end{aligned}
$$

orthogonal with the local measure $d \mu_{h}=e^{-\frac{h^{2}}{2}}$.

For generic measure the answer of this type does not exist in any universal form. However, matrix models still provide a somewhat peculiar integral representation for any measure, with the number of integrations depending on the number of polinomial. In order to obtain this expression, let us consider a slight generalization of formula (3.27)

$$
Z_{N}\left\{\lambda_{\gamma}\right\} \equiv \frac{1}{N !} \prod_{k=1}^{N} \int d \mu_{h_{k}, \bar{h}_{k}} \Delta(h) \Delta(\bar{h}) \prod_{k, \gamma}\left(\lambda_{\gamma}-h_{k}\right)
$$

Then $\Delta(h) \prod_{k, \gamma}\left(\lambda_{\gamma}-h_{k}\right)=\Delta(h, \lambda) / \Delta(\lambda)$, and $\lambda_{\gamma}$ can be just considered as $h_{N+\gamma}$, which are not integrated over in (3.51). Then it is clear that

$$
\Delta(h, \lambda)=\operatorname{Det}\left(\begin{array}{ll}
Q_{i-1}\left(h_{k}\right) & Q_{N+\gamma-1}\left(h_{k}\right) \\
Q_{i-1}\left(\lambda_{\delta}\right) & Q_{N+\gamma-1}\left(\lambda_{\delta}\right)
\end{array}\right)
$$

while $\Delta(\bar{h})=\operatorname{Det}_{j k} \bar{Q}_{j-1}\left(\bar{h}_{k}\right)$. Since all the $Q_{N+\gamma-1}\left(h_{k}\right)$ are orthogonal to all $\bar{Q}_{j-1}\left(\bar{h}_{k}\right)$ (because $N+\gamma-1 \neq j-1$ ), we obtain:

$$
Z_{N}\left\{\lambda_{\delta}\right\}=\frac{\operatorname{det}_{\gamma \delta} Q_{N+\gamma-1}\left(\lambda_{\delta}\right)}{\Delta(\lambda)} Z_{N}
$$

In particular,

$$
Q_{N}(\lambda)=\frac{Z_{N}\{\lambda\}}{Z_{N}}
$$

where both the numerator and denominator can be represented by $N \times N$ matrix integrals.

Inverse "main question" of the theory of orthogonal polinomials is: 
Given a set of polinomials

$$
\begin{aligned}
& Q_{i}(h)=h_{i}+\ldots, \\
& \bar{Q}_{j}(\bar{h})=\bar{h}_{j}+\ldots,
\end{aligned}
$$

what is the measure $d \mu_{h, \bar{h}}$ w.r.to which they form an orthogonal system?

We shall not discuss the complete answer to this question and consider only the case of the local measure, when $\bar{Q}_{i}=Q_{i}$. Then usually the answer does not exist at all: not every system of polinomials is orthogonal w.r.to some local measure. It is easy to find the necessary (and in fact sufficient) condition. As was mentioned above, the local measure is distinguished by the property that multiplication by (any function of) $h$ is Hermitean operator:

$$
\langle h f(h) \mid g(\bar{h})\rangle=\langle f(h) \mid \bar{h} g(\bar{h})\rangle, \quad \text { if } d \mu_{h, \bar{h}} \sim \delta(h-\bar{h}) .
$$

This property implies, that the coefficients $c_{i j}$ in the recurrent relation

$$
h Q_{i}(h)=Q_{i+1}(h)+\sum_{j=0}^{i} c_{i j} Q_{j}(h)
$$

are almost all vanishing. Indeed: for $j<i$

$$
\begin{aligned}
c_{i j} & =\frac{\left\langle h Q_{i}(h) \mid Q_{j}(\bar{h})\right\rangle}{\left\langle Q_{j}(h) \mid Q_{j}(\bar{h})\right\rangle}=\frac{\left\langle Q_{i}(h) \mid \bar{h} Q_{j}(\bar{h})\right\rangle}{\left\langle Q_{j}(h) \mid Q_{j}(\bar{h})\right\rangle}= \\
& =\delta_{i, j+1} \frac{\left\langle Q_{i}(h) \mid Q_{i}(\bar{h})\right\rangle}{\left\langle Q_{j}(h) \mid Q_{j}(\bar{h})\right\rangle}=\delta_{j, i-1} e^{\phi_{i}-\phi_{i-1}} .
\end{aligned}
$$

In other words, polinomials, orthogonal w.r.to a local measure are obliged to satisfy the "3-term recurrent relation":

$$
h Q_{i}(h)=Q_{i+1}(h)+C_{i} Q_{i}(h)+R_{i} Q_{i-1}(h)
$$

(the coefficient in front of $Q_{i+1}$ can be of course changed by the change of normalization). Parameter $C_{i}$ vanishes if the measure is even (symmetric under the change $h \rightarrow-h$ ), then polinomials are split into two orthogonal subsets: even and odd in $h$. Partition function (3.49) of the one-component model can be expressed through parameters $R_{i}=e^{\phi_{i}-\phi_{i-1}}$ of the 3 -term relation:

$$
Z_{N}=Z_{1} \prod_{i=1}^{N-1} R_{i}^{N-i}
$$


thus defining a one-component matrix model (i.e. particular shape of potential), associated with any system of orthogonal polinomials.

Our "inverse main question" in the case of the local measure should be now formulated as follows: Given a set of orthogonal polinomials $Q_{i}(h)=$ $h^{i}+\ldots$ which satisfy the 3-term relation (3.58), what is the measure $d \mu_{h}$ ?

As every complete orthogonal system of functions, orthogonal polinomials satisfy the completeness relation:

$$
\sum_{i=0}^{\infty} e^{-\phi_{i}} \bar{Q}_{i}(\bar{h}) Q_{i}(h)=\delta^{\{d \mu\}}(\bar{h}, h),
$$

where $\delta$-function, associated with the measure $d \mu_{h, \bar{h}}$ is defined so that

$$
\iint f(h) \delta^{\{d \mu\}}\left(\bar{h}, h^{\prime}\right) d \mu_{h, \bar{h}}=f\left(h^{\prime}\right)
$$

for any function $f(h)$. Since for the local measure $d \mu_{h}=e^{U(h)} d h$ the $\delta$ function is just $\delta^{\{d \mu\}}(\bar{h}, h)=e^{-U(h)} \delta(\bar{h}-h)$, as an answer to our question we can take a representation of $U(h)$ in terms of the corresponding orthogonal polinomials:

$$
e^{-U(h)} \delta(\bar{h}-h)=\sum_{k=0}^{\infty} \frac{Q_{k}(\bar{h}) Q_{k}(h)}{\left\langle Q_{k} \mid Q_{k}\right\rangle} .
$$

As usually this relation should be understood as analytical continuation. The squared norms $\left\|Q_{k}\right\|^{2}$ in denominator are expressed through the coefficients $R_{i}$ of the 3 -term relation up to an overall constant: $\left\|Q_{k}\right\|^{2}=\prod_{i=1}^{k} R_{i} \|$ $Q_{0} \|^{2}$.

For example, in the case of Hermite polinomials (3.50) we have:

$$
\begin{aligned}
\operatorname{He}_{k+1}(h)=\left(h-\frac{d}{d h}\right) \mathrm{He}_{k}(h)= & h \mathrm{He}_{k}(h)-\frac{d}{d h} \operatorname{He}_{k}(h)= \\
& =h \mathrm{He}_{k}(h)-k \mathrm{He}_{k-1}(h)
\end{aligned}
$$

(the last equality holds because $\frac{d}{d h}$ and $h-\frac{d}{d h}$ play the role of annihilation and creation operators respectively). This means that the 3-term relation is satisfied with $R_{k}=k$ and thus $\left\|\mathrm{He}_{k}\right\|^{2}=\left\|\mathrm{He}_{0}\right\|^{2} k$ ! We shall use the 
normalization condition $\left\|H e_{0}\right\|^{2}=\sqrt{2 \pi}$. Then for $e^{-U(h)}$ we get:

$$
\begin{gathered}
e^{-U(h)} \delta(\bar{h}-h)= \\
\sum_{k=0}^{\infty} \frac{\operatorname{He}_{k}(\bar{h}) \operatorname{He}_{k}(h)}{\left\|H e_{k}\right\|^{2}}=\frac{1}{\sqrt{2 \pi}} \sum_{k=0}^{\infty} \frac{1}{k !}\left(h-\frac{d}{d h}\right)^{k}\left(\bar{h}-\frac{d}{d \bar{h}}\right)^{k} \cdot 1= \\
=\frac{1}{\sqrt{2 \pi}} e^{\frac{h^{2}}{2}+\frac{\bar{h}^{2}}{2}} \sum_{k=0}^{\infty} \frac{1}{k !}\left(\frac{d^{2}}{d h d \bar{h}}\right)^{k} e^{-\frac{h^{2}}{2}-\frac{\bar{h}^{2}}{2}}=\frac{1}{\sqrt{2 \pi}} e^{\frac{h^{2}}{2}+\frac{\bar{h}^{2}}{2}} e^{\frac{d^{2}}{d h d \bar{h}} e^{-\frac{h^{2}}{2}-\frac{\bar{h}^{2}}{2}}=} \\
=\frac{1}{\sqrt{2 \pi}} \operatorname{Im} \iint \frac{d \alpha d \bar{\alpha}}{2 \pi} e^{-\alpha \bar{\alpha}} e^{\frac{h^{2}}{2}+\frac{\bar{h}^{2}}{2}} e^{\alpha \frac{d}{d h}+\bar{\alpha} \frac{d}{d h}} e^{-\frac{h^{2}}{2}-\frac{\bar{h}^{2}}{2}}= \\
=\frac{1}{\sqrt{2 \pi}} \operatorname{Im} \iint \frac{d \alpha d \bar{\alpha}}{2 \pi} e^{-\frac{1}{2}(\alpha+\bar{\alpha})^{2}} e^{-\frac{1}{2}(\alpha+\bar{\alpha})(h+\bar{h})} e^{-\frac{1}{2}(\alpha-\bar{\alpha})(h-\bar{h})}= \\
=e^{\frac{h^{2}}{2}} \delta(h-\bar{h}) .
\end{gathered}
$$

\subsection{Scalar-product models in Miwa parametrization}

We shall now make the first step towards clarification of the interrelation between the scalar-product and Kontsevich models. We already know that in the latter case the important role is played by representation of timevariables in the form of

$$
T_{k}=\frac{1}{k} \operatorname{tr} \Lambda^{-k}
$$

with $n \times n$ matrix $\Lambda$, which will be further refered to as Miwa parametrization (expressions of some similar form were first introduced in [70]). Let us now perfrom such transformation in the case of the scalar-product model. Let us use eq.(3.31) to define the time-dependence of the measure, only ignore the $\bar{t}$-variables. Namely, introduce $d \mu_{h, \bar{h}}=e^{U(h)} d \hat{\nu}_{h, \bar{h}}$ (i.e. $d \hat{\nu}_{h, \bar{h}}=e^{\bar{U}(\bar{h})} d \hat{\mu}_{h, \bar{h}}$ ). Substitute

$$
t_{k}=\mp\left(\frac{1}{k} \operatorname{tr} \Lambda^{-k}+r_{k}\right)
$$

and obtain:

$$
\begin{aligned}
e^{U(h)}=e^{-\hat{V}(h)} e^{\mp \operatorname{tr} \sum_{k=1}^{\infty} \frac{1}{k}\left(\frac{h}{\Lambda}\right)^{k}}=e^{-\hat{V}(h)} \frac{\operatorname{det}^{ \pm 1}(\Lambda-h \cdot I)}{\operatorname{det} \Lambda}= \\
=\frac{e^{-\hat{V}(h)}}{\operatorname{det} \Lambda} \prod_{\gamma=1}^{n}\left(\lambda_{\gamma}-h\right)^{ \pm 1}
\end{aligned}
$$


where $\hat{V}(h) \equiv \pm \sum_{k} r_{k} h^{k}$. Let us choose upper signs in these formulas. Then we can use eqs.(3.51) and (3.53) to conclude that in Miwa parametrization

$$
Z_{N}^{\{d \mu\}}=\frac{1}{(\operatorname{det} \Lambda)^{N}} Z_{N}^{\{d \hat{\nu}\}}\left\{\lambda_{\delta}\right\}=Z_{N}^{\{d \hat{\nu}\}} \frac{\operatorname{det}_{\gamma \delta} \hat{Q}_{N+\gamma-1}\left(\lambda_{\delta}\right)}{\Delta(\lambda)(\operatorname{det} \Lambda)^{N}}
$$

where $d \hat{\nu}_{h, \bar{h}} \equiv e^{-\hat{V}(\bar{h})} d \nu_{h, \bar{h}}$ and $\hat{Q}_{k}$ are the corresponding orthogonal polinomials. In other words, we reduced the model with potential $U(h)$ to another model with potential, $-\hat{V}(h)$, and expressed the difference in terms of orthogonal polinomials $\hat{Q}_{k}$ :

$$
\frac{Z_{N}^{\{d \mu\}}}{Z_{N}^{\{d \hat{\nu}\}}}=\frac{1}{(\operatorname{det} \Lambda)^{N}} \cdot \frac{\operatorname{det}_{\gamma \delta} \hat{Q}_{N+\gamma-1}\left(\lambda_{\delta}\right)}{\Delta(\lambda)} .
$$

If $\hat{V}(h)$ is adjusted to give rise to some simple orthogonal polinomials, (i.e. if the new model $Z_{N}^{\{d \hat{\nu}\}}$ is easy to solve), this representation can considerably simplify the original model.

Another interpretation of this formula is that we obtained a GKM-like representation of the from of (3.19) for the discrete scalar-product model. The only difference is that $\varphi_{\gamma}^{\{V\}}$ in (3.19) are changed for $\hat{Q}_{\gamma-1}$ in (3.67). This is an important difference, because $\varphi_{\gamma}^{\{V\}}$ in GKM are defined to by integral formulas like (3.15), $\varphi_{\gamma}^{\{V\}}=\left\langle\left\langle x^{\gamma-1}\right\rangle\right\rangle$ or, alternatively, satisfy the recursive relations like (3.21). Moreover, generic $\varphi_{\gamma}^{\{V\}}$ are infinite formal series in $\lambda^{-1}$, while $Q_{\gamma-1}$ are orthogonal polinomials. This discreapancy is one of important stimuli for further developement of the concept of Generalized Kontsevich model, as well as for search for convenient integral representations for orthogonal polinomials.

There is, however, at least one interesting situation when the two formulas indeed coincide. This is the case of Gaussian potentials $V$ and $\hat{V}$, when both $\varphi_{\gamma}^{\{V\}}$ and $Q_{\gamma-1}$ are represented by orthogonal Hermite polinomials, which possess integral representation, exactly adequate in the context of GKM. This is the subject of our consideration in the next subsection.

\subsection{Equivalence of the discrete 1-matrix and Gaussian Kontsevich models}

Let us take the ordinary 1-matrix model with the local measure $d \mu_{h}=$ $e^{U(h)} d h$ to be the scalar-product model, considered in the previous subsection 
and take Miwa parametrization with upper signs and with $r_{k}=-\frac{1}{2} \delta_{k, 2}$ (as we did in the section 2.6). Then $\hat{V}(h)=\sum_{k} r_{k} h^{k}=-\frac{h^{2}}{2}=\frac{(i h)^{2}}{2}$. The relevant orthogonal polinomials $\hat{Q}$ are just Hermite polinomials of imaginary argument:3 $Q_{k}^{\left\{-\frac{h^{2}}{2} d h\right\}}=i^{-k} \mathrm{He}_{k}(i h)=h^{k}+\ldots$ These polinomials possess an integral representation (3.50):

$$
i^{1-k} \mathrm{He}_{k-1}(i h)=\frac{1}{\sqrt{2 \pi}} e^{-\frac{h^{2}}{2}} \int x^{k-1} e^{-\frac{x^{2}}{2}+x h} d x \stackrel{(3.20)}{=} \varphi_{k}^{\left\{\frac{x^{2}}{2}\right\}}(h) .
$$

Using (3.67) and (3.19) we obtain a remarkable relation between the two matrix models:

$$
\begin{gathered}
\frac{Z_{N}\left\{t_{0}=0 ; t_{k}=-\frac{1}{k} \operatorname{tr} \Lambda^{-k}+\frac{1}{2} \delta_{k, 2}\right\}}{Z_{N}\left\{t_{k}=\frac{1}{2} \delta_{k, 2}\right\}}=\frac{\int_{N \times N} d H e^{\sum_{k=0}^{\infty} t_{k} \operatorname{Tr} H^{k}}}{\int_{N \times N} d H e^{\frac{1}{2} H^{2}}}= \\
=\frac{e^{-\operatorname{tr} \frac{\Lambda^{2}}{2}}}{(2 \pi)^{\frac{n^{2}}{2}}(\operatorname{det} \Lambda)^{N}} \int_{n \times n} d X(\operatorname{det} X)^{N} e^{-\operatorname{tr} \frac{X^{2}}{2}+\Lambda X}=\mathcal{Z}_{\frac{X^{2}}{2}}\{N, t\},
\end{gathered}
$$

where $Z_{N}\left\{t_{k}=\frac{1}{2} \delta_{k, 2}\right\}=(-2 \pi)^{\frac{N^{2}}{2}} c_{N}$. This relation can be also regarded as an identity

$$
\begin{array}{r}
\frac{\int_{N \times N} d H e^{\frac{1}{2} \operatorname{Tr} H^{2}} \operatorname{Det}(\Lambda \otimes I-I \otimes H)}{\int_{N \times N} d H e^{\frac{1}{2} \operatorname{Tr} H^{2}}}= \\
=\frac{\int_{n \times n} d X e^{-\frac{1}{2} \operatorname{tr} X^{2}} \operatorname{det}^{N}(X+\Lambda)}{\int_{n \times n} d X e^{-\frac{1}{2} \operatorname{tr} X^{2}}},
\end{array}
$$

valid for any $\Lambda$. Note that integrals are of differents sizes: $N \times N$ at the l.h.s. and $n \times n$ at the r.h.s. While $N$-dependence is explicit at both sides of the equation, the $n$-dependence at the l.h.s. enters only implicitly: through the allowed domain of variation of variables $t_{k}=-\frac{1}{k} \operatorname{tr} \Lambda^{-k}+\frac{1}{2} \delta_{k, 2}$. (This can serve as an illustration to the general statement that the shape of Kontsevich

\footnotetext{
${ }^{23}$ Note that this system of functions $\varphi_{k}=i^{-k} \mathrm{He}_{k}(i h)$ looks like $\varphi_{0}=1, \varphi_{1}=h, \varphi_{2}=$ $h^{2}+1, \ldots$, and does not resemble any set of orthogonal polinomials with a local measure (for example the product $\varphi_{0} \cdot \varphi_{2}=h^{2}+1$ may seem positive definite, this being inconsistent with orthogonality requirement $\left\langle\varphi_{0} \mid \varphi_{2}\right\rangle=0$ ). The thing is that integration at the l.h.s. of eq.(3.69) is well defined only along the imaginary axis, while integrals along the real axis are understood as analytical continuation.
} 
partition function $\mathcal{Z}_{V}$, considered as a function of $T$ 's rather than $L$ or $\Lambda$, is independent of the matrix size $n$.) Identity (3.69) was anticipated from the study of Ward identites for the Gaussian Kontsevich model in [56] (see eq. (2.53) in the section 2.6 above), and it was derived in the present form in ref. 36.

Eq.(3.69) can be used to perfrom analytical continuation in $N$ and define what is $Z_{N}$ for $N$, which are not positive integers. Since $c_{N}=0$ for all negative integers (see eq.(3.77) below), the same is true for $Z_{N}$. In the next section 4 we shall see that it is characteristic property of $\tau$-functions of forced hierarchies.

\subsection{Volume of unitary group}

Formalism of orthogonal polinomials provides also a simple derivation of eq. (3.6) for the volume of unitary group. Consider eq.(3.4) with $U(H)=H^{2}$. Then Gaussian matrix integral can be easily evaluated:

$$
c_{N} \int_{N \times N} d H e^{-\frac{1}{2} \operatorname{Tr} H^{2}}=c_{N} \prod_{i=1}^{N} \int d H_{i i} e^{-\frac{1}{2} H_{i i}^{2}} \prod_{i<j}^{N} \int d^{2} H_{i j} e^{-\left|H_{i j}\right|^{2}}=(2 \pi)^{N^{2} / 2},
$$

while according to eqs.(3.48) and (3.49) the same integral is equal to

$$
\frac{1}{N !} \prod_{i=1}^{N} \int_{-\infty}^{+\infty} e^{-\frac{1}{2} h_{i}^{2}} \prod_{i>j}^{N}\left(h_{i}-h_{j}\right)^{2}=\prod_{j=1}^{N}\left\|\mathrm{He}_{j-1}\right\|^{2}
$$

Here $\left\|\mathrm{He}_{j-1}\right\|$ stand for the norms of orthogonal Hermite polinomials (3.50), $\left\|\mathrm{He}_{k}\right\|^{2}=\sqrt{2 \pi} k$ !. Comparing the two expressions for the same integral we get:

$$
c_{N}^{-1}=(2 \pi)^{\frac{N^{2}}{2}} \prod_{k=0}^{N-1} \frac{1}{\sqrt{2 \pi} k !}=\frac{(2 \pi)^{\frac{N(N-1)}{2}}}{\prod_{k=0}^{N-1} k !} .
$$

According to (3.5)

$$
c_{N}^{-1}=N ! \frac{\mathrm{Vol}_{U(N)}}{\left(\mathrm{Vol}_{U(1)}\right)^{N}}
$$


and $\mathrm{Vol}_{U(1)}=2 \pi$. Thus we obtain eq.(3.6):

$$
\operatorname{Vol}_{U(N)}=\frac{(2 \pi)^{\frac{N(N+1)}{2}}}{\prod_{k=0}^{N} k !}
$$

An example of somewhat more sophisticated (quantum) group-theoretical quantity, arising from Gaussian matrix models, is provided by the following formula for the $q$-factorial [71] (see also [72]):

$$
\frac{1}{(q, q)_{N}} \equiv \prod_{n=1}^{N} \frac{1}{1-q^{n}}=\frac{\iint_{N \times N} d H[d U] e^{-m^{2} \operatorname{Tr} H^{2}+\operatorname{Tr} H U H U^{\dagger}}}{\operatorname{Vol}_{U(N)} \int_{N \times N} d H e^{-m^{2} \operatorname{Tr} H^{2}}}
$$

Integral in the numerator is over Hermitean $(H)$ and unitary $(U) N \times N$ matrices, and $q \equiv m^{2}-\sqrt{m^{4}-1}$.

Explicit expression (3.71) can be used to prove that $c_{N}=0$ for all negative integer $N$ [36]. Eq. 3.71) defines $c_{N}$ only for positive integer $N$ as a finite product. There is an obvious prescription for analytical continuation of such products, provided continuation of the items is known (it can be considered as implied by the similar formula for integrals with the varying upper limit): Let

$$
F(N)=\sum_{k=-\infty}^{N} f(k)
$$

Then

$$
S(N) \equiv \sum_{k=1}^{N} f(k)=F(N)-F(0)
$$

and, obviously, $F(0)-F(-N)=\sum_{k=1-N}^{0} f(k)$, so that

$$
S(-N) \equiv F(-N)-F(0)=-\sum_{k=0}^{N-1} f(-k) .
$$

Exponentiation of this formula gives the rule for the products. In the case of $c_{N}$ one can treat factorials in (3.71) as Gamma-functions,

$$
(2 \pi)^{\frac{N(N-1)}{2}} c_{N}=\prod_{k=1}^{N} \Gamma(k)
$$


and obtain:

$$
(2 \pi)^{-\frac{N(N+1)}{2}} c_{-N}=\left(\prod_{k=0}^{N-1} \Gamma(-k)\right)^{-1}=0,
$$

because of the poles of $\Gamma$-functions.

\section{Integrable structure of eigenvalue models}

\subsection{The concept of integrability}

Integrable structure of dynamical system implies that all the dynamical characteristics - solutions of equations of motion for a classical system and functional integrals for a quantum one - can be found exactly. According to this description the notion of integrability is not very concrete, and in fact it evolves with time, including more and more classes of theories into the class of integable systems. Nowadays we consider the following types of theories as clearly belonging to this class:

- Free motion (classical or quantum) on group manifolds and homogeneous spaces;

- 2-dimensional conformal theories and their "integrable massive deformations";

- Integrable hierarchies of the (multicomponent) KP and Toda type and their reductions;

- Functional integrals, subjected to conditions of (generalized) DuistermaatHeckman theorem;

- (Eigenvalue) matrix models;

- Topological theories;

- Many supersymmetric models (at least those allowing for Nicolai transformation and/or Duistermaat-Heckman-like description);

- Systems with (infinitely) many local integrals of motion.

This list (nothing to say about the order of items) is rather arbitrary. Also different items are not really different and (as it should be) can be considered as different descriptions of the same reality. Now we discuss very briefly at least some of the most important views on the concept of integrability. 
Often the notion of integrability is related to occurence of "many enough" integrals of motion ("many enough" means equal to the number of degrees of freedom). This is, however, not such a rigid definition as one can think. In fact, in classical mechanics there is usually a complete set of integrals of motion available: just initial conditions in the phase space (or, to be more sophisticated, angle-action variables). The problem is, however, that

a) these obvious integrals are very complicated (non-local and multivalued) functionals of the current coordinates, and

b) in general situation they are very "unstable" under a small change of current coordinates ("divergency of trajectories").

In order to avoid these problems one usually imposes a "locality" condition on equations of motion. While this is a reasonable thing to do for particular classes of theories (e.g. possessing a well defined kinetic term, which is quadratic in momenta), this is not a nice decription in general situation, since "locality" is not invariant under arbitrary (including non-local) change of variables. In practice, when approached from this side, integrability implies a kind of "regular" behaviour of trajectories and some more or less nicel defined transformation from "natural" (or, better to say, "original") coordinates to the action-angle variables.

Situation becomes even less clear when quantum theory is considered, since "chaotic behaviour" no longer implies anything really "chaotic" for the quantum system. Again, very much depends on what kind of observables one wants to consider, and any notion of "regularity" is not enough under arbitrary change of variables.

This can be made even more transparent, if one recalls the idea of universality classes, so important in the modern theory. The idea is that even in the cases when behaviour of the system seems absolutely haotic from any naive point of view (like in the cases of turbulence or quantum gravity), one can and should introduce new variables (which can be very complicated functions of original ones), which have smooth and well defined correlation functions. In most cases one is not attempting to find a complete set of such variables (and thus some information is lost), but this reflects nothing but the current state of knowledge, and in fact in studies of $2 d$ quantum gravity the goal of complete descrption is already clearly fromulated.

Despite these comments, the "definition" of integrability in terms of "many enough" local integrals of motion should be put at the first place in our discussion, because most of the systems which were so far considered 
as integrable more or less naturally get into this class, allowing for some prefered choice of dynamical variables ("more or less" appears because some "minor" non-locality is usually present in any interesting examples, where angle-action variables are not obvious from the very beginning).

This "definition" is so unclear because we attempted to look for a generic description of integrability. Most interesting approaches, however, go from another direction. One starts from some simple system and then perform a change of variables, which makes it looking much more complicated (being still simple in its essense). This appears to be a much more fruitfull view on the problem and in fact all the other items of our list above are describable in this kind of terms.

A trivial, but surprisingly representative example of this approach is provided by a free particle, moving in flat $D$-dimensional space. The eigenfunctions of Laplace operator are just plain waves or, equivalently, spherical harmonics. The radial part of the $j$-th harmonic is already a not very simple function, satisfying the equation

$$
\left(-\frac{d^{2}}{d r^{2}}+\frac{D-1}{r} \frac{d}{d r}+\frac{C_{2}(j)}{r^{2}}\right) \psi(r)=E \psi(r) .
$$

This equation is of course less trivial than the original Laplace equation, but their solutions are related in a simple way. In order to find a solution of (4.1), say, for $j=0$, one should just take an angular average of a plane wave:

$$
\phi_{k}(r)=\int e^{i k \vec{r} \vec{\nu}} d^{D-1} \vec{\nu} ; \quad|\vec{\nu}|=1 .
$$

This integral representation expresses the solutions of (4.1) through Bessel functions, and this is in fact the proper way to derive the well-known formula:

$$
\phi_{k}(r)=2^{\frac{D}{2}-1} \Gamma\left(\frac{D}{2}\right)(k r)^{1-\frac{D}{2}} J_{\frac{D}{2}-1}(k r) .
$$

If one expands the exponent in the integral in a series, the standard expansion for the Bessel function arises.

A slightly more involved example is the quantum mechanical model of a particle in the potential $e^{-q}$, i.e. the theory of equation

$$
\left(-\frac{d^{2}}{d q^{2}}+e^{-q}\right) \psi(q)=0
$$


(one of course recognizes a simplified version of Toda models). It can be solved by projection of the simple Sshrödinger equation for a particle, moving on the upper part of the hyperboloid $x_{0}^{2}-x_{1}^{2}-x_{2}^{2}=1 ; x_{0}>0$ [73]. If

$$
x_{0}=\cosh \frac{q}{2}+\frac{1}{2} z^{2} e^{q / 2} ; \quad x_{1}=\sinh \frac{q}{2}-\frac{1}{2} z^{2} e^{q / 2} ; \quad x_{2}=z e^{q / 2},
$$

then $q=\log \left(x_{0}+x_{1}\right)$, Laplace operator on hyperboloid is

$$
L=\frac{\partial^{2}}{\partial q^{2}}-\frac{1}{2} \frac{\partial}{\partial q}+\frac{1}{4} e^{-q} \frac{\partial^{2}}{\partial z^{2}}
$$

and average of the wave function $\psi_{\lambda}(q, z)$ provides the following expression for solutions of (4.4):

$$
\psi_{\lambda}(q)=e^{i \lambda q} \int_{0}^{\infty} t^{2 i \lambda-1} e^{-\left(t+e^{q} / t\right)} d t .
$$

This idea, which is sometimes refered to as "projection method" (see 73 for a broad review), reveals hidden symmetries of some complicated systems (which do not possess any symmetry at all in the usual, Noether-like, sence of the word), by considering them as embedded into wider theories with more degrees of freedom. Quantum mechanical examples of applicability of the method are by no means exhausted by the two systems above, one can consider various projections, starting from (exactly solvable problem of) the free motion on any group manifold, and in general this gives rise to a very important theory of "zonal spherical functions", which nowadays is increasingly attracting attention because of its obvious links to integrability theory and quantum geometry (see [74 for discussion of the latter relation and [75], where also relations with orthogonal polinomials and Generalized Kontsevich model are partly revealed). An extremely important example of free motion on a group manifold (in the infinite-dimensional - Kac-Moody - case) is provided by 2-dimensional WZNW (Wess-Zumino-Novikov-Witten) model and the corresponding version of projection method is known as Hamiltonian reductions in conformal field theory. Again the resulting theories (like minimal conformal models) do not possess any kind of symmetry in the usual sense of the word, but still they are very simple and exactly solvable, remembering their origin in the theory of free fields.

In principle the theory which is reduced, i.e. complemented by constraints (initial conditions), does not need to be absolutely symmetric, i.e. to have 
Casimir operator or even zero (as in the WZNW case) as its Hamiltonian. It can be in fact possible to use the projection method to gain a lot of information about reductions of theories with more sophisticated Hamiltonians, which are non-trivial functions of group generators. The simplest example is provided by the theory of quantum-mechanical "quasi-exactly-solvable models" [76, 777 and its CFT-generalizations [77, [78]. A more elaborated technique has the name of "localization theory", (known also as geometrical quantization, Fourier analysis on group manifolds and Duistermaat-Heckman theory), it provides a very wide generalization of the above averaging procedure, which maped plain waves into Bessel functions. The classical sample system to illustrate all the aspects of integrability, starting from free motion and ending with anyonic statistics, $W_{\infty}$-algebras and $2 d$ Yang-Mills theory, is the Calogero-Sutherland system, which can be associated in a uniform way with any simple Lie algebras and in an "intermediately involved" form looks like a multiparticle theory in $1+1$ dimension with interaction potential $g^{2} \sin ^{-2} \epsilon\left(x_{i}-x_{j}\right)$. (See [73] for the introduction to the theory of Calogerotype models and [80, [81] for the new developements.)

All this discussion was necessary just to illustrate a very simple idea: the theory of free particles, though trivial, is in fact unexhaustively deep. It is enough to impose sophisticated initial conditions or perform a sophisticated change of variables in order to obtain very complicated dynamical systems, which after they are studied per se appear to be surprisingly system, the reason for this simplicity being that the real underlying dynamics is just trivial - that of the free particles,- though it may be very hard probelm to reveal this simplicity when the system is given. It is advantage of the general theory, that one can begin from the proper side: just from the theory of free particles and just start making it more and more complicated, by introducing different kind of variables, considering correlators of sophisticated operators and so on. Everything what can be obtained in this way is by definition trivially integrable, though it may be not so simple to guess for somebody who did not know where the particular system at the end of this procedure appeared from.

We now proceed to discussion of particularly important realization of this

${ }^{24}$ For various views and approaches to this theory see [61, 62, 63, [5], 64, 65, 64, 63], 44, 79. (So far there are no connections with Andersson localization in the solid state physics.) 
idea: the theory of $\bar{\partial}$-operators in $1_{C}$ dimension (i.e. the theory of free holomorphic fields in 2 real dimensions). When considered as functions of moduli of the bundles over Riemann surfaces (i.e. of boundary conditions, imposed on $2 d$ free fields), these simple objects (known as " $\tau=$ functions") start looking a little involved and after all appear related to sophisticated non-linear equations (but of course integrable) in 2 and 3 dimensions (like $\mathrm{KdV}$ or Kadomtsev-Petvishvili (KP) equation). We do not attempt to present an exhaustive theory of $\tau$-functions and integrable hierarchies (besides being still uncompleted, this is a very big field), but instead concentrate on the very core of it, which is just simple determinant formulas for the simplest $\tau$-functions (namely, associated with free-fermion theory and level $k=1$ Kac-Moody algebras). This issue will be discussed in some details, because besides being the basis of integrable hierarchies theory, it is also exactly the place, where the links with the matrix models are found.

\subsection{The notion of $\tau$-function}

There are several different definitions of $\tau$-functions, but all of them are particular realizations of the following idea: $\tau$-function is a generating functional of all the correlation functions in the theory of free particles in $1+1$ dimensions. This basic quantity is a kind of "det D", where " $D$ " is a timeevolution operator (continuous or discrete) and "det" is a sort of a product over eigenvalues of " $D$ ", which is usually expressed in the form of a functional integral, associated with free particles (it is not a priori Gaussian in original variables). This quantity is the most general definition of $\tau$-function.

In practice one is usually more specific. The mostly well studied version of $\tau$-function arises if one thinks about free particles of a peculiar type: free fermions with quadratic Hamiltonian and continuous time evolution, i.e. one considers a theory of spin- $1 / 2 b, c$-system (fermions) $\tilde{\psi}(\bar{z}, z), \psi(\bar{z}, z)$, described by the functional integral

$$
\begin{gathered}
\tau\{A\} \sim \operatorname{Det}(\bar{\partial}+\mathcal{A}) \sim \\
\sim \int D \tilde{\psi} D \psi \exp \left(\int_{d^{2} z} \tilde{\psi} \bar{\partial} \psi\right) \exp \left(\int_{d^{2} z} \int_{d^{2} \tilde{z}} A(z, \tilde{z}) \delta(\overline{\tilde{z}}-\bar{z}) \psi(z) \tilde{\psi}(\tilde{z})\right)
\end{gathered}
$$

where $\bar{z}$ plays the role of time and $\mathcal{A}=A(z, \tilde{z}) \delta(\overline{\tilde{z}}-\bar{z}) d \overline{\tilde{z}} d \bar{z}$ is some $\left(\frac{1}{2}, 1 ; \frac{1}{2}, 1\right)$ bidifferential (i.e. contains a factor of $d \tilde{z}^{1 / 2} d \overline{\tilde{z}} d z^{1 / 2} d \bar{z}$ ). 
Of course, one can think about more general $\tau$-functions, involving many fermions (this is often done), and more general $b, c$ - and $\beta, \gamma$-systems, in particular, arising in the context of WZNW model, associated to any KacMoody algebra of any level. ${ }^{25}$ Also of interset is consideration of discrete time evolution (described by difference rather than differential equations), though, as usual in the $2 d$ theories, this is not a really independent problem.

In the language of matrix models the restriction to free-fermion $\tau$ functions is essentially equivalent to restriction to eigenvalue models. Serious consideration of non-eigenvalue models, aimed at revealing their integrable (solvable) structure will certainly involve the theory of generic $\tau$-functions, but both these things are matters of the future research, and we"ll not go into details about them in these notes.

\section{$4.3 \tau$-function, associated with the free fermions}

Because of specific form of the Lagrangian in (4.7) the functional integral can be easily represented in Hamiltonian form, provided topology of the 2-surface on which $\bar{z}, z$ are coordinates, is trivial (genus 0: sphere or annulus). Namely, consider $\tilde{\psi}$ and $\psi$ as operator-valued functions of $z$ only (not of the time $\bar{z}$ ). Then the only reminiscent of kinetic term $\int_{d^{2} z} \tilde{\psi} \bar{\partial} \psi$ is canonical commutation relation:

$$
[\tilde{\psi}(\tilde{z}), \psi(z)]_{+}=\delta(\tilde{z}-z) d \tilde{z}^{1 / 2} d z^{1 / 2}
$$

Then

$$
\tau\{A\} \sim\left\langle 0\left|\exp \left(\oint_{d \tilde{z}} \oint_{d z} A(z, \tilde{z}) \psi(z) \tilde{\psi}(\tilde{z})\right)\right| 0\right\rangle
$$

Now it is usual to expand around $z=0$ :

$$
\psi(z)=\sum_{n \in Z} \psi_{n} z^{n} d z^{1 / 2} ; \quad \tilde{\psi}(z)=\sum_{n \in Z} \tilde{\psi}_{n} z^{-n-1} d z^{1 / 2}
$$

\footnotetext{
${ }^{25}$ The main technical difference between generic and "free-fermion" cases is that Lagrangian of generic free field theory is not just quadratic in scalar fields $\phi$, but can also contain particular combinations of exponents $e^{\phi}$. It also deserves noting that the most general expresiion, quadratic in scalar fields, if rewritten in terms of fermions is in fact quartic (but of course not a generic quartic interaction arises in this way). Integrable nature of certain quartic-fermion interactions is well known from the theory of Thirring models (in this class of models interactions are usually local).
} 


$$
\begin{aligned}
& {\left[\tilde{\psi}_{m}, \psi_{n}\right]_{+}=\delta_{m, n}} \\
& \psi_{m}|0\rangle=0 \text { for } m<0 ; \quad \tilde{\psi}_{m}|0\rangle=0 \text { for } m \geq 0 \\
& A(z, \tilde{z})=\sum_{m, n \in Z} z^{-m-1} \tilde{z}^{n} A_{m n} d z^{1 / 2} d \tilde{z}^{1 / 2}
\end{aligned}
$$

so that

$$
\oint_{d \tilde{z}} \oint_{d z} A(z, \tilde{z}) \psi(z) \tilde{\psi}(\tilde{z})=\sum_{m, n \in Z} A_{m n} \psi_{m} \tilde{\psi}_{n}
$$

In fact this expansion could be around any point $z_{0}$ and on a 2-surface of any topology: topological effects can be easily included as specific shifts of the functional $A(z, \tilde{z})$ - by combinations of the "hadle-gluing operators". Analogous shifts can imitate the change of basic functions $z^{n}$ for $z^{n+\alpha}$ and more complicated expressions (holomorphic 1/2-differentials with various boundary conditions on surfaces of various topologies).

One can now wonder, whether local functionals $A(z, \tilde{z})=U(z) \delta(\tilde{z}-$ $z) d z^{1 / 2} d \tilde{z}^{1 / 2}$ play any special role. The corresponding contribution to the Hamiltonian looks like ${ }^{20}$

$$
H_{\text {Cartan }}=\oint_{d z} U(z) \psi(z) \tilde{\psi}(z)=\oint_{d z} U(z) J(z),
$$

where

$$
J(z)=\psi(z) \tilde{\psi}(z)=\sum_{n \in Z} J_{n} z^{-n-1} d z
$$

is the $U(1)_{k=1}$ Kac-Moody current;

$$
J_{n}=\sum_{m \in Z} \psi_{m} \tilde{\psi}_{m+n} ; \quad\left[J_{m}, J_{n}\right]=m \delta_{m+n, 0}
$$

If scalar function (potential) $U(z)$ is expanded as $U(z)=\sum_{k \in Z} t_{k} z^{k}$, then

$$
H_{\text {Cartan }}=\sum_{n \in Z} t_{k} J_{k}
$$

\footnotetext{
${ }^{26}$ Note that normalization factor here is different by a factor of $\frac{1}{\sqrt{2}}$ from that in discussion of discrete models in sections 2.3, 2.7 and 2.8. This is not just a change of notations, since Miwa transformation can lead to different results when this normalization is changed. See a footnote in section 4.6 below for more detailed discussion.
} 
This contribution to the whole Hamiltonian can be considered distinguished for the following reason. Let us return to original expression (4.8) and try to consider it as a generating functional for all the correlation functions of $\tilde{\psi}$ and $\psi$. Naively, variation w.r.to $A(z, \tilde{z})$ should produce bilinear combination $\psi(z) \tilde{\psi}(\tilde{z})$ and this would solve the problem. However, things are not just so trivial, because operators involved do not commute (and in particular, the exponential operator in (4.8) should still be defined less symbolically, see next subsection). Things would be much simpler, if we can consider commuting set of operators: this is where abelian $U \hat{(1})_{k=1}$ subgroup of the entire $G L(\infty)_{k=1}$ (and even its purely commuting Borel subalgebra) enters the game. Remarkably, it is sufficient to deal with this abelian subgroup in order to reproduce all the correlation functions. ${ }^{27}$ The crucial point is the identity for free fermions (generalizable to any $b, c$-systems):

$$
: \psi(\lambda) \tilde{\psi}(\tilde{\lambda}):=: \exp \left(\int_{\lambda}^{\tilde{\lambda}} J\right):
$$

which is widely known in the form of bosonization formulas:28 if $J(z)=$ $\partial \phi(z)$,

$$
\begin{array}{cc}
\tilde{\psi}(\tilde{\lambda}) \sim: e^{\phi(\tilde{\lambda})}: \quad\left(: \psi(\infty) \tilde{\psi}(\tilde{\lambda}):=: e^{(\phi(\tilde{\lambda})-\phi(\infty))}:\right) \\
\psi(\lambda) \sim: e^{-\phi(\lambda)}: \quad\left(: \psi(\lambda) \tilde{\psi}(\infty):=: e^{(\phi(\infty)-\phi(\lambda))}:\right)
\end{array}
$$

This identity implies that one can generate any bilinear combinations of $\psi$ operators by variation of potential $U(z)$ only, moreover this variation should

27 We once again emphasize that this trick is specific for the free fermions and for the level $k=1$ Kac-Moody algebras, which can be expressed entirely in terms of free fields, associated with Cartan generators (modulo some unpleasant details, related to "cocycle factors" in the Frenkel-Kac representations [47], which are in fact reminiscents of free fields associated with the non-Cartan generators (parafermions) [58], - but can, however, be put under the carpet or/and taken into account "by hands" as "unpleasant but non-essential(?) sophistications).

28 Formulas in brackets are indeed correct, before them the usual symbolic relations are written. Using these formulas we get:

$$
: \psi(\lambda) \tilde{\psi}(\tilde{\lambda}):=: e^{\phi(\tilde{\lambda})-\phi(\lambda)}:=: e^{\int_{\lambda}^{\tilde{\lambda}} \partial \phi}:=: e^{\int_{\lambda}^{\tilde{\lambda}} J}:
$$

This identity can be of course obtained within fermionic theory, one should only take into account that $\psi$-operators are nilpotent, so that exponent of a single $\psi$-operator would be just a sum of two terms (polinomial). 
be of specific form:

$$
\begin{array}{r}
\Delta \oint U J=\Delta\left(\sum_{k \in Z} t_{k} J_{k}\right)=\int_{z}^{\tilde{z}} J=\sum_{k \in Z} \int_{z}^{\tilde{z}} z^{-k-1} d z= \\
=\sum_{k \in Z} \frac{1}{k} J_{k}\left(\frac{1}{z^{k}}-\frac{1}{\tilde{z}^{k}}\right)
\end{array}
$$

i.e.

$$
\Delta t_{k}=\frac{1}{k}\left(\frac{1}{z^{k}}-\frac{1}{\tilde{z}^{k}}\right)
$$

Note that this is not an infinitesimal variation and that it has exactly the form, consistent with Miwa parametrization used in the previous section 3 .

Since any bilinear combination can be generated in this way from $U(z)$, it is clear that the entire Hamiltonian $\sum A_{m n} \tilde{\psi}_{m} \psi_{n}$ can be also considered as resulting from some transformation of $V$ (i.e. of "time-variables" $t_{k}$ ). In other words,

$$
\tau\{A\}=\mathcal{O}_{A}[t] \tau\{A=U\}
$$

These operators $\mathcal{O}_{A}$ are naturally interpreted as elements of the group $G L(\infty)$, acting on the "Universal Grassmannian" [82], [83], 84], parametrized by the matrices $A_{m n}$ modulo changes of coordinates $z \rightarrow f(z)$. This representation for $\tau\{A\}$ is, however, not very convenient, and usually one considers infinitesimal version of the transformation, which just shifts $A$

$$
\tau\{t \mid A+\delta A\}=\hat{\mathcal{O}}_{\delta A}[t] \tau\{t \mid A\}
$$

note that this transformation clearly distinguishes between the dependencies of $\tau$ on $t$ and on all other components of $A$. The possibility of such representation with the privileged role of Cartan generators is the origin of all simplifications, arising in the case of free-fermion $\tau$-functions. ${ }^{29}$

\footnotetext{
${ }^{29}$ It is also the reason, why these are free-fermion tau-functions, that appear in the study of ordinary integrable hierarchies: the Hamiltonian flows, which describe evolution in different $t$-directions just commute, because $t$ 's are associated with the commuting Cartan generators of $G L(\infty)$. In the more general situation the flows would form closed but non - abelian algebra.
} 
Relation (4.15) is the basis of the orbit interpretation of $\tau$-functions [83]. It is also important to understand the role of the "string equation" and othert constraints, imposed on $\tau$-functions in the theory of matrix models. These arise as some particular subalgebras in the set of $\hat{\mathcal{O}}$-operators, and their role is to specify particular points $A$ in the Grassmannian, of which this subalgebra is a stabilizer. Po $^{0}$ The simplest examples are in fact provided by formulas from the section 2.3 above, where combinations of the screening charges describe $A$ 's, which are stable points of discrete Virasoro- and $W$-constraints (in the latter case the multi-fermion system is used).

The fact that the $\tau$-function at all the points $A$ of Grassmannian can be obtained by the group action from $\tau\{0\}$, has an implication, known as Hirota equation. The idea [83] is just that there are Casimir operators in the group, which commute with the group action and thus the eigenvalue of the Casimir operator is the same for $\tau\{A\}$ at all $A$. In the free-fermion case the simplest example of Casimir operator is given by

$$
J_{0}=\oint J=\oint \psi \tilde{\psi}=\sum_{n \in Z} \psi_{n} \tilde{\psi}_{n} .
$$

The eigenvalue of this operator for the vacuum state $|0\rangle$ is an infinite subtraction constant, and this makes equation $J_{0} \mathcal{O}_{A}|0\rangle=\mathcal{O}_{A} J_{0}|0\rangle=$ const $\cdot \mathcal{O}_{A} \mid$ $0\rangle$, or $J_{0} \tau\{A\}=$ const $\cdot \tau\{A\}$ not very interesting. However, this operator is represented in bilinear form and in such cases the following trick is usually usefull.

30 This relation is straightforward in the case of Virasoro constraints, since Virasoro algebra is just a subalgebra of the $G L(\infty)$ acting on $\tau$-functions, and thus is a symmetry (covariance) of associated integrable hierarchies [84]. $W$-constraints do not form Liesubalgebra of this $G L(\infty)$, they arise after certain reduction, which in turn exists in a simple form not everywhere on the Grassmannian (in particular $W$ is not a symmetry of entire KP hierarchy [85]): here we deal with a more sophisticated self-consistency relation, which remains to be understood in full details (e.g., it is unknown, whether reduction exists at least at any Virasoro-stable point, which would significantly simplify this kind of consideration). In fact, the entire relation between the constraints and $\tau$-functions is not exhaustively worked out: for example, there is still no clear and satisfactory proof, that the full set of Virasoro and/or $W$-constraints implies that partition function is a $\tau$-function, which would be pure algebraic and not refer to the uniqueness of solutions to the constraints. Result, widely discussed in the literature, see [29], is that string equation (the lowest Virasoro constraint $L_{-1} Z=0$ ), if imposed on $Z$, which is somehow known to be the properly reduced $\tau$-function, implies the entire set of Virasoro and $W$-constraints (though even this proof can still have some loopholes). 
If operator, which is bilinear in generators of the algebra, $T^{a} T^{a}$, commutes with the action of the group, so does $T^{a} \otimes T^{a}$, if the group action on tensor product of representations is defined as |\rangle$\otimes|\rangle \rightarrow \mathcal{O}_{A}|\rangle \otimes \mathcal{O}_{A}|\rangle$. (Indeed, then $\left(T^{a} \otimes I+I \otimes T^{a}\right)^{2}$ commutes with the group action and so does $T^{a} \otimes$ $T^{a}=\frac{1}{2}\left(\left(T^{a} \otimes I+I \otimes T^{a}\right)^{2}-T^{a} T^{a} \otimes I-I \otimes T^{a} T^{a}\right)$.) If further, $T^{a} \otimes T^{a}$ annihilates the product of two vacuum states:

$$
\left(T^{a} \otimes T^{a}\right)|0\rangle \otimes|0\rangle=0
$$

then the same equation holds for all $A$ :

$$
\left(T^{a} \otimes T^{a}\right)\left|\mathcal{O}_{A}\right\rangle \otimes\left|\mathcal{O}_{A}\right\rangle=0
$$

Condition (4.17) is trivially valid in our case:

$$
\sum_{n \in Z} \psi_{n}|0\rangle \otimes \tilde{\psi}_{n}|0\rangle=0
$$

since in every term in the sum one of the vacuum states is annihilated: the first one of $n \geq 0$ and the second one if $n<0$. Pr Thus we obtain the relation

$$
\sum_{n \in Z} \psi_{n}\left|\mathcal{O}_{A}\right\rangle \otimes \tilde{\psi}_{n}\left|\mathcal{O}_{A}\right\rangle=0
$$

which can be now multiplied from the left by

$$
\langle 0| \psi(\infty) e^{H_{\text {Cartan }}(t)} \otimes\langle 0| \tilde{\psi}(\infty) e^{H_{\text {Cartan }}\left(t^{\prime}\right)}
$$

$\left(t_{k}^{\prime}\right.$ do not need to coincide with $\left.t_{k}\right)$ and after insertions of $\psi$-operators are expressed as the shifts of times, we obtain:

$$
\sum_{n \in Z} D_{n}^{-} \tau\{t \mid A\} \otimes D_{n}^{+} \tau\{t \mid A\}=0,
$$

\footnotetext{
${ }^{31}$ It is easy to verify directly that $\sum_{n} \psi_{n} \otimes \tilde{\psi}_{n}$ is indeed a Casimir operator in the tensor product:

$$
\begin{aligned}
& {\left[\sum_{n} \psi_{n} \otimes \tilde{\psi}_{n}, I \otimes \sum_{l, m} A_{l m} \psi_{l} \tilde{\psi}_{m}+\sum_{l, m} A_{l m} \psi_{l} \tilde{\psi}_{m} \otimes I\right]=} \\
& =\sum_{n}\left(\psi_{n} \otimes \sum_{m} A_{n m} \tilde{\psi}_{m}-\sum_{l} A_{l n} \psi_{l} \otimes \tilde{\psi}_{n}\right)=\sum_{l} \sum_{m} A_{l m}\left(\psi_{l} \otimes \tilde{\psi}_{m}-\psi_{l} \otimes \tilde{\psi}_{m}\right)=0 .
\end{aligned}
$$
}


where $\sum_{n \geq 0} D_{n}^{ \pm} z^{-n}=\exp \left( \pm \sum_{k>0} \frac{1}{k z^{k}} \frac{\partial}{\partial t_{k}}\right)$. This is particular form of Hirota equation [86], which is often used to define $\tau$-functions, associated with integrable hierarchies. If one takes (4.8) for the definition, as it is more natural to do in the general "theory of everything" and as we did above, eq.(4.21) is the starting point for the path, leading to hierarchies in conventional form of differential equations, the Lax and pseudodifferential representations naturally appearing on the way. We do not go along this path in these notes.

The last remark to be made, before we proceed to more detailed formulas, is that $\tau$-functions can be considered as determinants Det $\bar{\partial}$ of the $\bar{\partial}$ operators acting on fields with some complicated boundary conditions (like $\psi(z) \sim \exp \left(\sum_{k>0} t_{k} z^{-k}\right)$ in the simplest case of $t$-dependencies $)$. Entire Adependence is usually described in this context as that on the point of the "universal module space", which once appeared in the study of string models on Riemann surface of arbitrary genus [87]. From this point of view more general $\tau$-functions are sections of the bundles over universal module space, associated with conformal models, more sophisticated then just the theory of free fermions (and b,c-systems). The WZNW model is, of course, the most important example to be studied in this context.

The crucial feature of all the quantites, associated in this way to conformal models is applicability of Wick theorem, reducing multipoint correlation functions to pair correlators. In the free fermion case this is just a consequence of quadratic form of Lagrangian, in generic situation this follows from existence of holomorphic operator algebra, which allows to define the correlators by fixation of monodromy properties, dictated by pairwise collision of points. Wick theorem is the concrete source of determinant formulas for tau-functions, which are used in order to establish their relations with matrix models and other branches of string theory.

After this discussion of the context where free-fermion $\tau$-functions can and do appear, we turn now to more detailed and exact formulas, relevant in this particular free-fermion case. They are mostly due to Japanese school [88], though many other people contributed to this field after it was established. The only sophisticated part of the work with these formulas is accurate accounting for the normal ordering routine which will be mostly unnecessary for our purposes. We shall mostly follow the presentation of papers [89], 30], [36]. 


\subsection{Basic determinant formula for the free-fermion cor- relator}

Let us consider the following matrix element:

$$
\tau_{N}\{t, \bar{t} \mid G\}=\left\langle N\left|e^{H} G e^{\bar{H}}\right| N\right\rangle
$$

where

$$
\begin{gathered}
\psi(z)=\sum_{n \in Z} \psi_{n} z^{n} d z^{1 / 2} ; \quad \tilde{\psi}(z)=\sum_{n \in Z} \tilde{\psi}_{n} z^{-n-1} d z^{1 / 2} ; \\
G=\exp \left(\sum_{m, n \in Z} A_{m n} \psi_{m} \tilde{\psi}_{n}\right) ; \\
H=\sum_{k>0} t_{k} J_{k}, \quad \bar{H}=\sum_{k>0} \bar{t}_{k} J_{-k} \\
J(z)=\psi(z) \tilde{\psi}(z)=\sum_{n \in Z} J_{n} z^{-n-1} d z ; \quad J_{n}=\sum_{k} \psi_{k} \tilde{\psi}_{k+n} ; \\
{\left[\tilde{\psi}_{m}, \psi_{n}\right]_{+}=\delta_{m, n} ; \quad\left[J_{m}, J_{n}\right]=m \delta_{m+n, 0} ;} \\
\psi_{m}|N\rangle=0, \quad m<N ; \quad\langle N| \psi_{m}=0, \quad m \geq N ; \\
\tilde{\psi}_{m}|N\rangle=0, \quad m \geq N ; \quad\langle N| \tilde{\psi}_{m}=0, \quad m<N ; \\
J_{m}|N\rangle=0, \quad m>0 ; \quad\langle N| J_{m}=0, \quad m<0 .
\end{gathered}
$$

The " $N$-th vacuum" $|N\rangle$ is defined as the Dirac sea, filled up to the level $N$ :

$$
\begin{array}{r}
|N\rangle=\prod_{i=N}^{\infty} \tilde{\psi}_{i}|\infty\rangle=\prod_{i=-\infty}^{N-1} \psi_{i}|-\infty\rangle \\
\langle N|=\langle\infty| \prod_{i=N}^{\infty} \psi_{i}=\langle-\infty| \prod_{i=-\infty}^{N-1} \tilde{\psi}_{i}
\end{array}
$$

where the "empty" (bare) and "completely filled" vacua are defined so that:

$$
\begin{aligned}
& \tilde{\psi}_{m}|-\infty\rangle=0, \quad\langle-\infty| \psi_{m}=0 \\
& \psi_{m}|\infty\rangle=0, \quad\langle\infty| \tilde{\psi}_{m}=0
\end{aligned}
$$

for any $m \in Z$. For the only reason that operators $J, H, \bar{H}$ and $G$ are defined so that they have usually $\tilde{\psi}$ at the very right and $\psi$ at the very left, 
we have also:

$$
\begin{aligned}
& J_{m}|-\infty\rangle=0, \quad\langle-\infty| J_{m}=0 \\
& G^{ \pm 1}|-\infty\rangle=|-\infty\rangle ; \quad\langle-\infty| G^{ \pm 1}=\langle-\infty| ; \\
& e^{ \pm \bar{H}}|-\infty\rangle=|-\infty\rangle ; \quad\langle-\infty| e^{ \pm H}=\langle-\infty| .
\end{aligned}
$$

Now we can use all these formulas to rewrite our original correlator (4.22) as:

$$
\begin{gathered}
\left\langle N\left|e^{H} G e^{\bar{H}}\right| N\right\rangle= \\
=\left\langle-\infty\left|\left(\prod_{i=-\infty}^{N-1} \tilde{\psi}_{i}\right) e^{H} G e^{\bar{H}}\left(\prod_{i=-\infty}^{N-1} \psi_{i}\right)\right|-\infty\right\rangle= \\
=\left\langle-\infty\left|e^{-H}\left(\prod_{i=-\infty}^{N-1} \tilde{\psi}_{i}\right) e^{H} G e^{\bar{H}}\left(\prod_{i=-\infty}^{N-1} \psi_{i}\right) e^{-\bar{H}}\right|-\infty\right\rangle= \\
=\left\langle-\infty\left|\prod_{i=-\infty}^{N-1} \tilde{\Psi}_{i}[t] \prod_{j=-\infty}^{N-1} \Psi_{j}^{G}[\bar{t}]\right|-\infty\right\rangle= \\
=\operatorname{Det}_{-\infty<i, j<N}\left\langle-\infty\left|\tilde{\Psi}_{i}[t] \Psi_{j}^{G}[\bar{t}]\right|-\infty\right\rangle= \\
=\operatorname{Det}_{i, j<0} \mathcal{H}_{i+N, j+N} .
\end{gathered}
$$

The last two steps here were introduction of " $G L(\infty)$-rotated" fermions,

$$
\tilde{\Psi}_{i}[t] \equiv e^{-H} \psi_{i} e^{H} ; \quad \Psi_{j}[\bar{t}] \equiv e^{\bar{H}} \psi_{j} e^{-\bar{H}} ; \quad \Psi_{j}^{G}[\bar{t}] \equiv G \Psi_{j}[\bar{t}] G^{-1}
$$

and application of the Wick theorem to express multifermion correlation function through pair correlators

$$
\begin{gathered}
\mathcal{H}_{i j}(t, \bar{t}) \equiv\left\langle-\infty\left|\tilde{\Psi}_{i}[t] \Psi_{j}^{G}[\bar{t}]\right|-\infty\right\rangle= \\
=\left\langle-\infty\left|\tilde{\Psi}_{i}[t] G \Psi_{j}[\bar{t}]\right|-\infty\right\rangle,
\end{gathered}
$$

(once again the fact that $G^{-1}|-\infty\rangle=|-\infty\rangle$ was used). The only non-trivial dynamical information entered through applicability of the Wick theorem, and for that it was crucial that all the operators $e^{H}, e^{\bar{H}}, G$ are quadratic exponents, i.e. can only modify the shape of the propagator, but do not destroy the quadratic form of the action (fields remain free). This is exactly equivalent to the statement that "heisenberg" operators $\Psi[t]$ are just "rotations" of $\psi$, i.e. that transformations (4.28) are linear. 
We shall now describe these transformations in a little more explicit form. Namely, their entire time-dependence can be encoded in terms of "Shur polinomials" $P_{n}(t)$. These are defined to have a very simple generating function (which we already encountered many times in the theory of matrix models):

$$
\sum_{n \geq 0} P_{n}(t) z^{n}=\exp \left(\sum_{k=1}^{\infty} t_{k} z^{k}\right)
$$

(i.e. $P_{0}=1, \quad P_{1}=t_{1}, \quad P_{2}=\frac{t_{1}^{2}}{2}+t^{2}$ etc.), and satisfy the relation

$$
\frac{\partial P_{n}}{\partial t_{k}}=P_{n-k}
$$

Since

$$
\exp \left(\sum_{k=1}^{\infty} t_{k} z^{k}\right)=\prod_{k>0}\left(\sum_{n_{k} \geq 0} \frac{1}{n_{k} !} t_{k}^{n_{k}} z^{k n_{k}}\right)
$$

Shur polinomials can be also represented as

$$
P_{n}(t)=\sum_{\substack{\left\{n_{k}\right\} \\ \sum_{k>0} k n_{k}=n}}\left(\prod_{k>0} \frac{1}{n_{k} !} t_{k}^{n_{k}}\right) .
$$

Now, since

$$
e^{-B} A e^{B}=A+[A, B]+\frac{1}{2 !}[[A, B], B]+\frac{1}{3 !}[[[A, B], B], B]+\ldots
$$

and

$$
\left[\tilde{\psi}_{i}, J_{k}\right]=\tilde{\psi}_{i+k}, \quad\left[\left[\tilde{\psi}_{i}, J_{k_{1}}\right], J_{k_{2}}\right]=\tilde{\psi}_{i+k_{1}+k_{2}}, \ldots,
$$

we have for every fixed $k$ :

$$
e^{-t_{k} J_{k}} \tilde{\psi}_{i} e^{t_{k} J_{k}}=\sum_{n_{k} \geq 0} \frac{t_{k}^{n_{k}}}{n_{k} !} \tilde{\psi}_{i+k n_{k}} .
$$


It remains to note that all the harmonics of $J$ in $H=\sum_{k>0} t_{k} J_{k}$ commute with each other, to obtain:

$$
\begin{gathered}
\tilde{\Psi}_{i}(t)=e^{-H} \tilde{\psi}_{i} e^{H}=\left(\prod_{k>0} e^{-t_{k} J_{k}}\right) \tilde{\psi}_{i}\left(\prod_{k>0} e^{t_{k} J_{k}}\right)= \\
=\sum_{n \geq 0} \tilde{\psi}_{i+n}\left(\sum_{\substack{\left\{n_{k}\right\} \\
\sum_{k>0} k n_{k}=n}}\left(\prod_{k>0} \frac{1}{n_{k} !} t_{k}^{n_{k}}\right)\right) \stackrel{(4.32)}{=} \\
=\sum_{n \geq 0} \tilde{\psi}_{i+n} P_{n}(t)=\sum_{l \geq i} \tilde{\psi}_{l} P_{l-i}(t) .
\end{gathered}
$$

Similarly, relation $\left[J_{k}, \psi_{j}\right]=\psi_{k+j}$ implies, that

$$
\Psi_{j}(\bar{t})=e^{\bar{H}} \psi_{j} e^{-\bar{H}}=\sum_{n \geq 0} \psi_{j+n} P_{n}(\bar{t})=\sum_{m \geq j} \psi_{m} P_{m-j}(\bar{t})
$$

and finally"z

$$
\begin{aligned}
& \mathcal{H}_{i j}=\sum_{\substack{l \geq i \\
m \geq j}}\left\langle-\infty\left|\tilde{\psi}_{l} G \psi_{m}\right|-\infty\right\rangle P_{l-i}(t) P_{m-j}(\bar{t})= \\
& =\sum_{\substack{l \geq i \\
m \geq j}} T_{l m} P_{l-i}(t) P_{m-j}(\bar{t}),
\end{aligned}
$$

which implies also that

$$
\begin{aligned}
\frac{\partial \mathcal{H}_{i j}}{\partial t_{k}} & =\mathcal{H}_{i+k, j} \\
\frac{\partial \mathcal{H}_{i j}}{\partial \bar{t}_{k}} & =\mathcal{H}_{i, j+k}
\end{aligned}
$$

Matrix

$$
T_{l m} \equiv\left\langle-\infty\left|\tilde{\psi}_{l} G \psi_{m}\right|-\infty\right\rangle
$$

${ }^{32}$ Eqs. (4.34) can be also interpreted as representations of Shur polinomials in terms of fermionic correlators in the bare vacuum:

$$
\begin{aligned}
& P_{m}(\bar{t})=\left\langle-\infty\left|\tilde{\psi}_{j+m} e^{\bar{H}} \psi_{j}\right|-\infty\right\rangle \\
& P_{m}(t)=\left\langle-\infty\left|\tilde{\psi}_{i} e^{H} \psi_{i+m}\right|-\infty\right\rangle
\end{aligned}
$$


is the one which defines fermion rotations under the action of $G L(\infty)$-group element $G$ :

$$
\begin{aligned}
& G \psi_{m} G^{-1}=\sum_{l \in Z} \psi_{l} T_{l m} ; \\
& G^{-1} \tilde{\psi}_{l} G=\sum_{m \in Z} T_{l m} \tilde{\psi}_{m}, \text { or } G \tilde{\psi}_{l} G^{-1}=\sum_{m \in Z}\left(T^{-1}\right)_{l m} \tilde{\psi}_{m} .
\end{aligned}
$$

If $G=1, T_{l m}=\delta_{l m}$. If all $t_{k}=\bar{t}_{k}=0, \mathcal{H}_{i j}=T_{i j}$.

\subsection{Toda-lattice $\tau$-function and linear reductions of Toda hierarchy}

In the previous subsection we derived a formula

$$
\tau_{N}\{t, \bar{t} \mid G\}=\operatorname{Det}_{i, j<0} \mathcal{H}_{i+N, j+N}
$$

for the basic correlator, which defines "Toda-lattice $\tau$-function". For obvious reasons $\bar{t}$ are often refered to as negative-times. $\tau$-function can be normalized by dividing over the same quantity for all time-variables vanishing, but this is not always convenient. Eq. (4.39) has generalizations - when similar matrix elements in a multifermion system is considered - this leads to "multicomponent Toda" (or AKNS) $\tau$-functions. Generalizations to arbitrary conformal models should be considered as well. It has also particular "reductions", of which the most important are: KP (Kadomtsev-Petviashvili), forced (semiinfinite) and Toda-chain $\tau$-functions. This is the subject to be discussed in this subsection.

Idea of linear reduction is that the form of operator $G$, or, what is the same, of the matrix $T_{l m}$ in eq.(4.35), can be adjusted in such a way, that $\tau_{N}\{t, \bar{t} \mid G\}$ becomes independent of some variables, i.e. equation(s)

$$
\left(\sum_{k} \alpha \frac{\partial}{\partial t_{k}}+\sum_{k} \bar{\alpha} \frac{\partial}{\partial \bar{t}_{k}}+\sum_{k} \beta_{k} D_{N}(k)+\gamma\right) \tau_{N}\{t, \bar{t} \mid G\}=0
$$

can be solved as equations for $G$ for all the values of $t, \bar{t}$ and $N$ at once. (In (4.40) $D_{N}(k) f_{N} \equiv f_{N+k}-f_{N}$.) In this case the system of integrable equations (hierarchy), arising from Hirota equation for $\tau$, gets reduced and one usually speaks about "reduced hierarchy". Usually equation (4.40) is imposed directly on matrix $\mathcal{H}_{i j}$, of course than (4.40) is just a corollary. 
We shall refer to the situation when (4.40) is fulfilled for any $t, \bar{t}, N$ as to "strong reduction". It is often reasonable to consider also "weak reductions", when (4.40) is satisfied on particular infinite-dimensional hyperplanes in the space of time-variables. Weak reduction is usually a property of entire $\tau$-function as well, but not expressible in the from of a local linear equation, satisfied identicall for all values of $t, \bar{t}, N$. Now we proceed to concrete examples:

Toda-chain hierarchy. This is a strong reduction. The corresponding constraint (4.40) is just

$$
\frac{\partial \mathcal{H}_{i j}}{\partial t_{k}}=\frac{\partial \mathcal{H}_{i j}}{\partial \bar{t}_{k}}
$$

or, because of (4.36), $\mathcal{H}_{i+k, j}=\mathcal{H}_{i, j+k}$. It has an obvious solution:

$$
\mathcal{H}_{i, j}=\hat{\mathcal{H}}_{i+j},
$$

i.e. $\mathcal{H}_{i j}$ is expressed in terms of a one-index quantity $\hat{\mathcal{H}}_{i}$. It is, however, not enough to say, what are restrictions on $\mathcal{H}_{i j}$ - they should be fulfiled for all $t$ and $\bar{t}$ at once, i.e. should be resolvable as equations for $T_{l m}$. In the case under consideration this is simple: $T_{l m}$ should be such that

$$
T_{l m}=\hat{T}_{l+m} .
$$

Indeed, then

$$
\begin{aligned}
& \mathcal{H}_{i j}=\sum_{l, m} T_{l m} P_{l-i}(t) P_{m-j}(\bar{t})=\sum_{l, m} \hat{T}_{l+m} P_{l-i}(t) P_{m-j}(\bar{t})= \\
& =\sum_{n \geq 0} \hat{T}_{n+i+j}\left(\sum_{k=0}^{n} P_{k}(t) P_{n-k}(\bar{t})\right),
\end{aligned}
$$

and

$$
\hat{\mathcal{H}}_{i}=\sum_{n \geq 0} \hat{T}_{n+i}\left(\sum_{k=0}^{n} P_{k}(t) P_{n-k}(\bar{t})\right) .
$$

Volterra hierarchy. Toda-chain $\tau$-function can be further weakly reduced to satisfy the identity

$$
\left.\frac{\partial \tau_{2 N}}{\partial t_{2 k+1}}\right|_{\left\{t_{2 l+1}=0\right\}}=0, \quad \text { for all } k,
$$


i.e. $\tau_{2 N}$ is requested to be even function of all odd-times $t_{2 l+1}$ (this is an example of "global characterization" of the weak reduction). Note that (4.45) is imposed only on Toda-chain $\tau$-function with even values of zero-time. Then (4.45) will hold whenever $\hat{\mathcal{H}}_{i}$ in (4.44) are even (odd) functions of $t_{\text {odd }}$ for even (odd) values of $i$. Since Shur polinomials $P_{k}(t)$ are even (odd) functions of odd-times for even (odd) $k$, it is enough that the sum in (4.44) goes over even (odd) $n$ when $i$ is even (odd). In other words, the restriction on $T_{l m}$ is that

$$
T_{l m}=\hat{T}_{l+m}, \quad \text { and } \quad \hat{T}_{2 k+1}=0 \text { for all } k .
$$

Forced hierarchies. This is another important example of strong reduction. It also provides an example of singular $\tau$-functions, arising when $G=\exp \left(\sum A_{m n} \psi_{m} \tilde{\psi}_{n}\right)$ blows up and normal ordered operators should be used to define regularized $\tau$-functions. Forced hierarchy appears when $G$ can be represented in the form [89] $G=G_{0} P_{+}$, where projection operator $P_{+}$is such that

$$
\begin{array}{r}
P_{+}|N\rangle=|N\rangle \text { for } N \geq N_{0}, \\
P_{+}|N\rangle=0 \text { for } N<N_{0} .
\end{array}
$$

Explicit expression for this operator is ${ }^{33}$

$$
P_{+}=: \exp \left(-\sum_{l<N_{0}} \tilde{\psi}_{l} \psi_{l}\right):=\prod_{l<N_{0}}\left(1-\tilde{\psi}_{l} \psi_{l}\right)=\prod_{l<N_{0}} \psi_{l} \tilde{\psi}_{l}
$$

Because of (4.47), $\left.P_{+}|-\infty\rangle=\right)$, and the identity $G|-\infty\rangle=|-\infty\rangle$, which was essentially used in the derivation in (4.27), can be satisfied only if $G_{0}$ is singular and $T_{l m}=\infty$. In order to avoid this problem one usually introduces in the vicinity of such singular points in the universal module space a sort of normalized (forced) $\tau$-function $\tau_{N}^{f} \equiv \frac{\tau_{N}}{\tau_{N_{0}}}$. One can check that now $T_{l m}^{f}=\infty$ for all $l, m<N_{0}$, and $\tau^{f}$ can be represented as determinant of a finaldimensional matrix [90], [89]:

$$
\tau_{N}^{f}=\operatorname{Det}_{N_{0} \leq i, j<N} \mathcal{H}_{i j}^{f} \quad \text { for } N>N_{0}
$$

\footnotetext{
${ }^{33}$ Normal ordering sign : : means that all operators $\tilde{\psi}$ stand to the left of all operators $\psi$. The product at the r.h.s. obviously implies both the property (4.47) and projection property $P_{+}^{2}=P_{+}$.
} 


$$
\begin{aligned}
& \tau_{N_{0}}^{f}=1 ; \\
& \tau_{N}^{f}=0 \text { for } N<N_{0} .
\end{aligned}
$$

For $N>N_{0}$ we have now determinant of a finite-dimensional $\left(N-N_{0}\right) \times$ $\left(N-N_{0}\right)$ matrix. The choice of $N_{0}$ is not really essential, therefore it is better to put $N_{0}=0$ in order to simplify formulas, phraising and relation with the discrete matrix models $\left(N_{0}\right.$ is easily restored if everywhere $N$ is substituted by $\left.N-N_{0}\right)$. For forced hierarchies one can also represent $\hat{\tau}$ as

$$
\tau_{N}^{f}=\operatorname{Det}_{0 \leq i, j<N} \partial_{1}^{i} \bar{\partial}_{1}^{j} \mathcal{H}^{f}
$$

where $\mathcal{H}^{f}=\mathcal{H}_{00}^{f}$ and $\partial_{1}=\frac{\partial}{\partial t_{1}}, \bar{\partial}_{1}=\frac{\partial}{\partial \bar{t}_{1}}$. For forced Toda-chain hierarchy this turns into even simpler expression:

$$
\tau_{N}^{f}=\operatorname{Det}_{0 \leq i, j<N} \partial_{1}^{i+j} \hat{\mathcal{H}}^{f},
$$

while for the forced Volterra case we get a product of two Toda-chain $\tau$ functions with twice as small value of $N$ 91]:

$$
\begin{aligned}
\tau_{2 N}^{f} & =\left(\operatorname{Det}_{0 \leq i, j<N} \partial_{2}^{i+j} \hat{\mathcal{H}}^{f}\right) \cdot\left(\operatorname{Det}_{0 \leq i, j<N} \partial_{2}^{i+j}\left(\partial_{2} \hat{\mathcal{H}}^{f}\right)\right)= \\
& =\tau_{N}^{f}\left[\hat{\mathcal{H}}^{f}\right] \cdot \tau_{N}^{f}\left[\partial_{2} \hat{\mathcal{H}}^{f}\right] .
\end{aligned}
$$

Forced $\tau_{N}^{f}$ can be always represented in the form of a scalar-product matrix model. Indeed,

$$
\mathcal{H}_{i j}=\sum T_{l m} P_{l-i}(t) P_{m-j}(\bar{t})=\oint \oint e^{U(h)+\bar{U}(\bar{h})} h^{i} \bar{h}^{j} T(h, \bar{h}) d h d \bar{h},
$$

where $T(h, \bar{h}) \equiv \sum_{l m} T_{l m} h^{-l-1} \bar{h}^{-m-1}$, and $e^{U(h)}=e^{\sum_{k>0} t_{k} h^{k}}=\sum_{l \geq 0} h^{l} P_{l}(t)$. Then, since $\operatorname{Det}_{0 \leq i, j<N} h^{i}=\Delta_{N}(h)$ - this is where it is essential that the hierarchy is forced -

$$
\operatorname{Det}_{0 \leq i, j<N} \mathcal{H}_{i j}=\prod_{i} \oint \oint e^{U\left(h_{i}\right)+\bar{U}\left(\bar{h}_{i}\right)} T\left(h_{i}, \bar{h}_{i}\right) d h_{i} d \bar{h}_{i} \cdot \Delta_{N}(h) \Delta_{N}(\bar{h}),
$$

i.e. we obtain a scalar-product model with

$$
d \mu_{h, \bar{h}}=e^{U(h)+\bar{U}(\bar{h})} T(h, \bar{h}) d h d \bar{h} .
$$


Inverse is also true: partition function of every scalar-product model is forced Toda-lattice $\tau$-function - see section 4.7 for more details.

$K P$ hierarchy. In this case we just ignore the dependence of $\tau$-function on times $\bar{t}$. Every Toda-lattice $\tau$-function can be considered also as KP $\tau$-function: just operator $G^{K P} \equiv G e^{\bar{H}}$ (a point of Grassmannian) becomes $\bar{t}$ dependent. Usually $N$-dependence is also eliminated - this can be considered as a little more sophisticated change of $G$. When $N$ is fixed, extra changes of field-variables are allowed, including transformation from Ramond to NeveuSchwarz sector etc. Often KP hierarchy is from the very beginning formulated in terms of Neveu-Schwarz (antiperiodic) fermionic fields (associated with principal representations of Kac-Moody algebras), i.e. expansions in the first line of (4.23) are in semi-integer powers of $z: \psi_{N S}(z)=\sum_{n \in Z} \psi_{n} z^{n-\frac{1}{2}} d z^{1 / 2}$.

Given a KP $\tau$-function one can usually construct a Toda-lattice one with the same $\mathrm{G}$, by introducing in appropriate way dependencies on $\bar{t}$ and $N$. For this purpose $\tau^{K P}$ should be represented in the form of (4.39):

$$
\tau^{K P}\{t \mid G\}=\operatorname{Det}_{i, j<0} \mathcal{H}_{i j}^{K P},
$$

where $\mathcal{H}_{i j}^{K P}=\sum_{l} T_{l j} P_{l-i}(t)$. Since $T_{l m}$ is a function of $G$ only, it does not change when we built up a Toda-lattice $\tau$-function:

$$
\begin{aligned}
& \tau_{N}\{t, \bar{t} \mid G\}=\operatorname{Det}_{i, j<0} \mathcal{H}_{i+N, j+N} \\
& \mathcal{H}_{i j}=\sum_{l, m} T_{l m} P_{l-i}(t) P_{m-j}(\bar{t})=\sum_{m} \mathcal{H}_{i m}^{K P} P_{m-j}(\bar{t}) .
\end{aligned}
$$

Then

$$
\tau^{K P}\{t \mid G\}=\tau_{0}\{t, 0 \mid G\} .
$$

If we go in the opposite direction, when Toda-lattice $\tau$-function is considered as KP $\tau$-function,

$$
\begin{array}{r}
\tau_{0}\{t, \bar{t} \mid G\}=\tau^{K P}\{t \mid \tilde{G}(\bar{t})\} ; \\
\tilde{\mathcal{H}}_{i j}^{K P}=\sum_{m} \mathcal{H}_{i m} P_{m-j}(\bar{t}) \text { and } \\
\tilde{T}_{l j}\{\tilde{G}(\bar{t})\}=\sum_{m} T_{l m}\{G\} P_{m-j}(\bar{t}) .
\end{array}
$$

$\mathrm{KP}$ reduction in its turn has many further weak reductions ( $\mathrm{KdV}$ and Boussinesq being the simplest examples). We shall mention them again in section 4.9 below, after Miwa transformation of representation (4.39) will be considered in the next subsection. 


\subsection{Fermion correlator in Miwa coordinates}

Let us now return to original correlator (4.22) and discuss in a little more details the implications of bosonization identity (4.13). In order not to write down integrals of $J$, we introduce scalar field:阮

$$
\phi(z)=\sum_{\substack{k \neq 0 \\ k \in Z-0}} \frac{J_{-k}}{k} z^{k}+\phi_{0}+J_{0} \log z,
$$

such that $\partial \phi(z)=J(z)$. Then (4.13) states that:

$$
: \psi(\lambda) \tilde{\psi}(\tilde{\lambda}):=: e^{\phi(\tilde{\lambda})-\phi(\lambda)}:
$$

"Normal ordering" here means nothing more but the requirement to neglect all mutual contractions (or correlators) of operators in between : : when Wick theorem is applied to evaluate corrletion functions. One can also get rid of the normal ordering sign at the l.h.s. of (4.60), then

$$
\psi(\lambda) \tilde{\psi}(\tilde{\lambda})=: e^{\phi(\tilde{\lambda})}:: e^{-\phi(\lambda)}:
$$

In distinguished coordinates on a sphere, when the free field propagator is just $\log (z-\tilde{z})$, one also has:

$$
\psi(z) \tilde{\psi}(\tilde{z})=\frac{1}{z-\tilde{z}}: \psi(z) \tilde{\psi}(\tilde{z}):
$$

Our task now is to express operators $e^{H}$ and $e^{\bar{H}}$ through the field $\phi$. This is simple:

$$
H=\oint_{0} U(z) J(z)=\oint_{0} U(z) \partial \phi(z)=-\oint_{0} \phi(z) \partial U(z) .
$$

Here as usual $U(z)=\sum_{k>0} t_{k} z^{k}$ and integral is around $z=0$. This is very similar to generic linear functional of $\phi_{-}(\lambda) \equiv-\sum_{k>0} \frac{1}{k} J_{k} \lambda^{-k}$,

$$
H=\int \phi_{-}(\lambda) f(\lambda) d \lambda
$$

\footnotetext{
34 One can consider $\phi$ as introduced for simplicity of notation, but it should be kept in mind that the scalar-field representation is in fact more fundamental for generic $\tau$ functions, not related to the level $k=1$ Kac-Moody algebras (this phenomenon is well known in conformal filed theory, see [16] for more details).
} 
one should only require that

$$
\partial U(z)=\int \frac{f(\lambda)}{z-\lambda} d \lambda
$$

i.e.

$$
U(z)=\int \log \left(1-\frac{z}{\lambda}\right) f(\lambda) d \lambda
$$

In terms of time-variables this means that

$$
t_{k}=-\frac{1}{k} \int \lambda^{-k} f(\lambda) d \lambda
$$

Here we required that $U(z=0)=0$, sometimes it can be more natural to introduce also

$$
t_{0}=\int \log \lambda f(\lambda) d \lambda
$$

This change from the time-variables to "time density" $f(\lambda)$ is known as Miwa transformation. In order to establish relation with fermionic representation and also with matrix models we shall need it in "discretized" form:

$$
\begin{aligned}
t_{k} & =\frac{\xi}{k}\left(\sum_{\gamma} \lambda_{\gamma}^{-k}-\sum_{\gamma} \tilde{\lambda}_{\gamma}^{-k}\right), \\
t_{0} & =-\xi\left(\sum_{\gamma} \log \lambda_{\gamma}-\sum_{\gamma} \log \tilde{\lambda}_{\gamma}\right) .
\end{aligned}
$$

We changed integral over $\lambda$ for a discrete sum (i.e. the density function $f(\lambda)$ is a combination of $\delta$-functions, picked at some points $\lambda_{\gamma}, \tilde{\lambda}_{\gamma}$. This is of course just another basis in the space of the linear functionals, but the change from one basis to another one is highly non-trivial. The thing is, that we selected the basis where amplitudes of different $\delta$-functions are the same: parameter $\xi$ in (4.67) is independent of $\gamma$. Thus the real parameters are just positions of the points $\lambda_{\gamma}, \tilde{\lambda}_{\gamma}$, while the amplitude is defined by the density of these points in the integration (summation) domain. This domain does not need to be a priori specified: it can be real line, any other contour or - better - some Riemann surface.) Parameter $\xi$ is also unnecessary

\footnotetext{
${ }^{35}$ As it is usual nowadays, a factor of $2 \pi i$ is assumed to be included into the definition of contour integral $\oint$.
} 
to introduce, because basises with different $\xi$ are essentially equivalent. We shall soon put it equal to one, but not before Miwa transformation will be discussed in a little more detail.

Our next steps will be as follows. Substitution of (4.63) into (4.67), gives:

$$
H=-\xi \sum_{\gamma} \phi_{-}\left(\lambda_{\gamma}\right)+\xi \sum_{\gamma} \phi_{-}\left(\tilde{\lambda}_{\gamma}\right) .
$$

In fact, what we need is not operator $H$ itself, but the state which is created when $e^{H}$ acts on the vacuum state $\langle N|$. Then, since $\langle N| J_{m}=0$ for $m<0$, $\langle N| e^{-\xi \phi_{-}(\lambda)}$ is essentially equivalent to $\langle N| e^{-\xi \phi(\lambda)}$ with $\phi_{-}(\lambda)$ substituted by entire $\phi(\lambda)$. If $\xi=1, e^{-\phi(\lambda)}$ can be further changed for $\psi(\lambda)$ and we obtain an expression for the correlator (4.22) an expression where $e^{H}$ is substituted by a product of operators $\psi\left(\lambda_{\gamma}\right)$. The same is of course true for $e^{\bar{H}}$. Then Wick therem can be applied and a new type of determinant formulas arises like, for example,

$$
\tau \sim \frac{\Delta(\lambda, \tilde{\lambda})}{\Delta^{2}(\lambda) \Delta^{2}(\tilde{\lambda})} \operatorname{det}_{\gamma \delta}\left\langle N\left|\psi\left(\lambda_{\gamma}\right) \tilde{\psi}\left(\tilde{\lambda}_{\delta}\right) G\right| N\right\rangle
$$

It can be also obtained directly from (4.27), (4.29) and (4.35) by Miwa transformation. The rest of this subsection describes this derivation in somewhat more details.

The first task is to substitute $\phi_{-}$by $\phi$. For this purpose we introduce operator

$$
\sum_{k=-\infty}^{\infty} t_{k} J_{k}=H_{+}+H_{-}
$$

where $H_{+}=\sum_{k>0} t_{k} J_{k}$ is just our old $H, H_{-}=\sum_{k \geq 0} t_{-k} J_{k}$, and "negative times" $t_{-k}$ are defined by "analytical continuation" of the same formulas (4.65) and (4.67):

$$
t_{-k}=\frac{1}{k} \int \lambda^{k} f(\lambda) d \lambda=-\frac{\xi}{k}\left(\sum_{\gamma} \lambda_{\gamma}^{k}-\sum_{\gamma} \tilde{\lambda}_{\gamma}^{k}\right) .
$$

Then

$$
\sum_{k=-\infty}^{\infty} t_{k} J_{k}=H_{+}+H_{-}=-\xi\left(\sum_{\gamma} \phi\left(\lambda_{\gamma}\right)-\sum_{\gamma} \phi\left(\tilde{\lambda}_{\gamma}\right)\right)
$$


Further,

$$
e^{H_{+}+H_{-}}=e^{-\frac{1}{2} s(t)} e^{H_{+}} e^{H_{-}}=e^{\frac{1}{2} s(t)} e^{H_{-}} e^{H_{+}}
$$

where

$$
\begin{gathered}
s(t) \equiv \sum_{k>0} k t_{k} t_{-k}=-\xi^{2} \sum_{k>0} \frac{1}{k}\left(\sum_{\gamma}\left(\lambda_{\gamma}^{-k}-\tilde{\lambda}_{\gamma}^{-k}\right) \sum_{\delta}\left(\lambda_{\delta}^{k}-\tilde{\lambda}_{\delta}^{k}\right)\right)= \\
=\xi^{2} \log \left(\prod_{\gamma, \delta}^{\prime} \frac{\left(1-\frac{\lambda_{\delta}}{\lambda_{\gamma}}\right)\left(1-\frac{\tilde{\lambda}_{\delta}}{\tilde{\lambda}_{\gamma}}\right)}{\left(1-\frac{\tilde{\lambda}_{\delta}}{\lambda_{\gamma}}\right)\left(1-\frac{\lambda_{\delta}}{\tilde{\lambda}_{\gamma}}\right)}\right)+\text { const, }
\end{gathered}
$$

where prime means that the terms with $\gamma=\delta$ are excluded from the product in the numerator and accounted for in the infinite "constant", added at the r.h.s. In other words,

$$
\begin{aligned}
e^{\frac{1}{2} s(t)} & =\text { const } \cdot\left(\frac{\prod_{\gamma>\delta}\left(\lambda_{\gamma}-\lambda_{\delta}\right)\left(\tilde{\lambda}_{\gamma}-\tilde{\lambda}_{\delta}\right)}{\prod_{\gamma} \prod_{\delta}\left(\lambda_{\gamma}-\tilde{\lambda}_{\delta}\right)}\right)^{\xi^{2}}= \\
& =\text { const } \cdot\left(\frac{\Delta^{2}(\lambda) \Delta^{2}(\tilde{\lambda})}{\Delta(\lambda, \tilde{\lambda})}\right)^{\xi^{2}} \cdot
\end{aligned}
$$

Since $\langle N| J_{m}=0$ for all $m<0$, we have $\langle N| e^{H_{-}}=\langle N|$, and therefore

$$
\langle N| e^{H} \equiv\langle N| e^{H_{+}}=\langle N| e^{H_{-}} e^{H_{+}}=e^{-\frac{1}{2} s(t)}\langle N| e^{H_{+}+H_{-}} .
$$

From eq. (4.72),

$$
e^{H_{+}+H_{-}}=\mathrm{const} \cdot \prod_{\gamma}: e^{-\xi \phi\left(\lambda_{\gamma}\right)}:: e^{\xi \phi\left(\tilde{\lambda}_{\gamma}\right)}:
$$

where "const" is exactly the same as in (4.75). If $\xi=1$, eq.(4.61) can be used to write:

$$
\langle N| e^{H}=\frac{\Delta(\lambda, \tilde{\lambda})}{\Delta^{2}(\lambda) \Delta^{2}(\tilde{\lambda})}\langle N| \prod_{\gamma} \psi\left(\lambda_{\gamma}\right) \prod_{\gamma} \tilde{\psi}\left(\tilde{\lambda}_{\gamma}\right)
$$

36 The choice of $\xi$ can be dictated by particular purposes. Here we impose the requirement on Miwa transform to represent $e^{H}=e^{H_{\text {Cartan }}}$ as a product of dimension-1/2 operators - this is most natural from the point of view of Hirota equations and simplifies the relation with integrable hierarchies. However, in section 2.7 and 2.8 we used another requirement (and there $\xi=\frac{1}{\sqrt{2}}$ rather than $\xi=1$ ). There the 1 -matrix model was considered, which is characterized by especially simple form of the full hamiltonian (product of dimension-zero operators), and it was more important to adjust operators, which arise 
Similarly,

$$
e^{\bar{H}}|N\rangle=\prod_{\delta} \psi\left(\bar{\lambda}_{\delta}\right) \prod_{\delta} \tilde{\psi}\left(\tilde{\bar{\lambda}}_{\delta}\right)|N\rangle \frac{\Delta(\bar{\lambda}, \tilde{\bar{\lambda}})}{\Delta^{2}(\bar{\lambda}) \Delta^{2}(\tilde{\bar{\lambda}})}
$$

where

$$
\bar{t}_{k}=-\frac{1}{k} \sum_{\delta}\left(\bar{\lambda}_{\delta}^{k}-\tilde{\bar{\lambda}}_{\delta}^{k}\right)
$$

and we used the fact that $J_{m}|N\rangle=0$ for all $m>0$. Finaly,

$$
\begin{gathered}
\tau_{N}\{t, \bar{t} \mid G\}=\left\langle N\left|e^{H} G e^{\bar{H}}\right| N\right\rangle=\frac{\Delta(\lambda, \tilde{\lambda})}{\Delta^{2}(\lambda) \Delta^{2}(\tilde{\lambda})} \frac{\Delta(\bar{\lambda}, \tilde{\bar{\lambda}})}{\Delta^{2}(\bar{\lambda}) \Delta^{2}(\tilde{\bar{\lambda}})} \cdot \\
\cdot\left\langle N\left|\prod_{\gamma} \psi\left(\lambda_{\gamma}\right) \prod_{\gamma} \tilde{\psi}\left(\tilde{\lambda}_{\gamma}\right) G \prod_{\delta} \psi\left(\bar{\lambda}_{\delta}\right) \prod_{\delta} \tilde{\psi}\left(\tilde{\bar{\lambda}}_{\delta}\right)\right| N\right\rangle .
\end{gathered}
$$

Singularities at the coinciding points are completely eliminated from this expression, since poles and zeroes of the correlator are canceled by those coming from the Van-der-Monde determinants.

Let us now put $N=0$ and define normalized $\tau$-function

$$
\hat{\tau}_{0}\{t, \bar{t} \mid G\} \equiv \frac{\tau_{0}\{t, \bar{t} \mid G\}}{\tau_{0}\{0,0 \mid G\}},
$$

i.e. divide r.h.s. of (4.81) by $\langle 0|G| 0\rangle$. Wick theorem now allows to rewrite the correlator at the r.h.s. as a determinant of the block matrix:

$$
\operatorname{det}\left(\begin{array}{cc}
\frac{\left\langle 0\left|\psi\left(\lambda_{\gamma}\right) \tilde{\psi}\left(\tilde{\lambda}_{\delta}\right) G\right| 0\right\rangle}{\langle 0|G| 0\rangle} & \frac{\left\langle 0\left|\psi\left(\lambda_{\gamma}\right) G \tilde{\psi}^{(}\left(\tilde{\bar{\lambda}}_{\delta}\right)\right| 0\right\rangle}{\langle 0|G| 0\rangle} \\
-\frac{\left\langle 0\left|\tilde{\psi}\left(\tilde{\lambda}_{\delta}\right) G \psi\left(\bar{\lambda}_{\gamma}\right)\right| 0\right\rangle}{\langle 0|G| 0\rangle} & \frac{\left\langle 0\left|G \psi\left(\bar{\lambda}_{\gamma}\right) \tilde{\psi}\left(\tilde{\bar{\lambda}}_{\delta}\right)\right| 0\right\rangle}{\langle 0|G| 0\rangle}
\end{array}\right)
$$

from $e^{H_{\text {Cartan }}}$ after Miwa transform to have simple correlators with $e^{A \psi \tilde{\psi}}$. When analyzing the 1-matrix model from this point of view one should also keep in mind that it was actually represented in s.2.3 in terms of two complex fermions. Screening charges are $Q^{(+)}=\oint e^{\sqrt{2} \phi}=\oint \tilde{\psi}_{1} \psi_{2}=\oint e^{\phi_{1}-\phi_{2}}, \quad Q^{(-)}=\oint e^{-\sqrt{2} \phi}=\oint \tilde{\psi}_{2} \psi_{1}=\oint e^{\phi_{2}-\phi_{1}}$, while $\phi=\frac{1}{\sqrt{2}}\left(\phi_{1}-\phi_{2}\right)$. The Hamiltonian is $H_{\text {Cartan }}=\frac{1}{\sqrt{2}} \sum_{k} t_{k} J_{k}=\frac{1}{2} \sum_{k} t_{k}\left(J_{k}^{1}-J_{k}^{2}\right)$, and Miwa transformation generators insertions of operators $\chi_{1} \tilde{\chi}_{2}$, where $\chi_{1}$ and $\tilde{\chi}_{2}$ have dimension $1 / 8$ (rather than $1 / 2$ as in the one (complex)-fermion system, considered in this section). 
Special choices of points $\lambda_{\gamma}, \ldots, \tilde{\bar{\lambda}}_{\delta}$ can lead to simpler formulas. If $\tilde{\bar{\lambda}}_{\gamma} \rightarrow$ $\bar{\lambda}_{\gamma}$, so that $\bar{t}_{k} \rightarrow 0$, the matrix elements at the right lower block in (4.83) blow up, so that the off-diagonal blocks can be neglected. Then

$$
\begin{gathered}
\tau_{0}\{t, \bar{t} \mid G\} \rightarrow \tau^{K P}\{t \mid G\}=\frac{\left\langle 0\left|e^{H} G\right| 0\right\rangle}{\langle 0|G| 0\rangle}= \\
=\frac{\Delta(\lambda, \tilde{\lambda})}{\Delta^{2}(\lambda) \Delta^{2}(\tilde{\lambda})} \operatorname{det}_{\gamma \delta} \frac{\left\langle 0\left|\psi\left(\lambda_{\gamma}\right) \tilde{\psi}\left(\tilde{\lambda}_{\delta}\right) G\right| 0\right\rangle}{\langle 0|G| 0\rangle} .
\end{gathered}
$$

This function no longer depends on $\bar{t}$-times and is just a $\mathrm{KP} \tau$-function.

Matrix element

$$
\varphi(\lambda, \tilde{\lambda})=\frac{\langle 0|\psi(\lambda) \tilde{\psi}(\tilde{\lambda}) G| 0\rangle}{\langle 0|G| 0\rangle}
$$

is singular, when $\lambda \rightarrow \tilde{\lambda}: \varphi(\lambda, \tilde{\lambda}) \rightarrow \frac{1}{\lambda-\lambda}$. If now in (4.84) all $\tilde{\lambda} \rightarrow \infty$,

$$
\tau^{K P}\{t \mid G\}=\frac{\operatorname{det}_{\gamma \delta} \varphi_{\delta}\left(\lambda_{\gamma}\right)}{\Delta(\lambda)},
$$

where

$$
\varphi_{\delta}(\lambda) \equiv\left\langle 0\left|\psi(\lambda)\left(\partial^{\delta-1} \tilde{\psi}\right)(\infty) G\right| 0\right\rangle \sim \lambda^{\delta-1}\left(1+\mathcal{O}\left(\frac{1}{\lambda}\right)\right) .
$$

This is the main determinant representation of $\mathrm{KP} \tau$-function in Miwa parametrization.

Starting from representation (4.86) one can restore the corresponding matrix $\mathcal{H}_{i j}^{K P}$ in eq.(4.55) [36]:

$$
\mathcal{H}_{i j}^{K P}\{t\}=\oint z^{i} \varphi_{-j}(z) e^{\sum_{k} t_{k} z^{k}} d z,
$$

i.e.

$$
T_{l j}^{K P}=\oint z^{l} \varphi_{-j}(z)
$$

Then obviously $\frac{\partial \mathcal{H}_{i j}^{K P}}{\partial t_{k}}=\mathcal{H}_{i+k, j}^{K P}$. Now we need to prove that the $\tau$-function is given at once by $\frac{\operatorname{det} \varphi_{\gamma}\left(\lambda_{\delta}\right)}{\Delta(\lambda)}$ and $\operatorname{Det} \mathcal{H}_{i j}^{K P}\{t\}$. In order to compare these two 
expressions one should take $t_{k}=\frac{1}{k} \sum_{\gamma}^{n} \lambda_{\gamma}^{-k}$, so that

$$
\exp \left(\sum_{k>0} t_{k} z^{k}\right)=\prod_{\gamma=1}^{n} \frac{\lambda_{\gamma}}{\lambda_{\gamma}-z}=\left(\prod_{\gamma}^{n} \lambda_{\gamma}\right) \sum_{\gamma} \frac{(-)^{\gamma}}{z-\lambda_{\gamma}} \frac{\Delta_{\gamma}(\lambda)}{\Delta(\lambda)}
$$

where

$$
\Delta_{\gamma}(\lambda)=\prod_{\substack{\alpha>\beta \\ \alpha, \beta \neq \gamma}}\left(\lambda_{\alpha}-\lambda_{\beta}\right)=\frac{\Delta(\lambda)}{\prod_{\alpha \neq \gamma}\left(\lambda_{\alpha}-\lambda_{\gamma}\right)}
$$

and

$$
\left.\mathcal{H}_{i j}^{K P}\right|_{t_{k}=\frac{1}{k} \sum_{\gamma}^{n} \lambda_{\gamma}^{-k}}=\left(\prod_{\gamma}^{n} \lambda_{\gamma}\right) \sum_{\gamma} \frac{(-)^{\gamma+1} \Delta_{\gamma}(\lambda)}{\Delta(\lambda)} \lambda_{\gamma}^{i} \varphi_{-j}\left(\lambda_{\gamma}\right)
$$

As far as $n$ is kept finite, determinant of the infinite-size matrix (4.92), $\left.\operatorname{Det}_{i, j<0} \mathcal{H}_{i j}^{K P}\right|_{t_{k}=\frac{1}{k} \sum_{\gamma}^{n} \lambda_{\gamma}^{-k}}=0$ since it is obvious from (4.92) that the rank of the matrix is equal to $n$. Therefore let us consider the maximal non-vanishing determinant,

$$
\begin{gathered}
\left.\operatorname{Det}_{-n \leq i, j<0} \mathcal{H}_{i j}^{K P}\right|_{t_{k}=\frac{1}{k} \sum_{\gamma}^{n} \lambda_{\gamma}^{-k}}= \\
=\left(\prod_{\gamma}^{n} \lambda_{\gamma}\right)^{n} \operatorname{det}_{i \gamma}\left(\frac{(-)^{\gamma+1} \Delta_{\gamma}(\lambda)}{\lambda_{\gamma}^{i} \Delta(\lambda)}\right) \cdot \operatorname{det}_{\gamma j} \varphi_{j}\left(\lambda_{\gamma}\right)= \\
=\frac{\operatorname{det}_{\gamma j} \varphi_{j}\left(\lambda_{\gamma}\right)}{\Delta(\lambda)} .
\end{gathered}
$$

We used here the fact that determinant of a matrix is a product of determi- 
nants and reversed the signs of $i$ and $j$. Also used were some simple relations:

$$
\begin{gathered}
\prod_{\gamma=1}^{n} \frac{\Delta_{\gamma}(\lambda)}{\Delta(\lambda)}=\frac{1}{\Delta^{2}(\lambda)}, \\
\operatorname{det}_{i \gamma} \frac{1}{\lambda_{\gamma}^{i}}=\left(\prod_{\gamma}^{n} \lambda_{\gamma}\right)^{-1} \Delta(1 / \lambda), \\
\Delta(1 / \lambda)=\prod_{\alpha>\beta}\left(\frac{1}{\lambda_{\alpha}}-\frac{1}{\lambda_{\beta}}\right)=(-)^{n(n-1) / 2} \Delta(\lambda)\left(\prod_{\gamma}^{n} \lambda_{\gamma}\right)^{-(n-1)},
\end{gathered}
$$

thus

$$
\left(\prod_{\gamma}^{n} \lambda_{\gamma}\right)(-)^{n(n-1) / 2} \prod_{\gamma=1}^{n} \frac{\Delta_{\gamma}(\lambda)}{\Delta(\lambda)} \operatorname{det}_{i \gamma} \frac{1}{\lambda_{\gamma}^{i}}=\frac{1}{\Delta(\lambda)} .
$$

Since (4.93) is true for any $n$, one can claim that in the limit $n \rightarrow \infty$ we recover the statement, that $\tau^{K P}\{t\}=\operatorname{Det}_{i, j<0} \mathcal{H}_{i j}^{K P}$ with $\mathcal{H}_{i j}^{K P}$ given by eq. (4.92) (that formula does not refer directly to Miwa parametrization and is defined for any $t$ and any $j<0$ and $i$ ). This relation between $\varphi_{\gamma}$ 's and $\mathcal{H}_{i j}^{K P}$ can now be used to introduce negative times $\bar{t}_{k}$ according to the rule (4.58). Especially simple is the prescription for zero-time: $\mathcal{H}_{i j} \rightarrow \mathcal{H}_{i+N, j+N}$, when expressed in terms of $\varphi$ just implies that

$$
\frac{\operatorname{det} \varphi_{\gamma}\left(\lambda_{\delta}\right)}{\Delta(\lambda)} \rightarrow \frac{\operatorname{det} \varphi_{\gamma+N}\left(\lambda_{\delta}\right)}{(\operatorname{det} \Lambda)^{N} \Delta(\lambda)}
$$

Generalizations of (4.88), like

$$
\mathcal{H}_{i j}\{t, \bar{t}\}=\oint \oint z^{i} \bar{z}^{j}\langle 0|\psi(z) G \tilde{\psi}(\bar{z})| 0\rangle e^{\sum_{k}\left(t_{k} z^{k}+\bar{t}_{k} \bar{z}^{k}\right)} d z d \bar{z},
$$

also can be considered.

\subsection{Matrix models versus $\tau$-functions.}

We are now prepared to return to our main subject and discuss integrability properties of eigenvalue matrix models. The claim is that partition functions of all these models, when considered as functions of time-variables (parametrizing the shapes of potentials) are in fact $\tau$-functions of (perhaps, 
multicomponent) Toda-lattice and/or KP type. (Interesting non-eigenvalue models are believed to be related to integrable systems of more general type, not restricted to level $k=1$ Kac-Moody algebras.)

Partition functions are, however, not generic Toda or KP $\tau$-functions: first, they usually belong to some reduced hierarchies, second, the relevent operators $G$ (points of Grassmannian) are restricted to stay in peculiar domains of the universal module space, specified by "string equations". String equation is in fact nothing but the set of Ward-identities (Virasoro or $W$ constraints in the examples under investigation), which are now interpreted as equations on $G$. The very possibility of such interpretation is highly nontrivial and reflects some deep relation between the constraints and integrable structure. In the case of Virasoro constraints this is not a puzzle, because Virasoro algebra is a symmetry (covariance) of the hierarchy, the situation with other constraints is less clear (see the footnote at section 4.3). In fact, when applied to a $\tau$-function of appropriately reduced hierarchy, the infinitely many constraints usually become dependent and it is enough to impose only the lowest Virasoro constraint $L_{-1} \tau=0$ (or $\mathcal{L}_{-p} \tau=0$, where $p$ is the degree of reduction), in order to recover the entire set [29]. It is this lowest constraint (or rather its $t_{1}$-derivative, $\frac{\partial}{\partial t_{1}}\left(L_{-1} \tau\right)=0$ ) that traditionally carries the name of "string equation". It is often much simpler to deduce than the entire set of identites, what is important in practical applications (especially because determinant formulas, which imply integrability, can be also simpler to find in some situations than the Ward identities).

In order to give a complete description of some sort of (matrix) models from the point of view of integrability theory it is enough to specify the hierarchy, to which it belongs (if partition function is interpreted as $\tau$-function,

$$
Z_{\text {model }}\{t\}=\tau\left\{t \mid G_{\text {model }}\right\}
$$

and the string equation which serves to fix operator $G$ - the point in the universal module space. ${ }^{-5}$ After that it becomes an internal (yet unsolved) problem of integrability theory to explain, what is so special about the set of

\footnotetext{
37 As we argued in the Introduction and in section 2.1, the word "matrix" can be probably omited if generic Lagrangians are considered in other models of quantum field theory. Also the universal module space (where moduli are - of bundles over spectral Riemann surfaces) can (and should) be treated as a "space of theories". It is one of the great puzzles (and beauties) of string theory, that Riemann surfaces appear both in the world-sheet and spectral "dimensions". See [6] for more discussion on this issue.
} 
points $\left\{G_{\text {model }}\right\}$ in this space. (We shall touch this problem in the next subsection, devoted to Kac-Schwarz operators.) Alternatively, if there is nothing special, it is an (unsolved) problem of matrix model theory to find models, associated with any points $G$ in the universal module space (or explain what is an obstacle, if any).

We proceed now to description of particular matrix models from this point of view. As everywhere in these notes we consider only the most important classes of scalar-product, conformal (multicomponent) and Generalized Kontsevich models (GKM). All other examples (like models of complex, orthogonal, unitary etc matrices) can be included into consideration with more or less effort (see [28] and [91] for the cases of complex and unitary models respectively), but they do not add much for the general theory that we are now considering. String equations will be discussed in the next subsection.

Scalar-product models. These were exhaustively discussed in sections 3.53.7. We remind that all conventional multimatrix models (with inter-matrix interaction of the form $\exp \left(\operatorname{Tr} H^{(\alpha)} H^{(\alpha+1)}\right)$ belong to this class. The crucial formulas are:

$$
\begin{aligned}
Z_{N} & =\operatorname{Det}_{N} \mathcal{H}_{i j}^{f}=\operatorname{Det}_{0 \leq i, j \leq N-1} \mathcal{H}_{i j}^{f}= \\
& =\operatorname{Det}_{-N \leq i, j<0} \mathcal{H}_{i+N, j+N}^{f} ; \\
\mathcal{H}_{i j}^{f} & =\frac{\partial^{2}}{\partial t_{i} \partial t_{j}} \mathcal{H}^{f}=\left(\frac{\partial}{\partial t_{1}}\right)^{i}\left(\frac{\partial}{\partial t_{1}}\right)^{j} \mathcal{H}^{f} .
\end{aligned}
$$

Here

$$
\mathcal{H}_{i j}^{f}=\left\langle h^{i} \mid \bar{h}^{j}\right\rangle=\int d \hat{\mu}_{h, \bar{h}} e^{U(h)+\bar{U}(\bar{h})} h^{i} \bar{h}^{j} .
$$

Further,

$$
\begin{aligned}
e^{U(h)} & =e^{\sum_{k \geq 0} t_{k} h^{k}}=\sum_{l} h^{l} P_{l}(t) ; \\
e^{\bar{U}(\bar{h})} & =e^{\sum_{k \geq 0} \bar{t}_{k} \bar{h}^{k}}=\sum_{m} \bar{h}^{m} P_{m}(\bar{t})
\end{aligned}
$$

and thus

$$
\begin{aligned}
\mathcal{H}_{i j}^{f} & =\sum_{l, m}\left\langle\left\langle h^{i+l} \mid \bar{h}^{j+m}\right\rangle\right\rangle P_{l}(t) P_{m}(\bar{t})=\sum_{l, m} T_{l m}^{f} P_{l-i}(t) P_{m-j}(\bar{t}), \\
T_{l m}^{f} & =\left\langle\left\langle h^{l} \mid \bar{h}^{m}\right\rangle\right\rangle,
\end{aligned}
$$


where the scalar product $\langle\langle\mid\rangle\rangle$ is w.r.to the measure $d \hat{\mu}_{h, \bar{h}}$ (while $\langle\mid\rangle$ is w.r.to $\left.d \mu_{h, \bar{h}}=e^{U(h)+\bar{U}(\bar{h})} d \hat{\mu}_{h, \bar{h}}\right)$.

One would immediately recognize in these formulas representation (4.39) of Toda-lattice $\tau$-function, be there no additional restriction that determinant in (4.97) is over finite-dimensional $N \times N$ matrix (indices are constrained: $i, j \geq-N)$. This can be automatically taken into account if we require that

$$
T_{l m}^{f}=\infty \text { for all } l, m<0,
$$

and identify $Z_{N}$ as a $\tau$-function $\tau^{f}$ of forced Toda-lattice hierarchy (thus the supescript $f$ carried by $\mathcal{H}$ and $T$ ). We conclude that partition functions of any scalar-product model is a $\tau^{f}$-function of forced Toda-lattice hierarchy.

Let us now consider them as KP $\tau$-functions. This means that the $\bar{t}$-dependence is simply ignored. However, $N$ will be preserved explicitly as a parameter, labeling KP $\tau$-function. After Miwa transformation $t_{k}=$ $-\frac{1}{k} \sum_{\gamma} \lambda_{\gamma}^{-k}-r_{k}$, described in section 3.7, we get:

$$
Z_{N}=\hat{Z}_{N} \frac{\operatorname{det}_{\gamma \delta} \hat{Q}_{N+\gamma-1}\left(\lambda_{\delta}\right)}{\Delta(\lambda)}
$$

where $\hat{Q}$ are orthogonal polinomials w.r.to the measure $d \hat{\nu}_{h, \bar{h}}=e^{-\sum_{k} r_{k} h^{k}} d \hat{\mu}_{h, \bar{h}}$. We conclude that in the framework of KP hierarchy the scalar-product models are distinguished by the fact, that the corresponding $\varphi_{\gamma}(\lambda)$ in (4.86) are polinomials rather than infinite series in powers of $\lambda^{-1}$.

1-Matrix model. This is particular example of scalar product model with a local measure $d \mu_{h, \bar{h}}=e^{U(h)+\bar{U}(\bar{h})} \delta(h-\bar{h}) d h d \bar{h}$. In this case

$$
\mathcal{H}_{i j}^{f}=\left\langle h^{i} \mid \bar{h}^{j}\right\rangle=\left\langle h^{i+j}\right\rangle=\frac{\partial}{\partial t_{i+j}} \mathcal{H}^{f}=\left(\frac{\partial}{\partial t_{1}}\right)^{i+j} \mathcal{H}^{f} .
$$

Thus in this case we deal with the (forced) Toda-chain reduction of Todalattice hierarchy. In the end of this section 4 we use orthogonal polinomials to present a detailed description of 1-matrix models as a Toda-chain $\tau$-function.

This model can be alternatively defined as Gaussian Kontsevich model: see section 3.8. The fact that partition function is a $\tau$-function follows then from the general statement for GKM, see below. The fact that it is forced $\tau$-function is related to the property $c_{-N}=0$, mentioned at the end of s.3.8 
(and proved in s.3.9). Also reduction to Toda-chain hierarchy can be observed directly in terms of GKM: see ref. 36] for more details.

Multicomponent (conformal) matrix models. These are related to multicomponent hierarchies, with $\tau$-functions representable as correlators in multifermion systems. An example of determinant formula which substitutes (4.39) in the 2-component case, is given at the end of section 3.5, where it is derived from consideration of the relevant matrix model [39]. For derivation of the same determinant formula in the theory of $\tau$-functions see ref. 92. Generic theory of multicomponent hierarchies is now making its first steps and we do not review it in these notes. See [93] for the group-theory approach to the problem.

Generalized Kontsevich model. Determinant formulas for this case are derived in Section 3.3. The most important expression is

$$
Z_{V}\{N, T\}=\frac{1}{(\operatorname{det} \Lambda)^{N}} \frac{\operatorname{det}_{\gamma \delta} \varphi_{\gamma+N}\left(\lambda_{\delta}\right)}{\Delta(\lambda)},
$$

where

$$
\begin{aligned}
\varphi_{\gamma}(\lambda) & =\frac{1}{\sqrt{2 \pi}} e^{-\lambda V^{\prime}(\lambda)+V(\lambda)} \sqrt{V^{\prime \prime}(\lambda)} \int x^{\gamma-1} e^{-V(x)+V^{\prime}(\lambda) x} d x= \\
& =\lambda^{\gamma-1}\left(1+\mathcal{O}\left(\lambda^{-1}\right)\right)
\end{aligned}
$$

and

$$
\varphi_{\gamma}(\lambda)=\mathcal{A} \varphi_{\gamma-1}(\lambda)=\mathcal{A}^{\gamma-1} \Phi(\lambda) .
$$

For $N=0$ this is just the representation, peculiar for $\mathrm{KP} \tau$-function in Miwa parametrization $T_{k}=\frac{1}{k} \operatorname{tr} \Lambda^{-k}$, see eq.(4.86) above. Thus

$$
Z_{V}\{T\}=\tau^{K P}\left\{T \mid G_{V}\right\},
$$

where it is operator $G$ (the point in Grassmannian) which depends on the shape of potential $V(X)$. We also remind that the only way in which $Z$ depends on the size of the matrix $n$ is through the domain of variation of the time variables $T$. If (4.104) is extended to full Toda-lattice $\tau$-function, by introduction of negative times, we get [36]:

$$
\begin{gathered}
Z_{V}\{T, N, \bar{T}\}=\frac{\mathcal{C}_{V}^{-1}(\Lambda)}{(\operatorname{det} \Lambda)^{N}} e^{-\sum_{k>0} \bar{T}_{k} \operatorname{tr} \Lambda^{-k}} \times \\
\times \int_{n \times n} d X(\operatorname{det} X)^{N} \exp \left(-\operatorname{tr} V(X)+\operatorname{tr} \Lambda X+\sum_{k>0} \bar{T}_{k} \operatorname{tr} X^{-k}\right)
\end{gathered}
$$


When this extended partition function is considered as KP $\tau$-function, we have instead of (4.104):

$$
Z_{V}\{T, N, \bar{T}\}=\frac{1}{(\operatorname{det} \Lambda)^{N}} \frac{\operatorname{det}_{\gamma \delta} \varphi_{\gamma+N}^{\{\hat{V}\}}\left(\lambda_{\delta}\right)}{\Delta(\lambda)},
$$

and relevant $\varphi$-functions are

$$
\begin{aligned}
\varphi_{\gamma+N}^{\{\hat{V}\}}(\lambda) & =\frac{1}{\sqrt{2 \pi}} e^{-\lambda V^{\prime}(\lambda)+\hat{V}(\lambda)} \sqrt{V^{\prime \prime}(\lambda)} \int x^{\gamma-1} e^{-\hat{V}(x)+V^{\prime}(\lambda) x} d x= \\
& =\lambda^{N+\gamma-1}\left(1+\mathcal{O}\left(\lambda^{-1}\right)\right)
\end{aligned}
$$

with

$$
\begin{aligned}
\hat{V}(x) & \equiv V(x)-N \log x-\sum_{k>0} \bar{T}_{k} x^{-k}, \\
V(x) & =\hat{V}_{+}(x)
\end{aligned}
$$

(where $\hat{V}_{+}(x)$ is the positive-power fragment of Laurent series $\hat{V}(x)$ ). Functions $\varphi_{\gamma}(\lambda)$ in (4.105) are just equal to $\left.\varphi_{\gamma}^{\{\hat{V}\}}(\lambda)\right|_{\bar{T}=0}$.

\subsection{String equations and general concept of reduction}

The role of string equation is to fix the point $G$ in the universal module space (UMS), associated with the particular matrix model, so that partition function, considered as a function of time variables, will appear as the corresponding $\tau$-function of a fixed shape. In this sense the idea behind the string equation is exatly the same as reduction of integrable hierarchies. The difference is that linear reductions, as defined in the section 4.5 above, are not enough to fix $G$ unambiguously: they just specify certain subsets in the Grassmannian, which are still infinite-dimensional. The reason, why these are usually linear reductions that are considered in the conventional theory of integrable hierarchies, is that they are associated with the simplest possible Kac-Moody - subalgebras in the entire $G L(\infty)$. String equations, even their simplest examples, are usually fragments of more complicated - Virasoro and $W$-algebras, and are in fact considerably more restrictive. Moreover, string equation is usually a distinguished fragment, because it usually belongs to the Virasoro component of Ward identities, and Virasoro is still a Lie subalgebra in $G L(\infty)$. This is what makes the problem of string equations very similar to the "classical" one with linear reduction. 
More specifically, in order to include string equations (and in fact the entire set of Virasoro - but not $W$ - constraints) into consideration of reduction it is enough to allow the coefficients in (4.40) to depend on $t$ and $\bar{t}$, without changing the order of time-derivatives. Of course, there are no obvious reasons to think that any point $G$ in the UMS can be selected by imposing this kind of linear-derivative constraints on $\tau$-function, - and further investigation can require essential generalization of such restricted notion of string equation. However, some of the eigenvalue matrix models are already known to possess string equations of such simple type, associated with Virasoro subalgebras of $G L(\infty)$. We do not go into details of the general theory - it is far not completed yet, - but instead present several examples of how string equations arise in particular matrix models. These examples can illustrate also the simplifications arising when only string equations and not the entire sets of Ward identities need be derived. In particular, it is clear that in cases when $\tau$ is represented as $\operatorname{Det}_{i j} \mathcal{H}_{i j}$, a linear differential equation imposed on $\mathcal{H}_{i j}$ will give rise to a similar equation on $\tau$ itself. Most of known string equations can be derived with the help of this technical idea. They are usually associated with invariance of integrals under constant shifts of integration variables $\delta h=$ const in scalar-product and other discrete models, and with the action of operator $\operatorname{tr} \frac{\partial}{\partial L_{t r}}$ in GKM. For somewhat more involved ideas, associated with string equations see [94].

Scalar-product models. String equation can be easily deduced for very specific type of measures $d \hat{\mu}_{h, \bar{h}}$. Since integral

$$
\mathcal{H}_{i j}=\int h^{i} \bar{h}^{j} e^{U(h)+\bar{U}(\bar{h})} d \mu_{h, \bar{h}}
$$

is invariant under the shift of integration variable $\delta h=$ const,

$$
\int h^{i} \bar{h}^{j} e^{U(h)+\bar{U}(\bar{h})} d \hat{\mu}_{h, \bar{h}}\left[i h^{-1}+\frac{\partial U(h)}{\partial h}+\frac{\partial}{\partial h} \log \left(d \hat{\mu}_{h, \bar{h}}\right)\right]=0,
$$

or

$$
i \mathcal{H}_{i-1, j}+\sum_{k>0} k t_{k} \frac{\partial}{\partial t_{k-1}} \mathcal{H}_{i j}+\left[S\left(\frac{\partial}{\partial t}, \frac{\partial}{\partial \bar{t}}\right)\right]_{i j}=0
$$

String equation arises straightforwardly, when operator $S$ is linear. This is true, if $\log \left(d \hat{\mu}_{h, \bar{h}}\right) \sim h f(\hat{h})$ with any function $f(h)$. If the measure $d \hat{\mu}_{h, \bar{h}}$ is 
also required to be symmetric in $h$ and $\bar{h}$, we obtain conventional 2 -matrix model as the only example:

$$
d \hat{\mu}_{h, \bar{h}}=e^{c h \bar{h}} d h d \bar{h} .
$$

Equation for $\mathcal{H}_{i j}$ is:

$$
\left(\sum_{k>0} k t_{k} \frac{\partial}{\partial t_{k-1}}+c \frac{\partial}{\partial \bar{t}_{1}}\right) \mathcal{H}_{i j}=-i \mathcal{H}_{i-1, j}
$$

Its implication for $\hat{\tau}_{N}$ is:

$$
\left(\sum_{k>0} k t_{k} \frac{\partial}{\partial t_{k-1}}+c \frac{\partial}{\partial \bar{t}_{1}}\right) \hat{\tau}_{N}=0
$$

since the r.h.s. of (4.116) does not contribute to determinant (the entries in the $\mathrm{i}$-th row are proportional to those in the $i-1$-th row).

In particular case of 1-matrix model $c=0$, and we recognize the lowest Virasoro constraint $L_{-1} \hat{\tau}_{N}=0$. Traditionally the name of string equation is given not to $L_{-1}$-constraint itself, but to its $t_{1}$-derivative: $\frac{\partial}{\partial t_{1}}\left(L_{-1} \hat{\tau}_{N}\right)=0$. For 2-matrix model (4.117) is the lowest $(m=1, n=0)$ component of the Ward identities $\left(\tilde{W}_{n-m}^{(m+1)}(t)-(-)^{m+n} c^{n+1} \tilde{W}_{m-n}^{(n+1)}(\bar{t})\right) \hat{\tau}_{N}=0$. Of course, there is also a similar equation with $t \leftrightarrow \bar{t}$.

Multicomponent (conformal) models. The crucial feature of these models is that intermatrix interaction, when rewritten in terms of eigenvalues, usually contains only differences $h_{i}^{(\boldsymbol{\alpha})}-h_{j}^{(\boldsymbol{\beta})}$. Thus there is usually covariance under simultaneous shift of all eigenvalues $\delta h_{i}^{(\boldsymbol{\alpha})}=$ const by a same constant. This gives rise to a string equation of the form

$$
\left(\sum_{\boldsymbol{\alpha}} L_{-1}^{(\boldsymbol{\alpha})}\right) \tau_{\mathbf{N}}=0
$$

See 39] for details.

Generalized Kontsevich Model.

In order to derive string equation one should act on partition function $Z_{V}\left\{T_{k}=\frac{1}{k} \operatorname{tr} \Lambda^{-k}\right\}=\mathcal{C}_{V}^{-1} \mathcal{F}_{V}\left\{L=V^{\prime}(\Lambda)\right\}$ with operator $\operatorname{tr} \frac{\partial}{\partial L_{t r}}=\operatorname{tr} \frac{1}{V^{\prime \prime}(\Lambda)} \frac{\partial}{\partial \Lambda_{t r}}$. We can rewrite the result of this action in terms of time-derivatives,

$$
\operatorname{tr} \frac{\partial}{\partial L_{t r}} \log Z_{V}\{T\}=-\sum_{k>0}\left(\operatorname{tr} \frac{1}{V^{\prime \prime}(\Lambda) \Lambda^{k+1}}\right) \frac{\partial}{\partial T_{k}} \log Z_{V}\{T\} .
$$


Alternatively we can use the fact that $\operatorname{tr} \frac{\partial}{\partial L_{t r}}=\sum_{\gamma} \frac{1}{V^{\prime \prime}\left(\lambda_{\gamma}\right)} \frac{\partial}{\partial \lambda_{\gamma}}, l=V^{\prime}(\lambda)$, and explicit expression for $Z_{V}$ in terms of eigenvalues (Miwa coordinates),

$$
\begin{aligned}
& Z_{V} \sim e^{\operatorname{tr} V(\Lambda)-\operatorname{tr} \Lambda V^{\prime}(\Lambda)} \sqrt{\prod_{\gamma} V^{\prime \prime}\left(\lambda_{\gamma}\right)} \frac{\operatorname{det} \hat{\varphi}_{\gamma}\left(\lambda_{\delta}\right)}{\Delta(\lambda)} \sim \\
& \sim \frac{\operatorname{det} \varphi_{\gamma}\left(\lambda_{\delta}\right)}{\Delta(\lambda)},
\end{aligned}
$$

to get:

$$
\begin{gathered}
\left(\operatorname{tr} \frac{\partial}{\partial L_{t r}}\right) \log Z_{V}\{T\}= \\
=\frac{1}{2} \operatorname{tr} \frac{V^{\prime \prime \prime}(\Lambda)}{\left(V^{\prime \prime}(\lambda)\right)^{2}}+\frac{1}{2} \sum_{\gamma>\delta} \frac{V^{\prime \prime}\left(\lambda_{\gamma}\right)-V^{\prime \prime}\left(\lambda_{\delta}\right)}{\lambda_{\gamma}-\lambda_{\delta}} \cdot \frac{1}{V^{\prime \prime}\left(\lambda_{\gamma}\right) V^{\prime \prime}\left(\lambda_{\delta}\right)}- \\
-\operatorname{tr} \Lambda+\sum_{\beta} \frac{\partial}{\partial l_{\beta}} \log \operatorname{det}_{\gamma \delta} \hat{\varphi}_{\gamma}\left(l_{\delta}\right) .
\end{gathered}
$$

Comparison of these two expressions gives:

$$
\begin{gathered}
\frac{\mathcal{L}_{-1}^{(V)} Z_{V}}{Z_{V}} \equiv \frac{1}{Z_{V}}\left[\sum_{k>0}\left(\operatorname{tr} \frac{1}{V^{\prime \prime}(\Lambda) \Lambda^{k+1}}\right) \frac{\partial}{\partial T_{k}}+\right. \\
\left.+\frac{1}{2} \sum_{\gamma>\delta} \frac{V^{\prime \prime}\left(\lambda_{\gamma}\right)-V^{\prime \prime}\left(\lambda_{\delta}\right)}{\lambda_{\gamma}-\lambda_{\delta}} \cdot \frac{1}{V^{\prime \prime}\left(\lambda_{\gamma}\right) V^{\prime \prime}\left(\lambda_{\delta}\right)}-\frac{\partial}{\partial T_{1}}\right] Z_{V}= \\
=-\frac{\partial}{\partial T_{1}} \log Z_{V}+\operatorname{tr} \Lambda-\sum_{\beta} \frac{\partial}{\partial l_{\beta}} \log \operatorname{det}_{\gamma \delta} \hat{\varphi}_{\gamma}\left(l_{\delta}\right) .
\end{gathered}
$$

One can show that the r.h.s. is equal to zero, and thus the string equation arises in the form

$$
\mathcal{L}_{-1}^{(V)} Z_{V}=0 .
$$

If potential is monomial, $V_{p}=\frac{X^{p+1}}{p+1}$, then $r_{k}=-\frac{p}{p+1} \delta_{k, p+1}$ and

$$
\begin{gathered}
\mathcal{L}_{-1}^{V_{p}} \rightarrow \mathcal{L}_{-p} \equiv \\
\equiv \frac{1}{p}\left[\sum_{k>0}(k+p)\left(T_{k+p}+r_{k+p}\right) \frac{\partial}{\partial T_{k}}+\frac{1}{2} \sum_{k=1}^{p-1} k(p-k) T_{k} T_{p-k}\right] .
\end{gathered}
$$


The technical idea behind the proof [30] is to represent

$$
\frac{\partial}{\partial T_{1}} \log Z_{V}=\operatorname{Res} \frac{Z_{V}\left\{T_{k}+\frac{1}{k \lambda^{k}}\right\} d \lambda}{Z_{V}\left\{T_{k}\right\}}
$$

and make use of the second determinant representation in (4.120) both in the denominator and the numerator:

$$
\frac{\partial}{\partial T_{1}} \log Z_{V}=\operatorname{Res} \frac{d \lambda}{\prod_{\gamma=1}^{n}\left(\lambda-\lambda_{\gamma}\right)} \cdot \frac{\operatorname{det}\left(\begin{array}{cc}
\varphi_{\delta}\left(\lambda_{\gamma}\right) & \varphi_{n+1}\left(\lambda_{\gamma}\right) \\
\varphi_{\delta}(\lambda) & \varphi_{n+1}(\lambda)
\end{array}\right)}{\operatorname{det} \varphi_{\delta}\left(\lambda_{\gamma}\right)}
$$

Now we recall that

$$
\varphi_{\gamma}(\lambda) \sim \lambda^{\gamma-1}\left(1+\mathcal{O}\left(\lambda^{-1}\right)\right)
$$

At some moment we shall need even more: in fact

$$
\begin{aligned}
& \varphi_{\gamma}(\lambda) \sim \lambda^{\gamma-1}\left(1+\mathcal{O}\left(\lambda^{-2}\right)\right), \text { i.e. } \\
& \varphi_{\gamma}(\lambda)=\lambda^{\gamma-1}+c_{\gamma} \lambda^{\gamma-2}+\ldots, \text { and } c_{\gamma}=0 \text { for any } \gamma
\end{aligned}
$$

This is a rather delicate property of GKM, it follows from two facts: first, $\varphi_{1}=1+\mathcal{O}\left(\frac{V^{\prime \prime \prime \prime}}{\left(V^{\prime \prime}\right)^{2}}, \frac{\left(V^{\prime \prime \prime}\right)^{2}}{\left(V^{\prime \prime}\right)^{3}}\right)$, thus $c_{1}=0$, and second, Kac-Scwarz operator $\mathcal{A}$, defined in eq. (4.106) below, does not have contributions with zero-th power of $\lambda$, thus $c_{\gamma+1}=c_{\gamma}$. (For example, if $V(x)=\frac{x^{2}}{2}+a x, \varphi_{\gamma}(x)=$ $\frac{1}{\sqrt{2 \pi}} \int x^{\gamma-1} e^{-\frac{1}{2}(x-\lambda)^{2}} d x=\lambda^{\gamma-1}+0 \cdot \lambda^{\gamma-2}+\ldots$ the dangerous terms with $a$ simply do not show up in the expression for $\varphi_{\gamma}$.)

After this comment we can come back to evaluation of (4.126). The product in denominator, which arised from the Van-der-Monde determinant, is already proportional to $\lambda^{n}: \prod_{\gamma=1}^{n}\left(\lambda-\lambda_{\gamma}\right)=\lambda^{n}\left(1+\mathcal{O}\left(\lambda^{-1}\right)\right)$. Because of this and the asymptotic formulas (4.127), it is clear that if determinant in the numerator of (4.126) is rewritten as linear combination of $n \times n$ determinants with the coefficients $\varphi_{\gamma}(\lambda)$ from the last row, only items with $\gamma \geq n$ can contribute. There are two such items: $\gamma=n$ and $\gamma=n+1$. In the expansion of $(n+1) \times(n+1)$ determinant $\varphi_{n+1}(\lambda)$ is multiplied by det $\varphi_{\gamma}\left(\lambda_{\delta}\right)$, which exactly cancels with determinant in denominator, and the relevant 
contribution is

$$
\begin{array}{r}
\operatorname{Res} \frac{\varphi_{n+1}(\lambda) d \lambda}{\prod_{\gamma=1}^{n}\left(\lambda-\lambda_{\gamma}\right)}=c_{n+1}+\sum_{\gamma} \lambda_{\gamma}= \\
=c_{n+1}+\operatorname{tr} \Lambda .
\end{array}
$$

The item with $\varphi_{n}(\lambda)$ is

$$
\frac{\operatorname{det}\left(\varphi_{1}\left(\lambda_{\gamma}\right) \ldots \varphi_{n-1}\left(\lambda_{\gamma}\right) \varphi_{n+1}\left(\lambda_{\gamma}\right)\right)}{\operatorname{det}\left(\varphi_{1}\left(\lambda_{\gamma}\right) \ldots \varphi_{n-1}\left(\lambda_{\gamma}\right) \varphi_{n}\left(\lambda_{\gamma}\right)\right)} \operatorname{Res} \frac{\varphi_{n}(\lambda) d \lambda}{\prod_{\gamma=1}^{n}\left(\lambda-\lambda_{\gamma}\right)} \text {. }
$$

The remaining residue is just unity. Determinant in the numerator differs from the one in denominator by substitution of the colomn with entries $\varphi_{n}\left(\lambda_{\gamma}\right)$ for that with $\varphi_{n+1}\left(\lambda_{\gamma}\right)$.

At last we can return to eq.(4.122) and recall that $\frac{\partial}{\partial l} \hat{\varphi}_{\gamma}(l)=\varphi_{\gamma+1}(l)$, thus

$$
\begin{gathered}
\sum_{\beta} \frac{\partial}{\partial l_{\beta}} \log \operatorname{det}_{\gamma \delta} \hat{\varphi}_{\delta}\left(l_{\gamma}\right)= \\
\frac{\operatorname{det}\left(\hat{\varphi}_{1}\left(l_{\gamma}\right) \ldots \hat{\varphi}_{n-1}\left(l_{\gamma}\right) \hat{\varphi}_{n+1}\left(l_{\gamma}\right)\right)}{\operatorname{det}\left(\hat{\varphi}_{1}\left(l_{\gamma}\right) \ldots \hat{\varphi}_{n-1}\left(l_{\gamma}\right) \hat{\varphi}_{n}\left(l_{\gamma}\right)\right)}
\end{gathered}
$$

what is just the same as (4.130), since $\hat{\varphi}_{\delta}$ differ from $\varphi_{\delta}$ by $\delta$-independent factor of $e^{V(\lambda)-\lambda V^{\prime}(\lambda)} \sqrt{V^{\prime \prime}(\lambda)}$. Thus we conclude that the r.h.s. of (refsteqend) is equal to $-c_{n+1}$, which actually vanishes, as was explained several lines above.

Two things deserve paying attention in this derivation. First, it was absolutely crucial that we had $\frac{\partial}{\partial T_{1}} \log Z_{V}$ at the r.h.s. of (4.122) to make it vanishing, and therefore $\frac{\partial}{\partial T_{1}}$ immedeately appears in the expression for the $\mathcal{L}_{-1}^{(V)}$ operator at the 1.h.s. (this is the origin of $r_{k}$-corrections in (4.124). Second, the result is both simple and natural, but the proof is full of technical details and looks somewhat artificial. It becomes even more involved, when the general formula (4.136) for $T_{k}$-derivatives of $Z_{V}$ with $1 \leq k \leq p$ [40] is derived, which plays an important role in the theory of GKM and its applications to the theory of quantum gravity. The proof of the string equation is just a particular case of that formula, since using integral representation of $\hat{\varphi}(l)$ one can represent the r.h.s. of (4.131) as $\frac{1}{Z_{V}}\langle\operatorname{tr} X\rangle$, where \langle\rangle now stand for the average, defined by Kontsevich integral. Thus

$$
\mathcal{L}_{-1}^{(V)} Z_{V} \stackrel{(4.122)}{=}-\frac{\partial}{\partial T_{1}} Z_{V}+\langle\operatorname{tr} \Lambda-\operatorname{tr} X\rangle \stackrel{(4.136)}{=} 0 .
$$




\subsection{On the theory of GKM}

We remind that GKM is abbreviation for the Generalized Kontsevich model, This theory is the naturally broad collection of topics for a separate big section in these notes. However, we decided not to include such detailed presentation. This is because GKM theory seems too incomplete now. First, we believe that the natural invariant formulation - of which existing matrix integral is only a specific realization - is still lacking. Second, GKM is not yet generalized enough to fulfil its main purpose of incorporating infromation about all the models of $2 d$ gravity (in fact it should include even more: the entire theory of integrable hierarchies and geometrical quantization). Third, though the whole approach is very conceptual and deep, many proofs, as available nowadays, are still very technical and long. All this implies, that the proper view on the subject of GKM still needs to be found. At the moment we could describe two complementary approaches: one, starting from integral representations, another - from the Duistermaat-Heckman (localization) theory and Fourier analysis on group manifolds. Though intimately related, these two approaches are still technically different in too many respects. The second one is more fundamental (since ordinary integrals arise from discrete sums either in special limits or in the cases of inifinite-dimensional algebras, and, more important, since integral representation is only one of many possible ways to define the quantities of interest). However, many of the most important results, obtained in the first approach yet do not have their proper names and exact counterparts in the second one. We believe that this whole issue will be very much clarified in the near future and decided to postpone a detailed review till that time. What we can not avoid in these notes, is giving at least a list of topics, already included in the theory of GKM, and this is the purpose of the present subsection.

Kontsevich model with $V=\frac{X^{3}}{3}$ was derived by Maxim Kontsevich [22] from the original definition of topological $2 d$ gravity, given by E.Witten [9] in terms of generating functional for Chern classes of certain bundles over Riemann surfaces. Generalization of this reasoning (when more bundles are taken into consideration) leads to the theory of Landau-Ginzburg Gravity (LGG), which is believed to be the same as GKM, though not all the proofs are already avaliable. ${ }^{8}$

\footnotetext{
${ }^{38}$ Intermidiate results include the study of spherical approximation to LGG, which exhibits the structures, peculiar for "quasiclassical integrable hierarchies" (of which Bate-
} 
The crucial feature of non-perturbative partition functions, as we discussed at the neginning of section 2, is their intrinsic integrability. For $2 d$ gravity this general idea acquires a very concrete formulation: partition functions are usually just $\tau$-functions of conventional integrable hierarchies, moreover - for LGG, associated with minimal models, these are just ordinary multicomponent Toda hierarchies. ${ }^{39}$

M.Kontsevich found representation for generating functional in the form of matrix integral, i.e. formulated a matrix model, which later allowed to prove Witten's conjecture that the functional is in fact a $\tau$-function. The concept of GKM as a universal matrix model, including all the information about generic (eigenvalue?) matrix models and thus all the models of $2 d(?)$ gravity was introduced in [30], and the analogue of Kontsevich model with arbitrary potential $V(X)$, i.e. expression

$$
\begin{gathered}
\left.Z_{V}\{T\}\right|_{T_{k}=\frac{1}{k} \operatorname{tr} \Lambda^{-k}}=C_{V}(\Lambda)^{-1} \mathcal{F}_{V}\left(V^{\prime}(\Lambda)\right) \sim \\
\sim \frac{\sqrt{\operatorname{det} V^{\prime \prime}(\Lambda)}}{(2 \pi)^{n^{2} / 2} e^{\operatorname{tr}\left(\Lambda V^{\prime}(\Lambda)-V(\Lambda)\right)}} \int_{n \times n} d X e^{-\operatorname{tr} V(X)+\operatorname{tr} V^{\prime}(\Lambda) X}
\end{gathered}
$$

was proposed as an intemediate step in this direction. This (still restricted) version of GKM is already enough to unify all the $(p, 1)$-models of $2 d$ gravity. In some sense, $(p, q)$-models with $q \neq 1$ are also included, but in a very non-transparent way (using analytical continuation), which does not even explicitly respect the $p \leftrightarrow q$ symmetry. Partition function of such GKM, $Z_{V}\{T\}$ depends on two types of variables: time-variables $\hat{T}_{k}$ and potential $V$. Formally these two types of variables are absolutely different, $V$ being responsible for the choice of particular LGG model or, what is essentially the same, of particular reduction of Toda-lattice or KP hierarchy; while $\hat{T}_{k}$ are parameters of the generating functional of all correlation functions in this

man hierarchy to be briefly mentioned in section 5.2 below is an example), and which also arise in "quasiclassical approximation" to GKM. For some results in this direction see 95, 96, [97, ,17, 40, 41] and references therein.

${ }^{39}$ One can say that this is natural: both such models and Toda hierarchies are associated with the level $k=1$ Kac-Moody algebras and corresponding simplified versions of the WZNW model. However, too much still remains to be clarified about this "obvious" connection.

40 We remind that $\operatorname{det} V^{\prime \prime}(\Lambda)$ is defined somewhat tricky, see s.2.5 above. The same matrix integral (4.133) was also considered in refs. 38, 31, 32, 33. 
particular model. But of course, since we deal with exact (non-perturbative) approach, there is almost no real difference between these types of dependencies - on the model (vacuum state) and on the $\hat{T}$ 's: the model can be changed by non-infinitesimal shift of $\hat{T}$-variables. Technically, in GKM this is reflected in the identity of the form [40]:

$$
\mathcal{Z}_{V_{p}}\{T\}=f_{p}\left(r \mid \hat{T}_{k}+r_{k}\right) \cdot \tau\left\{\hat{T}_{k}+r_{k} \mid G_{p}\right\}
$$

where $r_{k}=\frac{p}{k(p-k)} \operatorname{Res}\left(V^{\prime}(\mu)\right)^{1-\frac{k}{p}} d \mu$ provide a specific parametrization of potentials $V$, which is here assumed to be any polinomial of degree $p$, and $f_{p}$ is some simple function:

$$
\begin{array}{r}
f_{p}\left(r \mid \hat{T}_{k}+r_{k}\right)=\exp -\frac{1}{2} \sum_{i, j} A_{i j}(r)\left(\hat{T}_{i}+r_{i}\right)\left(\hat{T}_{j}+r_{j}\right) \\
A_{i j}=\operatorname{Res}\left(V^{\prime}(\mu)\right)^{i / p} d\left(V^{\prime}(\mu)\right)^{j / p}=\frac{\partial^{2} \log \tau_{0}^{(p)}}{\partial t_{i} \partial t_{j}}
\end{array}
$$

and $\tau_{0}^{(p)}$ is a $\tau$-function of "quasiclassical hierarchy". What is important, $G_{p}$ (which define the shape of the $\tau$-function as function of $\hat{T}+r$ ) and $f_{p}$ depend only on the degree $p$, but not on the other details of the shape of the potential. This is a deep formula. It accounts for two phenomena at once: First, it says that $Z$ depends on the sum of $\hat{T}$ and $r$.t Second, dependence on $V$ is not quite smooth: when the degree of potential changes, the shapes of the functions $f$ and $\tau$ also changes abruptly. Another side of the same phenomenon is that partition function $Z_{V}\{T\}$, which in principle is well defined as a matrix integral for all choices of $V$ and $L$ (thus $\hat{T}$ ) at once, is in fact singular at some points: there are phase transitions, manifesting the switch

41 In Miwa parametrization $\hat{T}_{k}=\frac{1}{k} \operatorname{tr}\left(V_{p}^{\prime}(\Lambda)\right)^{-k / p}$. Throughout these notes we used different time-variables $T_{k}=\frac{1}{k} \operatorname{tr} \Lambda^{-k}$, which are independent of the potential $V$, instead $V$-dependence of $Z_{V}$ - which we did not really study - was rather nontrivial. If expressed in terms of $\hat{T}$, partition function $\hat{Z}_{V}\{\hat{T}+r\}=Z_{V}\{T\}$ becomes almost independent of $V$ : it changes - abruptly - only when the degree $p$ of potential changes. This second type of description is of course in better accordance with the symmetries of particular model - which are different in different "vacua" (for different $p$ ). Therefore these are variables $\hat{T}+r$, rather than in $T$ which arise naturally in Ward identites - as we saw in sections 2.5 and 2.6. T's and $\hat{T}$ 's are suited for different purposes: $T$ 's are nice when universality aspects of GKM are concerned, while $\hat{T}$ 's arise when specific features of particular models (orbits, vacua) are considered. 
from one LGG model to another. After a phase transition original integral expression becomes sonewhat symbolical: it defines partition function only in the sense of analytical continuation, and it is a separate problem to find an integral representation, adequate in the new phases. In practice, what is nicely decribed by the integral representation GKM in the form of eq. (4.133), are $(p, 1)$-models, with $p+1$ being just the power of potential $V(x)$. What is not yet found, is analogous representation for $(p, q)$-models with $q \neq 1$ (it can involve multiple matrix integrals, and universal model is supposed to be "matrix quantum mechanics in external fields").

Derivation of the crucial formula (4.134)in any approach - starting from GKM in the form of either LGG or matrix integrals - is still very tedious. In matrix-model representation it relies upon identity 40

$$
\begin{gathered}
\frac{\partial Z_{V}}{\partial T_{k}}=\left\langle\operatorname{tr} \Lambda^{k}-\operatorname{tr} X^{k}\right\rangle \equiv \\
\equiv \mathcal{C}_{V}^{-1} \int\left(\operatorname{tr} \Lambda^{k}-\operatorname{tr} X^{k}\right) e^{-\operatorname{tr} V_{p}(X)+\operatorname{tr} V_{p}^{\prime}(\Lambda) X} d X \quad \text { for } 1 \leq k \leq p,
\end{gathered}
$$

which look trivial but are rather hard to derive. (A proof of the string equation in GKM at the end of the previous subsection is the simplest example of this kind of exersices.) Certainly some simple derivation "in two lines" should exist, but it is not yet found. Formulas of this kind are very important for all aspects of GKM theory. Besides other things, they are just necessary to actually evaluate correlation functions in $(p, 1)$-models of $2 d$ gravity, of which $Z_{V}\{T\}$ is a generating functional. If instead of these "physical" questions, one asks about integrability theory, identites of this sort also play important role. For example, looking at (4.136) for a special $k=p$ and special choice of potential - monomial $V_{p}(X)=\frac{X^{p+1}}{p+1}$,- ane can note that the r.h.s. vanishes: this is just a Ward identity, reflecting invariance under the shift of the integration variable, $\delta X=$ const. This is a simplest version of a more general statement:"t2

$$
\text { if } V_{p}(X)=\frac{X^{p+1}}{p+1}, \quad \text { then } \quad \frac{\partial Z_{V}}{\partial T_{p k}}=0 \text { for all } n \in Z_{+} .
$$

42 This property was technically implicit in original Kontsevich's work [22] for $p+1=3$, where it was related with certain combinatorial identities. A tricky proof, relying upon properties of $\tau$-functions, was given for any $p$ in [30]. An example of straightforward proof, again for $p+1=3$, - just in terms of Kontsevich matrix integrals - can be found in ref. 99. 
Looking from the point of view of integrable hierarchies, one immediately recognizes (4.137) as an example of reduction condition (4.40). It corresponds to the so called $p$-reduction of KP-hierarhy, of which $\operatorname{KdV}(p=2)$ and Boussinesq $(p=3)$ are the most celebrated examples. We refer to 30 and [40] for all details and references, the only thing to mention here is that the slightly weaker verison of the constraint (4.137),

$$
\frac{\partial Z_{V}}{\partial T_{p n}}=a_{n}=\text { const }
$$

where $a_{n}$ do not depend on any time variables, can be simply expressed in Miwa parametrization: it is just the statement that $\varphi$-functions in

$$
Z_{V}=\frac{\operatorname{det}_{\gamma \delta} \varphi_{\gamma}\left(\lambda_{\delta}\right)}{\Delta(\lambda)}
$$

satisfy the $p$-reduction condition:

$$
\lambda^{p} \varphi_{\gamma}(\lambda)=\sum_{\delta=1}^{\gamma+p} \hat{\mathcal{V}}_{\gamma \delta} \varphi_{\delta}(\lambda)
$$

This a restrictive relation, because $\varphi$ 's are infinite seria in $1 / \lambda$, while at th r.h.s. in (4.139) there is only a finite number of items. In GKM it is satisfied for monomial potential just as a corollary of Gross-Newman equation, or more exact, of the Ward identity for the integral

$$
\varphi_{\gamma}(\lambda) \sim \int x^{\gamma-1} e^{-V(x)+V^{\prime}(\lambda) x} d x
$$

Indeed, integral does not change under the shift $\delta x=$ const, and this implies:

$$
\int x^{\gamma-1}\left(V^{\prime}(x)-V^{\prime}(\lambda)-\frac{\gamma-1}{x}\right) e^{-V(x)+V^{\prime}(\lambda) x} d x=0,
$$

i.e.

$$
\sum_{k=1}^{p+1} k v_{k}\left(\varphi_{\gamma+k-1}(\lambda)-\lambda^{k-1} \varphi_{\gamma}(\lambda)\right)-(\gamma-1) \varphi_{\gamma-1}=0 .
$$

If only $v_{p+1} \neq 0$, this leads to an identity of the required form of (4.139). This description of reduction can be modified to allow for non-monomial potentials, making use of the concept of "equivalent hierarchies", see [100], [40]: 
in this framework the reduction condition is

$$
V^{\prime}(\lambda) \varphi_{\gamma}(\lambda)=\sum_{\delta} \mathcal{V}_{\gamma \delta} \varphi_{\delta},(\lambda)
$$

but classes of essentially different reductions are labeled by the degree of potential only.

As we already discussed in the previous subsection, linear constraints like (4.139) are not restrictive enough to fix the shape of the $\tau$-function (the point $G$ in the universal module space) unambigously: string equation should be also imposed. If expressed in terms of $\varphi$ 's, string equation is just the property (4.106):

$$
\varphi_{\gamma+1}=\mathcal{A} \varphi_{\gamma}
$$

where the Kac-Schwarz operator

$$
\mathcal{A}=\frac{1}{V^{\prime \prime}(\lambda)} \frac{\partial}{\partial \lambda}-\frac{1}{2} \frac{V^{\prime \prime \prime}(\lambda)}{\left(V^{\prime \prime}(\lambda)\right)^{2}}+\lambda .
$$

It has obvious generalization of the form

$$
\mathcal{A}_{p, q}=\frac{\partial}{\partial V_{p}^{\prime}(\lambda)}-\frac{1}{2} \frac{V_{p}^{\prime \prime \prime}(\lambda)}{\left(V_{p}^{\prime \prime}(\lambda)\right)^{2}}+Q_{q}^{\prime}(\lambda),
$$

where $Q_{q}(\lambda)$ is a polinomial of degree $q+1$ and (4.142) is substituted be

$$
\varphi_{\gamma+q}=\mathcal{A}_{p, q} \varphi_{\gamma}
$$

This generalization is naturally related to the string equation in $(p . q)$ models, see 41] and references therein. Generic $(p, q)$ LGG model can be described by a system of constraints,

$$
\begin{aligned}
\left(\lambda^{p}-\mathcal{V}_{p}\right)\{\varphi\} & =0, \\
\mathcal{A}_{p, q}\{\varphi\} & =0,
\end{aligned}
$$

where both operators $\mathcal{V}_{p}$ and $\mathcal{A}_{p, q}$ are not uniquely fixed by choosing $p$ and $q$, and there is also a freedom to change variables $\lambda \rightarrow f(\lambda)$ and make a triangular transformation of basis $\varphi_{\gamma} \rightarrow \varphi_{\gamma}+\sum_{\delta<\gamma} C_{\gamma \delta} \varphi_{\delta}$. Altogether the set of equations (4.146) modulo these allowed transformations is finite - 
$(p-1)(q-1)$ - dimensional, this is dimension of the module space of LGG models with given $p$ and $q$. Kontsevich integral can be now used to establish duality transformation from $(p, q)$ to $(q, p)$ model [41]:

$$
Z_{V, Q}(\Lambda)=C_{V, Q}^{-1}(\Lambda) \int_{n \times n} d X e^{-\operatorname{tr} S_{V, Q}(X, \Lambda)+\operatorname{tr} V^{\prime}(\Lambda) Q^{\prime}(X)} Z_{Q, V}(X) .
$$

Here

$$
S_{V, Q}(x, \lambda)=\int^{x} V^{\prime}(y) Q^{\prime \prime}(y) d y=\int^{x} V^{\prime}(y) d Q^{\prime}(y),
$$

As usual, $C_{V, Q}(\Lambda)$ is the quasiclassical approximation to the integral, and $Z_{V, Q}(\Lambda) \equiv \frac{\operatorname{det}_{\gamma \delta} \varphi_{\gamma}\left(\lambda_{\delta}\right)}{\Delta(\lambda)}$, where $\varphi$ are solutions to 4.146 with $\mathcal{V}_{p}$ and $\mathcal{A}_{p, q}$ defined by eqs.(4.141) and (4.144) respectively. This relation does not provide any formula for $Z_{V_{p}, Q_{q}}(\Lambda)$ unless $q=1$. The case of $q=1$ is distinguished because $Z_{Q_{1}, V_{p}}$ is trivial. Indeed, the 1-reduction constraint $\lambda \varphi_{\gamma}=\varphi_{\gamma+1}+\sum_{\delta \leq \gamma} \mathcal{V}_{\gamma \delta} \varphi_{\delta}$, implies that $\operatorname{det}_{\gamma \delta} \varphi_{\gamma}\left(\lambda_{\delta}\right)=\Delta(\lambda) \prod_{\delta} \varphi_{1}\left(\lambda_{\delta}\right)$, thus $Z_{Q_{1}, V_{p}}=\exp \sum_{k} a_{k} T_{k}$, what is essentially the same as $Z_{Q_{1}, V_{p}}=1$, tha and (4.147) is just our old formula (4.133) for the $(p, 1)$ version of GKM. (In fact $Q_{1}(X) \sim X^{2}$, and $Z_{Q_{1}, V_{p}}$ is nothing but Gaussian Kontsevich model. It is trivial when the "zero-time" $N=0$, as we assume here.) Matrix model realization of $Z_{V_{p}, Q_{q}}$ for $q \neq 1$ is yet unknown.

This is not the only important further generalization of GKM (4.133). Another one is implied by the formula for $\mathcal{F}_{V}$ in terms of eigenvalues from the section 3.3,

$$
\mathcal{F}_{V} \sim \prod_{\gamma=1}^{n} \int d x_{\gamma} e^{-V\left(x_{\gamma}\right)} \Delta^{2}(x) I(x, l) .
$$

43 Also expression for $r_{k}$-variables is now modified:

$$
r_{k}=\frac{p}{k(p-k)} \operatorname{Res}\left(V_{p}^{\prime}(\mu)\right)^{1-k / p} d Q_{p}^{\prime}(\mu)
$$

For monomial $V_{p}$ and $Q_{q} r_{k}=-\frac{p}{p+q} \delta_{k, p+q}$.

44 Since $\varphi_{1}(\lambda)=1+\sum_{k>0} b_{k} \lambda^{-k}, \quad \log \varphi_{1}(\lambda)=\sum_{k>0} \frac{a_{k}}{k} \lambda^{-k}$, and the sum $\sum_{\delta} \log \varphi_{1}\left(\lambda_{\delta}\right)=\sum_{k>0} \frac{a_{k}}{k}\left(\sum_{\delta} \lambda_{\delta}^{-k}\right)=\sum_{k>0} a_{k} T_{k}$. Addition of any linear combination of time-variables to $\log \tau$ does not essentially change $\tau$-function. For example, the ordinary integrable equations (like $\mathrm{KdV}$ or $\mathrm{KP}$ ) are usually written in terms of variables like $u=\frac{\partial^{2}}{\partial T_{1}} \log \tau$, which are second derivatives of $\log \tau$. 
As it was already mentioned in section 3.3, the Itzykson-Zuber integral

$$
I(x, l) \sim \int[D U] e^{\operatorname{tr} U X U^{\dagger} L} \sim \frac{\operatorname{det}_{\gamma \delta} e^{x_{\gamma} l \delta}}{\Delta(x) \Delta(l)}
$$

is in fact a coadjoint orbit integral and has group theoretical interpretation: under certian conditions it turns into a character $\chi_{R}(g)=\operatorname{Tr}_{R} g$ of the group $G L(n)$. Here $g \equiv e^{L}$ is considered as a group element, representation $R$ is labeled by integer-valued parameters $m_{1}, \ldots, m_{n}$ - essentially the lengths of rows in the Young diagramm. Exact statement is:

$$
I(m, l) \cdot \frac{\Delta(l)}{\Delta(g)}=\frac{\operatorname{det}_{\gamma \delta} g_{\gamma}^{m_{\delta}}}{\Delta(m) \Delta(g)}=\frac{\chi_{R}(g)}{d_{R}},
$$

i.e. in order to get a character we should integrate over matrices $X$ with integer-valued eigenvalues.t.0 Dimension $d_{R}$ of representation can be also expressed in terms of $m$-variables: $d_{R}=\Delta(m)$. As to the traces $\operatorname{tr} X^{k}=$ $\sum_{\gamma} x_{\gamma}^{k} \rightarrow \sum_{\gamma} m_{\gamma}^{k}$, which appear in the action of GKM, they are very similar to the $k$-th Casimir eigenvalue $C_{k}(R)$ (though is not exactly the same). Thus we see that the integral in (4.149) is in fact very similar to

$$
\mathcal{F}_{V}^{q u}\{g, \bar{g}\} \equiv \sum_{R} \chi_{R}(\bar{g}) \chi_{R}(g) e^{-\sum_{k=0}^{\infty} v_{k} C_{k}(R)}
$$

evaluated at the point $\bar{g}=I$. The only real difference is that instead of the integral we have a sum over discrete values of $m$ (sum over all the representations, or a model of $G L(n)$ ). This "discretized" (quantum?) GKM is more general that the continuum one which can be obtained by various limiting procedures. It is now obvious that the theory of discretized GKM largely overlaps with that of $2 d$ Yang-Mills theory. The simplest ingredient

\footnotetext{
45 The ratio
}

$$
\frac{\Delta(l)}{\Delta(g)}=\prod_{\gamma>\delta} \frac{l_{\gamma}-l_{\delta}}{e^{l_{\gamma}}-e^{l_{\delta}}}
$$

is the usual correction factor, which is the price for the possibility to reduce quantummechanical problem of motion on the orbit to a single matrix integral. The full problem of matrix quantum mechanics can and should be considered as a multi-matrix (in fact, infinite-matrix) generalization of GKM (4.133), which incorporates all the $(p, q)$ LGG models. 
of this theory is the classical result [101], that $G L(N)$ characters are in fact (singular) Toda-lattice and KP $\tau$-functions. Moreover, the entire sum at the r.h.s. of (4.152), if considered as a function of $T_{k}=\frac{1}{k} \operatorname{tr} g^{k}, \bar{T}_{k}=\frac{1}{k} \operatorname{tr} \bar{g}^{k}$ is in fact a Toda-lattice $\tau$-function. There are also features parallel to (4.134). We refer to 102 for a little more details about discretized GKM (see also a recent paper [81]). This is one more very important direction of the further investigation of GKM.

\subsection{1-Matrix model versus Toda-chain hierarchy}

At the end of this section we use an explicit example of dicrete 1-matrix model [26] to illustrate, how a more familiar Lax description of integrable hierarchies arises from determinant formulas. This example will be also usefull in Section 5.3 below, when one of the ways to take double-scaling continuum limit of the 1-matrix model will be discussed. Lax representation appears usually after some coordinate system is chosen in the Grassmannian. In the example which we are now considering this system is introduced by the use of orthogonal polinomials.

We already know from section 3.6, that partition function of 1-matrix model (which is a one-component model) is given by

$$
Z_{N}=\operatorname{Det}_{0<i, j \leq N}\left\langle h^{i} \mid h^{j}\right\rangle=\prod_{i=0}^{N-1} e^{\phi_{i}}=Z_{1} \prod_{i=1}^{N-1} R_{i}^{N-i}
$$

where the last two representations are in terms of the norms of orthogonal polinomials

$$
\left\langle Q_{n} \mid Q_{m}\right\rangle=e^{\phi_{n}} \delta_{n m}
$$

and parameter of the 3 -term relation

$$
\begin{gathered}
h Q_{n}(h)=Q_{n+1}(h)+c_{n} Q_{n}(h)+R_{n} Q_{n-1}(h), \\
Z_{1}=e^{\phi_{0}}=\langle 1 \mid 1\rangle, \quad R_{n}=e^{\phi_{n}-\phi_{n-1}} .
\end{gathered}
$$

Of course all the information is contained in the determinant formula together 
with the rule, which defines time-dependence of $\mathcal{H}_{i j}^{f}=\left\langle h^{i} \mid h^{j}\right\rangle=\hat{\mathcal{H}}_{i+j}^{f}$ :

$$
\begin{gathered}
\frac{\partial \mathcal{H}_{i j}^{f}}{\partial t_{k}}=\mathcal{H}_{i+k, j}^{f}=\mathcal{H}_{i, j+k}^{f}, \quad \text { or } \\
\frac{\partial \hat{\mathcal{H}}_{i}^{f}}{\partial t_{k}}=\hat{\mathcal{H}}_{i+k}^{f} .
\end{gathered}
$$

(The possibility to express everything in terms of $\mathcal{H}_{i}^{f}$ with a single matrix index $i$ is the feature of Toda-chain reduction of generic Toda-lattice hierarchy.)

However, in order to reveal the standard Lax representation we need to go into somewhat more involved considerations. Namely, we consider representation of two operators in the basis of orthogonal polinomials. First,

$$
h^{k} Q_{n}(h)=\sum_{m=0}^{n+k} \frac{\left\langle n\left|h^{k}\right| m\right\rangle}{\langle m \mid m\rangle} Q_{m}(h)=\sum_{m=0}^{n+k} \gamma_{n m}^{(k)} Q_{m}(h)
$$

(here the simplified notation is introduced for $\langle n|f(h)| m\rangle \equiv\left\langle Q_{n}|f(h)|\right.$ $\left.Q_{m}\right\rangle$ and $\gamma_{n m}^{(k)} \equiv \frac{\left\langle n\left|h^{k}\right| m\right\rangle}{\langle m \mid m\rangle}$.) Second,

$$
\begin{gathered}
\frac{\partial Q_{n}(h)}{\partial t_{k}}=-\sum_{m=0}^{n-1} \frac{\left\langle n\left|h^{k}\right| m\right\rangle}{\langle m \mid m\rangle} Q_{m}(h)=-\sum_{m=0}^{n-1} \gamma_{n m}^{(k)} Q_{m}(h), \\
\frac{\partial \phi_{n}}{\partial t_{k}}=\frac{\left\langle n\left|h^{k}\right| n\right\rangle}{\langle n \mid n\rangle}=\gamma_{n n}^{(k)} .
\end{gathered}
$$

(These last relations arise from differentiation of orthogonality condition (4.154):

$$
\begin{gathered}
e^{\phi_{n}} \frac{\partial \phi_{n}}{\partial t_{k}} \delta_{n m}=\frac{\partial\left\langle Q_{n} \mid Q_{m}\right\rangle}{\partial t_{k}}= \\
=\left\langle\frac{\partial Q_{n}}{\partial t_{k}} \mid Q_{m}\right\rangle+\left\langle Q_{n} \mid \frac{\partial Q_{m}}{\partial t_{k}}\right\rangle+\left\langle Q_{n}\left|h^{k}\right| Q_{m}\right\rangle
\end{gathered}
$$

by looking at the cases of $m<n$ and $m=n$ respectively.)

From these relations one immediately derives the Lax-like formula:

$$
\frac{\partial \gamma_{n m}^{(k)}}{\partial t_{q}}=-\sum_{l=m-k}^{n-1} \gamma_{n l}^{(q)} \gamma_{l m}^{(k)}+\sum_{l=m+1}^{n+k} \gamma_{n l}^{(k)} \gamma_{l m}^{(q)}
$$


or, in a matrix form,

$$
\frac{\partial \gamma^{(k)}}{\partial t_{q}}=\left[R \gamma^{(q)}, \gamma^{(k)}\right]
$$

where

$$
R \gamma_{m n}^{(k)} \equiv\left\{\begin{array}{c}
-\gamma_{m n}^{(k)} \text { if } m>n \\
\gamma_{m n}^{(k)} \text { if } m<n
\end{array}\right.
$$

(We remind that usually $R$-matrix acts on a function $f(h)=\sum_{n=-\infty}^{+\infty} f_{n} h^{n}$ according to the rule: $R f(h)=\sum_{n \geq l} f_{n} h^{n}-\sum_{n<l} f_{n} h^{n}$ with some "level" l.) These $\gamma^{(k)}$ are not symmetric matrices, but one can also rewrite all the formulas above in terms of symmetric ones:

$$
\mathcal{L}_{m n}^{(k)} \equiv e^{\frac{1}{2}\left(\phi_{n}-\phi_{m}\right)} \gamma_{m n}^{(k)}=\frac{\left\langle m\left|h^{k}\right| n\right\rangle}{\sqrt{\langle m \mid m\rangle\langle n \mid n\rangle}}
$$

From eqs.41.158) one can easily deduce Toda-equations for $\phi_{n}$ :

$$
\begin{gathered}
\frac{\partial^{2} \phi_{n}}{\partial t_{k} \partial t_{l}}=\frac{\partial}{\partial t_{k}} \frac{\left\langle n\left|h^{l}\right| n\right\rangle}{\langle n \mid n\rangle}= \\
=\left(\sum_{m>n}-\sum_{m<n}\right) \frac{\left\langle n\left|h^{k}\right| m\right\rangle\left\langle m\left|h^{l}\right| n\right\rangle}{\langle m \mid m\rangle\langle n \mid n\rangle},
\end{gathered}
$$

where the r.h.s. can be expressed in terms of $R_{m}=e^{\phi_{m}-\phi_{m-1}}$. In particular,

$$
\frac{\partial^{2} \phi_{n}}{\partial t_{1} \partial t_{1}}=R_{n+1}-R_{n}=e^{\phi_{n+1}-\phi_{n}}-e^{\phi_{n}-\phi_{n-1}} .
$$

Let us also mention that in this formalism the Ward identities (Virasoro constraints) follow essentially from the relation

$$
\left(\frac{\partial}{\partial h}\right)^{\dagger}=-\frac{\partial}{\partial h}-\sum_{k>0} k t_{k} h^{k-1}
$$

where Hermitean conjugation is w.r.to the scalar product $\langle\mid\rangle$. For example, this relation implies, that

$$
\left\langle Q_{n} \mid \frac{\partial Q_{n}}{\partial h}\right\rangle=-\left\langle\frac{\partial Q_{n}}{\partial h} \mid Q_{n}\right\rangle-\sum_{k>0} k t_{k}\left\langle Q_{n}\left|h^{k-1}\right| Q_{n}\right\rangle .
$$


Now we note that $\frac{\partial Q_{n}}{\partial h}$ is a polinomial of degree $n-1$, thus $\left\langle Q_{n} \mid \frac{\partial Q_{n}}{\partial h}\right\rangle=0$. (In fact

$$
\left.\frac{\partial Q_{n}}{\partial h}=-\sum_{k>0} k t_{k}\left(\sum_{m=0}^{n-1} \gamma_{n m}^{(k-1)} Q_{m}\right)=-\sum_{k>0} k t_{k} \frac{\partial Q_{n}}{\partial t_{k-1}} .\right)
$$

Also we recall that $\left\langle Q_{n}\left|h^{k-1}\right| Q_{n}\right\rangle=\left\langle Q_{n} \mid Q_{n}\right\rangle \frac{\partial \phi_{n}}{\partial t_{k-1}}$, and obtain:

$$
\sum_{k>0} k t_{k} \frac{\partial \phi_{n}}{\partial t_{k-1}}=0
$$

for any $n$. This should be supplemented by relation $\frac{\partial \phi_{n}}{\partial t_{0}}=\phi_{n}$. In order to get the lowest Virasoro constraint (string equation), $L_{-1} Z_{N}=0$ or $L_{-1} \log Z_{N}=$ 0 it is enough just to sum over $n$ from 0 to $N-1$.

For more details about 1-matrix model, Toda-chain hierarchy and application of the formalism of orthogonal polinomials in this context see [26].

\section{Continuum limits of discrete matrix mod- els}

\subsection{What is continuum limit}

Continuum limit of matrix models is, of course, the crucial issue for their physical applications whenever these models are interpreted as discrete (lattice) approximations to continuum theory. The very first thing to be kept in mind is that it is not the only possible view on matrix models. Another approach considers them as describing topological (and thus also in a certain sense "discrete") properties of the theory. Such models, when appearing in the field of, say, quantum gravity (which after all is a sort of a pure topological theory) do not require any continuum limit to be taken: their discrete nature (occurence of integer-valued matrix indices) reflects not the discrete approximation to the space-time (which does not really exist in quantum gravity), but rather the essential discreteness of the underlying structures: topology of the module spaces of geometries. Example of matrix models which allow for 
this kind of interpretation - in terms of topology of module spaces of bundles over Riemann surfaces - is provided by Kontsevich models, and this is why they usually do not require any continuum limit and why we once called them "continuous matrix models" in the Introduction to these notes. The models which are usually interpreted in more traditional way - as lattice theories - are represented by our "discrete" models, the 1-matrix, conventional and "conformal" multimatrix models being included into this class. More sophisticated examples are provided by " $c=1 "$-theories, Kazakov-Migdal model and, say, Wilson's QCD (and infinetely many other lattice theories). It is not a surprise that continuum limits of some discrete models provide the theories of Kontsevich type: this happens whenever continuum theory is supposed to have a kind of topological nature. This is usually the case for quantum gravity (which, as we said, is conceptually a topological theory in the "module space of geometries" - the notion which is already made more or less explicit in the $2 d$ case), but in principle this can be also true for many other theories, including exhaustive quantum theory of Yang-Mills fields (again there is already considerable progress in this direction, as soon as $2 d$ Yang-Mills model is concerned). There should not be confusion about the presence of gauge particles in dimensions greater than 2 (for Yang-Mills) and 3 (for gravity): there is no reason to prevent generic topological theory from possessing continuum spectrum of excitations, though explicit analogue of Kontsevich-like description of such situations is not yet found (as we mentioned many times, it should probably rely upon non-eigenvalue models).

We shall not discuss the non-trivial history of invention and understanding of all these notions (the crucial steps being discovery of the "multiscaling continuum limits" [19], [20], which preserve integrable structure of discrete models in continuum case; hypothesis of equivalence of quantum and topological $2 d$ gravities [9] and its proof [23], 24], provided by discovery of Kontsevich models [22] as a peculiar and powerful tool for description of topology of the module spaces). Instead, following the mean line of these notes, we shall concentrate on intrinsic relation between (multiscaling) continuum limits and integrability: the notion of continuum limits is in fact built into the theory of integrable hierarchies and the underlying representation theory of Kac-Moody algebras.

In the case of the eigenvalue models the central issue here is the interrelation between Toda-lattice and KP hierarchies, even its more narrow aspect: elimination of the zero-time $N$, present in the Toda-lattice case. From rep- 
resentation theory (or conformal field theory, what is essentially the same) point of view the thing is that the zero-time (which labels the filling level of Dirac sea in the fermionic picture) is associated with the zero-modes of scalar field and its elimination is just the change of boundary conditions which eliminates zero-modes. The simplest example of this "twisting" procedure is just transformation from periodic to antiperiodic scalars - it still preserves possibility to have fermionic description (where it looks like a switch from Ramond to Neveu-Schwarz sector), and thus does not take us out of the field of conventional integrable hierarchies. In representation theory one can interpret the same operation just as a switch from the homogeneous to principal representation, which are associated with the Toda-lattice and KP hierarchies respectively.

This remarkably simple description is of course far from obvious, if one investigates continuum limit in naive way, without taking integrable structure into account explicitly, but just sending the number of degrees of freedom in discrete theory (i.e. the matrix size $N$ ) to infinity (together with the inverse lattice spacing, if any). We refer to the classical review [18] for discussion of what are the naive continuum limits in lattice gauge theories, i.e. what are the conditions for getting the second-order phase transitions, which allow for a continuum-like scaling behavious in the vicinity of the critical point, with critical exponents defining all the continuum physics, from quantum dimesnion of the space-time to spectrum of particles. The problem with naive continuum limits is that they can easily destroy integrable structure of the theory (the underlying hidden symmetries), unless special precaution is taken: the critical point (which is in fact a low-codimensional hypersurface in the infinite-dimensional space of parameters) should be approached from the certain directions, so that Ward identities are not explicitly broken.

As soon as this word - Ward -dentities - is pronounced, we already get into the field of integrable systems and the issue can be discussed inside this field. The above-mentioned switch from periodic to antiperiodic fields is of course apparent if the discrete and continuos Virasoro constraints (represented by formulas (1.2) and (1.3) in the Introduction) are compared, but this is a posteriori information, because so far we interpreted "continuous Virasoro constraints" as the Ward-identities for the $V=X^{3}$-Kontsevich model, and it still remains to be explained why Kontsevich model is indeed what arises after continuum limit is taken. The simplest approach to this problem is to make use of the identity between discrete 1-matrix model and Gaussian 
Kontsevich model [56], established in section 3.8 above. Then the $X^{3}$-model arises in the large- $N$ limit just when the matrix integral is evaluated by the steepest descent method [36]. We shall present this simple calculation in the last subsection below, but before we take a somewhat more direct (and complicated) approach in order to reveal at least some of ideas, underlying the entire theory of continuum limits.

\subsection{From Toda-chain to $\mathrm{KdV}$}

We begin with the simplest existing example: continuum limit, in which the lowest equation of the "Volterra hierarchy",

$$
\frac{\partial R_{n}}{\partial t}=-R_{n}\left(R_{n+1}-R_{n-1}\right)
$$

turns into the lowest $\mathrm{KdV}$ equation:

$$
\frac{\partial r}{\partial T_{3}}=-\frac{1}{3} r^{\prime \prime \prime}-2 r r^{\prime}
$$

Volterra hierarchy is a reduction of Toda-chain hierarchy, with $R_{n}=e^{\phi_{n}-\phi_{n-1}}$, arising when all the odd-times $t_{2 k+1}=0$ and all $\phi_{n}$ are supposed to be independent of them. Therefore this hierarchy is clearly related to the discrete 1-matrix model. We"ll turn to the study of 1-matrix model in the next subsection, but here we just address the transformation from (5.1) to (5.2) [103, 26].

The basic idea of taking continuum limit is to change discrete "zero-time" $n$ for continuum variable $x$ (to be after all substituted by $T_{1}$ of the continuous hierarchy). In other words, the idea is to consider a subset of functions $R_{n}$, which satisfy Volterra equation and depend on $n$ very smoothly, so that they can actually be substituted by a smooth function $R(x)$. This is a very natural thing to do, of course, when one is interested in the large- $n$ limit of the equation. Namely, one substitutes (5.1) by

$$
\frac{\partial R(x)}{\partial t}=-R(x)(R(x+\epsilon)-R(x-\epsilon)),
$$

and take the limit $\epsilon \rightarrow 0$, which, after rescaling $x \rightarrow \epsilon x$, gives rise to "Bateman equation",

$$
\frac{\partial R(x)}{\partial t}=-R(x) R^{\prime}(x)
$$


This is a very interesting equation (see 104 for description of the amusements of the related theory, which is in fact intimately related to the theory of jets). However, it is much simpler that $\mathrm{KdV}$ equation (for example, it is completely integrable in the most trivial sense of the word: entire set of solutions, satisfying any boundary conditions can be immediately written down, see $104 \|)$. KdV equation can be considered as a sort of "quantization" of (5.4) (unfortunately this very interesting subject did not yet attract enough attention and is not studied well enough).

Remarkably, Bateman equation is not the only possible limit of Volterra equation: a fine tuning procedure ("double-scaling limit") exist, which can provide less trivial - KdV - equation [103]. Indeed, imagine, that in continuum limit $R_{n}$ tends to a constant $R_{0}$, and the function $r(x)$ arises only as scaling approximation to this constant: $R(x)=R_{0}\left(1+\epsilon^{s} r(x)\right)$. Then the leading term at the r.h.s. of (5.4) is $\epsilon R R^{\prime}(x)=-2 \epsilon^{s} r(x)\left(1+\mathcal{O}\left(\epsilon^{2}, \epsilon^{s}\right)\right)$, and instead of (5.4) we would get:

$$
\frac{\partial r}{\partial t}=-2 \epsilon R_{0} r^{\prime}(x)\left(\left(1+\mathcal{O}\left(\epsilon^{2}, \epsilon^{s}\right)\right)\right.
$$

This equation is even simpler that (5.4) - it is just linear, but in fact it is too simple to preserve its form: by a simple change of variablesto

$$
\begin{array}{r}
\tilde{x}=x-2 \epsilon R_{0} t, \\
\tilde{t}=\epsilon^{3} R_{0} t
\end{array}
$$

it can be transformed into

$$
\frac{\partial r}{\partial \tilde{t}}=\epsilon^{-2} \mathcal{O}\left(\epsilon^{2}, \epsilon^{s}\right)
$$

and terms at the r.h.s. also deserves beeing taken into account. Then we get:

$$
\begin{aligned}
\frac{\partial r(x)}{\partial t} & =-2 \epsilon R_{0}\left(1+\epsilon^{s} r(x)\right)\left(r^{\prime}(x)+\frac{1}{6} \epsilon^{2} r^{\prime \prime \prime}(x)+\mathcal{O}\left(\epsilon^{4}\right)\right)= \\
& =-2 \epsilon R_{0}\left(r^{\prime}(x)+\frac{1}{6} \epsilon^{2} r^{\prime \prime \prime}(x)+\epsilon^{s} r r^{\prime}(x)+\epsilon^{2} \mathcal{O}\left(\epsilon^{2}, \epsilon^{s}\right)\right)
\end{aligned}
$$

\footnotetext{
46 This change of variables is implied by the relation:

$$
\frac{\partial}{\partial t}+2 \epsilon^{s} R_{0} \frac{\partial}{\partial x}=\left(\frac{\partial \tilde{t}}{\partial t}+2 \epsilon^{s} R_{0} \frac{\partial \tilde{t}}{\partial x}\right) \frac{\partial}{\partial \tilde{t}}+\left(\frac{\partial \tilde{x}}{\partial t}+2 \epsilon^{s} R_{0} \frac{\partial \tilde{x}}{\partial x}\right) \frac{\partial}{\partial \tilde{x}}=\frac{\partial}{\partial \tilde{t}} .
$$
}


and, after the change of variables (5.7),

$$
\frac{\partial r(\tilde{x})}{\partial \tilde{t}}=-\frac{1}{3} r^{\prime \prime \prime}(\tilde{x})-2 \epsilon^{s-2} r r^{\prime}(\tilde{x})+\mathcal{O}\left(\epsilon^{2}, \epsilon^{s}\right) .
$$

It is now clear, that the choice $s=2$ is distinguished (a critical point) and at this point we get:

$$
\frac{\partial r}{\partial T_{3}}=-\frac{1}{3} \frac{\partial^{3} r}{\partial T_{1}^{3}}-2 r \frac{\partial r}{\partial T_{1}},
$$

where new notation $T_{1}$ and $T_{3}$ is introduced for $\tilde{x}$ and $\tilde{t}$ respectively. This is already the $\mathrm{KdV}$ equation (5.2), and our conclusion is:

While the naive continuum limit of Volterra equation is just a simple Bateman equation, the scaling limit can be fine tuned so that KdV equation arises instead. The crucial ingredient of this adjustement is the change of time-variables $\{t\} \longrightarrow\{T\}$, which involves singular parameter $\epsilon$. The procedure can be easily generalized to the entire Volterra hierarchy, and fine tuning allows to get the entire KdV hierarchy in the limit of $\epsilon \rightarrow 0$. Usually transformation to the "Kazakov variables" $\{T\}$ (they are a little different from those originally introduced by V.Kazakov in [19]) from $\{t\}$ is some linear triangular transformation.

An important detail is that this procedure requires restriction to only even time-variables $t_{2 m}, m \geq 0$. (If odd times are also involved, a pair of KdV hierarchies arises in the continuum limit - this is not a "minimal" case.) Thus "irreducible" realization of continuum limit requires reduction of original hierarchy. This can be also seen from the fact that the lowest $\mathrm{KdV}$ equation arises from the lowest Volterra equation, which is related to the second eqaution of Toda-chain hierarchy.

Unfortunately this simple piece of theory (continuum limits in terms of hierarchies) has never been worked out in full details (for the entire Todalattice hierarchy, its multicomponent generalizations and their reductions). As we already mentioned, this theory will involve the general relation between homogeneous and principal representations of the (level $k=1$ ) Kac-Moody algebras.

\subsection{Double-scaling limit of 1-matrix model}

Now we proceed to discussion of a slightly different approach to continuum limits, which is directly adjusted to the needs of matrix models. The naive 
idea [20], 22] is to forget about integrability and just look at the Ward identities (Virasoro constraints in the 1-matrix case) and take a continuum limit of these identities. This approach makes close contact with the standard technique of "loop equations" (Makeenko-Migdal equations [105]) in the theory of matrix models, of which Virasoro and $W$-constraints are just particular examples. ${ }^{47}$

However, carefull analysis of continuum limit of discrete Virasoro constraints 28] makes it clear that the procedure is far less simple than one can think in advance (usually derivations are not very carefull and details are just "put under the carpet"). The crucial problem is that we want peculiar (double scaling) rather than naive limit, and, as we mentioned in the previous subsection, this also requires a certain reduction (elimination of the odd-times $t_{2 m+1}$ ). If parity symmetry (w.r.to the change of $H \rightarrow-H$ in the original matrix integral) is taken into account, one can easily throw away first derivatives w.r.to the odd-times $t_{2 m+1}$, just because $\left.\frac{\partial Z_{N}}{\partial t_{2 m+1}}\right|_{t_{2 k+1}=0}=0$, but this is no longer true as far as the second derivatives $\left.\frac{\partial^{2} Z_{N}}{\partial t_{2 m+1} \partial t_{2 l-1}}\right|_{t_{2 k+1}=0}$ are concerned, which appear in (the "quantum piece" of) the Virasoro constraints (1.2). It is a highly non-trivial feature of loop equations (having its origin in their integrable structure!), that in continuum limit these terms can be in fact carefully eliminated. The thing is that the second derivatives of $\log Z_{N}$ appear to be a local objects, in the sense that they depend only on $Z_{\tilde{N}}$ with the difference $|\tilde{N}-N| \leq m+l$, which does $n o t$ blow up as $N \rightarrow \infty$ in continuum limit. Moreover, the differences $\frac{\partial^{2} \log Z_{N}}{\partial t_{2 m+1} \partial t_{2 l-1}}-\frac{\partial^{2} \log Z_{N}}{\partial t_{2 m} \partial t_{2 l}}$ almost tend to zero, leaving some simple (though vitaly important) correction to arising continuous loop equations. This locality property allows one to get rid of these dangerous odd-time derivatives, substituting them just by second derivatives w.r.to the even-times. Since such substitution is possi-

\footnotetext{
${ }^{47}$ One of the puzzles in the theory of non-eigenvalue models is to identify grouptheoretical meaning of generic loop equations: they are usually introduced as equations of motion rather than as Ward identities (see discussion at the beginning of Section 2 above), and thus their implications are more obscure and technical means to deal with them are much more restricted. When group theory description will be found, it will very soon reveal the (generalized) integrable structure of non-eigenvalue models and it will be a big step forward in the whole theory.
} 
ble only for logarithms of $Z_{N}$, continuous constraints appear imposed on the square root of original partition function (or on the $\frac{1}{p}$-th power in the case of the $p$-1-component conformal models). Another aspect of this trick to deal with the odd-time derivatives is that it makes the entire derivation depending on the fact that the theory is integrable - this is what guarantees the above-mentioned locality. Since the way to reveal integrability, by looking at the loop equations themselves is yet not very well understood, the whole calculation becomes not quite self-contained (but of course, if we know everything about integrable structure this is not a real drawback, this is just a limitation of particular approach, starting from the loop equations) . In particular, this is the only loophole, which is still not filled in the description of continuum limit of conformal (multi-component) matrix models, which in all other respects goes in exactly in parallel with the 1-component (1-matrix) case. ${ }^{\text {t8 }}$

We shall now describe briefly the steps of this calculation for the 1-matrix model, refering for all the details to refs. [28] and [45]. Our previous discussion already contain motivations for the main steps, so we do not need to go into detailed explanations. Manipulations below, involving Kazakov variables can look a little artificial, but we repeat that they can be interpreted as a switch from the Toda-type to KP-type hierarchies, which, as we already saw in the previous subsection, is naturally associated with the double-scaling continuum limit.

We start from the discrete Virasoro constraints (1.2), rewritten in terms of a generating functional ("stress tensor" on the spectral plane):

$$
L_{-}(z) Z_{N}=0
$$

where

$$
L_{-}(z)=\sum_{n \geq-1}^{\infty} L_{n} z^{-n-2}=\frac{1}{2}\left(J^{2}(z)\right)_{-},
$$

\footnotetext{
48 It transforms discrete $W$-constraints into continuum $W$-constraints, which in their turn arise from the GKM with the appropriate potential [30], 55. Unfortunately, since the GKM-inteprpretation of discrete multicomponent models (like the one existing in the 1-matrix case, see s.3.8) is yet unknown, the direct way to take their continuum limit - like the one to be described in the next subsection for the 1-matrix case - is also yet unavailable. For more details about conformal matrix models, their integrable structure and continuum limits see refs. [39].
} 
and

$$
\begin{gathered}
J(z)=\partial \phi(z)=\sum_{n=-\infty}^{\infty} J_{n} z^{-n-1} ; \\
\phi(z)=\frac{1}{\sqrt{2}} \sum_{k \geq 0} t_{k} z^{k}-\sqrt{2} \sum_{k>0} \frac{z^{-k}}{k} \frac{\partial}{\partial t_{k}} ; \\
J_{-k}=\sqrt{2} \frac{\partial}{\partial t_{k}} ; \quad J_{k}=\frac{1}{\sqrt{2}} k t_{k}, \quad k \geq 0 ; \\
\frac{\partial}{\partial t_{0}} Z_{N}=N Z_{N} .
\end{gathered}
$$

Next, we need to reduce the original partition function:

$$
Z_{N}\{t\} \longrightarrow Z_{N}^{\text {red }}\left\{t_{\text {even }}\right\} \equiv Z_{N}\left\{t_{\text {odd }}=0, t_{\text {even }}\right\} \text {. }
$$

All odd virasoro generators $L_{2 n+1}$ act trivially on $Z_{N}^{\text {red }}$, since $\left.\frac{\partial Z_{N}}{\partial t_{2 k+1}}\right|_{t_{\text {odd }}=0}=0$, and we need to consider only $L_{2 n}$. Introduce alsoty

$$
\begin{aligned}
\phi^{\text {red }}(z) & \equiv \frac{1}{\sqrt{2}} \sum_{k \geq 0} t_{2 k} z^{2 k}-\sqrt{2} \sum_{k>0} \frac{z^{-2 k}}{k} \frac{\partial}{\partial t_{2 k}} ; \\
L^{\text {red }}(z) & =\frac{1}{2}\left(\partial \phi^{\text {red }}(z)\right)^{2} ; \\
L_{2 n}^{\text {red }} & \equiv \sum_{k>0} k t_{2 k} \frac{\partial}{\partial t_{2 k+2 n}}+\sum_{k=0}^{n} \frac{\partial^{2}}{\partial t_{2 k} \partial t_{2 n-2 k}} .
\end{aligned}
$$

Now we have two issues to be discussed separately. The first one is the change from $t_{2 k}$ to Kazakov variables $T_{2 m+1}$. The second is the difference between constraints imposed on $Z^{\text {red }}$ and $Z$.

${ }^{49}$ Note that $\phi^{\text {red }}(z) \neq\left.\phi(z)\right|_{t_{\text {odd }}=0}$ and similarly $L_{2 n}^{\text {red }}(z) \neq\left. L_{2 n}\right|_{t_{\text {odd }}=0}$ : some factors of 2 in (5.13) being responsible for this discreapancy. In fact $L^{\text {red }}$ are related to generators of the Virasoro constraints in the complex-matrix model [28],

$$
Z_{N}^{C}=\int d M \exp \left(\sum_{k \geq 0} t_{2 k} \operatorname{Tr}\left(M M^{\dagger}\right)^{k}\right)
$$

and in continuum limit $Z_{N}^{C} \sim \sqrt{Z_{2 N}^{\text {red }}}$. 
The simplest way to describe Kazakov variables is to introduce one more - antiperiodic - scalar field,

$$
\Phi(u)=\frac{1}{\sqrt{2}} \sum_{k \geq 0} T_{2 k+1} u^{k+\frac{1}{2}}-\sqrt{2} \sum_{k \geq 0} \frac{u^{-k-\frac{1}{2}}}{k+\frac{1}{2}} \frac{\partial}{\partial \tilde{T}_{2 k+1}} .
$$

Here $\tilde{T}$ and $T$ are related by transfromation

$$
T_{2 k+1}=\tilde{T}_{2 k+1}+\epsilon^{2} \frac{k}{k+\frac{1}{2}} \tilde{T}_{2 k-1}+2 \epsilon N \delta_{k, 0} .
$$

Impose now a relation:

$$
\begin{aligned}
\partial \phi^{\mathrm{red}}(z) & =\frac{1}{\epsilon^{2}} U^{-1} \partial \Phi(u) U \\
z^{2} & =1+\epsilon^{2} u
\end{aligned}
$$

and in continuum limit $\epsilon$ is assumed to vanish. This is a relation which maps homogeneous representations into principal, but its invariant meaning (especially - from the point of view of conformal field theory) does not seem to be enough understood. Anyhow, this relations establishes a relation between $t_{\text {even }}$ and $T$. Namely, comparing the coefficients in front of the positive powers of $u$ at both sides of this equation, we get:

$$
\begin{aligned}
T_{2 k+1} & =\frac{1}{2} \epsilon^{2 k+1} \sum_{m \geq k}^{\infty} \frac{g_{m} \Gamma\left(m+\frac{1}{2}\right)}{(m-k) ! \Gamma\left(k+\frac{3}{2}\right)}, \quad k \geq 0 ; \\
g_{m} & =m t_{2 m}, \quad m \geq 1 ; \quad g_{0}=2 N .
\end{aligned}
$$

Inverse transformation looks like

$$
g_{m}=2 \sum_{k \geq m}(-)^{k-m} \frac{T_{2 k+1} \Gamma\left(k+\frac{3}{2}\right)}{\epsilon^{2 k+1}(k-m) ! \Gamma\left(m+\frac{1}{2}\right)} .
$$

Now,

$$
\frac{\partial}{\partial t_{2 k}}=\frac{1}{2} \sum_{m=0}^{k-1} \frac{\Gamma\left(k+\frac{1}{2}\right) \epsilon^{2 k+1}}{(k-m-1) ! \Gamma\left(m+\frac{3}{2}\right)} \frac{\partial}{\partial \tilde{T}_{2 m+1}},
$$

and using this formula when comparing the negative powers of $u$ we find:

$$
\begin{aligned}
U & =\exp \left(\sum_{m, n} A_{m n} \tilde{T}_{2 m+1} \tilde{T}_{2 n+1}\right), \\
A_{m n} & =2 \frac{(-)^{m+n}}{\epsilon^{2(m+n+1)}} \cdot \frac{\Gamma\left(m+\frac{3}{2}\right) \Gamma\left(n+\frac{3}{2}\right)}{m ! n !(m+n+1)(m+n+2)} .
\end{aligned}
$$


The square of relation (5.16) is:

$$
\left(\partial \phi^{\mathrm{red}}\right)^{2}(z)=\frac{1}{\epsilon^{4}} U^{-1}(\partial \Phi)^{2}(u) U,
$$

or

$$
\sum_{p \geq 0} L_{2 p}^{\text {red }} z^{-2 p-2}=\frac{1}{\epsilon^{4}} U^{-1}\left(\sum_{n \geq-1} \tilde{\mathcal{L}}_{2 n} u^{-n-2}\right) U .
$$

This equality implies that

$$
\begin{gathered}
U^{-1} \tilde{\mathcal{L}}_{2 n} U=\epsilon^{4} \sum_{p \geq 0} L_{2 p}^{\mathrm{red}} \oint_{\infty} \frac{u^{n+1} d u}{z^{2 p+2}}= \\
=\epsilon^{-2 n} \sum_{p=0}^{n+1}(-)^{n+1-p} C_{n+1}^{p} L_{2 p}^{\mathrm{red}}
\end{gathered}
$$

since

$$
\begin{gathered}
\epsilon^{4} \oint_{\infty} \frac{u^{n+1} d u}{z^{2 p+2}}=\oint_{\infty} \frac{u^{n+1} d u}{\left(1+\epsilon^{2} u\right)^{p+1}}=\frac{1}{\epsilon^{2 n}} \frac{\Gamma(-p)}{(n+1-p) ! \Gamma(-n-1)}= \\
=\frac{(-)^{n+p+1}}{\epsilon^{2 n}} \frac{(n+1) !}{p !(n+1-p) !}=\frac{(-)^{n+1-p}}{\epsilon^{2 n}} C_{n+1}^{p} .
\end{gathered}
$$

Explicit expressions for the generators $\tilde{\mathcal{L}}_{2 n}$ (which are harmonics of the stress tensor $\frac{1}{2}(\partial \Phi)^{2}(u)$ of antiperiodic field $\left.\Phi(u)\right)$, are:

$$
\begin{gathered}
\tilde{\mathcal{L}}_{-2}=\sum_{k \geq 1}\left(k+\frac{1}{2}\right) T_{2 k+1} \frac{\partial}{\partial \tilde{T}_{2(k-1)+1}}+\frac{T_{1}^{2}}{4} ; \\
\tilde{\mathcal{L}}_{0}=\sum_{k \geq 0}\left(k+\frac{1}{2}\right) T_{2 k+1} \frac{\partial}{\partial \tilde{T}_{2 k+1}} ; \\
\tilde{\mathcal{L}}_{2 n}=\sum_{k \geq 0}\left(k+\frac{1}{2}\right) T_{2 k+1} \frac{\partial}{\partial \tilde{T}_{2(k+n)+1}}+ \\
+\frac{1}{4} \sum_{k=0}^{n-1} \frac{\partial^{2}}{\partial \tilde{T}_{2 k+1} \partial \tilde{T}_{2(n-k-1)+1}}-\frac{(-)^{n}}{16 \epsilon^{2 n}} ; n>0 .
\end{gathered}
$$

So far everything what was done was just change of variables and all relations were exact for any $\epsilon$, no limits were taken. 
Operators (5.24) are very similar to $\mathcal{L}_{2 n}$, arising in the "continuous Virasoro constraints" (1.3), imposed on partition function of $X^{3}$-Kontsevich model. There are, however, two discreapancies.

First, $\frac{\partial}{\partial \tilde{T}}$ appear in (5.24) instead of $\frac{\partial}{\partial T}$ in generators in (1.3). One can argue that this difference is not really essential, since $\tilde{T}_{2 k+1}$ and $T_{2 k+1}$ differ by terms, which are proportional to $\epsilon^{2}$ and thus vanish in the continuum limit $\epsilon \rightarrow 0$. (Note, however, that this reasoning can be applied only for every particular constraint $\tilde{\mathcal{L}}_{2 n} Z=0, n \geq-1$, not to the entire generating functional, where different terms are summed, multiplied by different powers of $\epsilon$.)

The second discreapancy is a litlle more serious: it is the occurence of an extra term $\frac{(-)^{n+1}}{16 \epsilon^{2 n}}$ for all $n \geq 0$ (this difference is present for $n=0$ as well, because $\mathcal{L}_{0}$ contains the item $\frac{1}{16}$, which is lacking in (5.24).) This extra term can not be eliminated by just taking continuum limit: moreover, it blows up instead of vanishing when $\epsilon \rightarrow 0$. Remarkably enough, this term disappears when we turn to consideration of actual Virasoro constraints, not just a formal choice of time variables. It cancels completely with the other potential source of problem for the derivation of continuous Ward identites. We proceed now to this most sophisticated matter in this whole subsection.

The thing is that, as we already mentioned before, the reduction of discrete Virasoro constraint $L_{2 n} Z_{N}=0$ contains some non-vanishing terms with the odd-time derivatives:

$$
\begin{aligned}
& \left(\sum_{k>0} 2 k t_{2 k} \frac{\partial}{\partial t_{2 k+2 n}}+2 \sum_{k=0}^{n} \frac{\partial^{2}}{\partial t_{2 k} \partial t_{2 n-2 k}}\right) Z_{N}^{\mathrm{red}}= \\
= & \left(\sum_{k=0}^{n} \frac{\partial^{2}}{\partial t_{2 k} \partial t_{2 n-2 k}}-\sum_{k=0}^{n-1} \frac{\partial^{2}}{\partial t_{2 k+1} \partial t_{2 n-2 k-1}}\right) Z_{N}^{\mathrm{red}} .
\end{aligned}
$$

We added an extra term with the second even-time derivatives to both sides of the identity, in order to get at the r.h.s. a combination, which has a chance to vanish in continuum limit. (This formula still needs to be corrected, see eq. (5.29) below.)

In order to find rigorous reason for elimination of the terms at the r.h.s we need to address to explicit formulas from the last subsection of section 4 
(no simpler way is known so far). The crucial formula which we need is:

$$
\frac{\partial^{2} \phi_{n}}{\partial t_{k} \partial t_{l}}=\frac{\partial}{\partial t_{k}} \frac{\left\langle n\left|h^{l}\right| n\right\rangle}{\langle n \mid n\rangle}=\left(\sum_{m>n}-\sum_{m<n}\right) \frac{\left\langle n\left|h^{k}\right| m\right\rangle\left\langle m\left|h^{l}\right| n\right\rangle}{\langle m \mid m\rangle\langle n \mid n\rangle}
$$

and the most important feature of it is its $R$-matrix structure (the fact that a difference occurs at the r.h.s.). This structure implies almost complete cancellation of terms, when we sum over $n$ in order to get $\log Z_{N}=\sum_{0}^{N-1} \phi_{n}$, leaving only a finite sum of the length independent of $\mathrm{N}$ :

$$
\frac{\partial^{2} \log Z_{N}}{\partial t_{k} \partial t_{l}}=\sum_{0<j<\min (k, l)}\left(\sum_{n=N-j}^{N-1} \frac{\left\langle n\left|h^{k}\right| n+j\right\rangle\left\langle n+j\left|h^{l}\right| n\right\rangle}{\langle n \mid n\rangle\langle n+j \mid n+j\rangle}\right) .
$$

The finite sum at the r.h.s. can be expressed in terms of $R_{n}=e^{\phi_{n}-\phi_{n-1}}$, which are exactly the quantites to satisfy equations of Volterra hierarchy and tending to constant (denoted by $R_{0}$ in the previous section) in continuum limit. Locality property - the finiteness of the sum at the r.h.s in (5.26) implies that this r.h.s. tend to a constant value as $N \rightarrow \infty$. This constant does not completely cancels in the difference

$$
\left(\sum_{k=0}^{n} \frac{\partial^{2}}{\partial t_{2 k} \partial t_{2 n-2 k}}-\sum_{k=0}^{n-1} \frac{\partial^{2}}{\partial t_{2 k+1} \partial t_{2 n-2 k-1}}\right) \log Z_{N}^{\mathrm{red}}
$$

and the remaining contributions appears to be exactly what necessary to cancel the dangerous term $\frac{(-)^{n+1}}{16 \epsilon^{2 n}}$ which appeared in the difference between $\tilde{\mathcal{L}}_{n}$ and $\mathcal{L}_{n}$. We refer to ref. 28 for more details about these cancellations, and the only thing to discuss at the rest of this subsection is the difference between the r.h.s. of (5.25) and (5.28). In the second expression the second derivatives are taken of $\log Z$, while they are of $z$ itself in the first one. Of course,

$$
\frac{\partial^{2} \log Z_{N}^{\mathrm{red}}}{\partial t_{2 k+1} \partial t_{2 n-2 k-1}}=\frac{1}{Z_{N}^{\mathrm{red}}} \frac{\partial^{2} Z_{N}^{\mathrm{red}}}{\partial t_{2 k+1} \partial t_{2 n-2 k-1}}
$$

but this is not true for even derivatives. So, identity (5.25) yet needs to be transformed a little more in order to contain exactly (5.26) at its r.h.s. If 
this is achieved, the l.h.s. acquires additional contribution and turns into

$$
\begin{gathered}
\sum_{k>0} 2 k t_{2 k} \frac{\partial Z_{N}^{\mathrm{red}}}{\partial t_{2 k+2 n}}+\sum_{k=0}^{n}\left(2 \frac{\partial^{2} Z_{N}^{\mathrm{red}}}{\partial t_{2 k} \partial t_{2 n-2 k}}-\frac{1}{Z_{N}^{\mathrm{red}}} \frac{\partial Z_{N}^{\mathrm{red}}}{\partial t_{2 k}} \frac{\partial Z_{N}^{\mathrm{red}}}{\partial t_{2 n-2 k}}\right)= \\
=4 \sqrt{Z_{N}^{\mathrm{red}}} L_{2 n}^{\mathrm{red}} \sqrt{Z_{N}^{\mathrm{red}}} .
\end{gathered}
$$

As a result of all this reasoning we conclude that the double scaling continuum limit of reduced 1-matrix model can be described by the following relation:

$$
\lim _{\text {d.s. } \epsilon \rightarrow 0, N \rightarrow \infty} \sqrt{Z_{N}^{\text {red }}\left\{t_{\text {even }}\right\}}=U^{-1} Z_{V=\frac{X^{3}}{3}}\{T\},
$$

where factor $U$ is defined in (5.20), relation between $t$ and $T$-variables is given by (5.17) and $Z_{V=\frac{X^{3}}{3}}\{T\}$ is $X^{3}$-Kontsevich model. The motivation for this conclusion is that both sides of the equation satisfy the same continuous Virasoro constraints (1.3).

This whole derivation can be straightforwardly generalized to the case of multiscaling limit in conformal matrix models and analogous relation contains roots of the $p$-th degree, see [39] for detailed discussion.

\subsection{From Gaussian to $X^{3}$ Kontsevich model}

We shall now abandon these complicated matters and give a simple illustration of how the things can work, if expressed in the adequate terms. Namely, as alternative to the sophisticated procedure, involving explicit switch to Kazakov variables and the study of limits of Ward-identites (loop equations), we shall just use the equivalence of the discrete 1-matrix model and Gaussian Kontsevich model, proved in the section 3.8 above in order to take the continuum limit just of this simplest Kontsevich model. This procedure, suggested in ref. 36] appears to be just a kind of a standard eveluation of the integral in the large $N$-limit by the steepest descent method. It is important here that GKM is not sensitive to the size of the matrix $n$ in Kontsevich integral, therefore this limit, when expressed in terms of GKM, has nothing to do with the infinitely large matrices.

Relation to be proved below is

$$
\lim _{\text {d.s. } N \rightarrow \infty} \mathcal{F}_{\{\hat{V}\}}=\mathcal{F}_{\{V\}}^{2},
$$


where $\hat{V}(X)=\frac{1}{2} X^{2}-N \log X$ and $V(X)=\frac{1}{3} X^{3}$.

Very naively, what happens as $N \rightarrow \infty$ is that in the Kontsevich integral,

$$
\int d X \exp \operatorname{tr}\left(-\frac{1}{2} X^{2}+N \log X+\Lambda X\right)
$$

a stationary point arises at $X=X_{0}$, such that

$$
X_{0}=\frac{N}{X_{0}}+\Lambda \text {. }
$$

Expansion of this action in powers of $\tilde{X}=\gamma^{-1}\left(X-X_{0}\right)$ comes entirely from the logarithmic piece:

$$
\begin{aligned}
S-S_{0} & =\frac{\gamma^{2}}{2} \tilde{X}^{2}-N\left(\log \left(1+\frac{\gamma \tilde{X}}{X_{0}}\right)-\frac{\tilde{X}}{X_{0}}\right)= \\
& =\frac{\gamma^{2}}{2}\left(1+\frac{N}{X_{0}^{2}}\right) \tilde{X}^{2}+\sum_{k \geq 3} \frac{N}{k}\left(-\gamma \frac{\tilde{X}}{X_{0}}\right)^{k} .
\end{aligned}
$$

In the continuum limit $\gamma$ should be adjusted in such a way, that quadratic term is finite, i.e. $\gamma \sim\left(1+\frac{N}{X_{0}^{2}}\right)^{-1 / 2}$. Now, if $\Lambda$ remains finite as $N \rightarrow \infty$, $X_{0} \sim \sqrt{N}, \gamma \sim 1$ and all the terms with $k \geq 3$ in the sum are damped as $\gamma^{k} N X_{0}^{-k} \sim N^{1-\frac{k}{2}}$. This is the naive continuum limit. However, it is clear, that one can usually ask $\Lambda$ to behave more adequately - blow up together with growth of $N$ - and fine tune the way in which it tends to infinity so that at last the first term with $k=3$ also survives. For this purpose $\Lambda$ and thus $X_{0}$ should scale in such a way, that both quantites $\gamma^{2}\left(1+\frac{N}{X_{0}^{2}}\right)$ and $\frac{N \gamma^{3}}{X_{0}^{3}}$ remain finite. This requirement in the case of the latter expression means that $\gamma \sim X_{0} N^{-1 / 3}$ and then

$$
\gamma^{2}\left(1+\frac{N}{X_{0}^{2}}\right) \sim \frac{N+X_{0}^{2}}{N^{2 / 3}}
$$

This is never finite, unless $N+X_{0}^{2} \rightarrow 0$ as $N \rightarrow \infty$. This in turn implies that $X_{0} \sim i \sqrt{N}$ and $\Lambda \rightarrow 2 X_{0} \sim 2 i \sqrt{N}$ should be pure imaginary. One can also check that the terms with $k>3$ in the sum (5.34) all tend to zero in this specific limit. Thus we are left with a model which has only cubic and quadratic terms in the action. By simple shift of variables quadratic term 
can be changed for a linear one and we get a description of the theory in the vicinity of the stationary point in terms of an $X^{3}$-Kontsevich model.

In practice things are a little more complicated, because also reduction to even-times should be taken into account. However, this does not really add too many new problems. We need that only even times $t_{2 k}=\frac{1}{2 k} \operatorname{tr} \frac{1}{\Lambda^{2 k}}$ remain non-vanishing, while all the odd times $t_{2 k+1}=\frac{1}{2 k+1} \operatorname{tr} \frac{1}{\Lambda^{2 k+1}}=0$. This obviously implies that the matrix $\Lambda$ should be of block form:

$$
\Lambda=\left(\begin{array}{cc}
\mathcal{M} & 0 \\
0 & -\mathcal{M}
\end{array}\right)
$$

and, therefore, the matrix integration variable is also naturally decomposed into block form:

$$
X=\left(\begin{array}{ll}
\mathcal{X} & \mathcal{Z} \\
\mathcal{Z} & \mathcal{Y}
\end{array}\right)
$$

Then

$$
\begin{gathered}
\mathcal{F}_{\left\{\hat{V}=X^{2} / 2-N \log X\right\}}=\int d \mathcal{X} d \mathcal{Y} d^{2} \mathcal{Z} \\
\operatorname{det}\left(\mathcal{X Y}-\overline{\mathcal{Z}} \frac{1}{\mathcal{Y}} \mathcal{Z} \mathcal{Y}\right)^{N} e^{-\operatorname{tr}\left\{|\mathcal{Z}|^{2}+\mathcal{X}^{2} / 2+\mathcal{Y}^{2} / 2-\mathcal{M X}+\mathcal{M Y}\right\}} .
\end{gathered}
$$

To take the limit $N \rightarrow \infty$, one should assume certain scaling behaviour of $\mathcal{X}$, $\mathcal{Y}$ and $\mathcal{Z}$. Moreover, our previous naive consideration gave us some feeling of the fine tuned scaling behaviour can look like. So we take

$$
\begin{gathered}
\mathcal{X}=\gamma(i \beta I+x), \\
\mathcal{Y}=\gamma(-i \beta I+y), \\
\mathcal{Z}=\gamma \zeta \\
\mathcal{M}=\gamma^{-1}(i \alpha I+m)
\end{gathered}
$$

with some large real $\alpha, \beta$ and $\gamma$. If expressed through these variables, the 
action becomes:

$$
\begin{gathered}
\operatorname{tr}\left(|\mathcal{Z}|^{2}+\mathcal{X}^{2} / 2+\mathcal{Y}^{2} / 2-\mathcal{M} \mathcal{X}+\mathcal{M} \mathcal{Y}-N \log \left(\mathcal{X Y}-\overline{\mathcal{Z}} \frac{1}{\mathcal{Y}} \mathcal{Z} \mathcal{Y}\right)\right)= \\
=\gamma^{2} \operatorname{tr}\left(\frac{1}{2}(i \beta I+x)^{2}+\frac{1}{2} \operatorname{tr}(i \beta I-y)^{2}+|z|^{2}\right)- \\
\quad-\operatorname{tr}(i \alpha I+m)(2 i \beta I+x-y)- \\
-N \operatorname{tr} \log \beta^{2} \gamma^{2}\left(1-i \frac{x-y}{\beta}+\frac{x y}{\beta^{2}}-\frac{|\zeta|^{2}}{\beta^{2}}(1+\mathcal{O}(1 / \beta))\right)= \\
=\left[2 \alpha \beta-\beta^{2} \gamma^{2}-2 N \log \beta \gamma\right] \operatorname{tr} I-2 i \beta \operatorname{tr} m+ \\
+i\left(\beta \gamma^{2}-\alpha+\frac{N}{\beta}\right)(\operatorname{tr} x-\operatorname{tr} y)+\frac{1}{2}\left(\gamma^{2}-\frac{N}{\beta^{2}}\right)\left(\operatorname{tr} x^{2}+\operatorname{tr} y^{2}\right)+ \\
+\left(\gamma^{2}+\frac{N}{\beta^{2}}\right) \operatorname{tr}|\zeta|^{2}- \\
-\operatorname{tr} m x+\operatorname{tr} m y+\frac{i N}{3 \beta^{3}} \operatorname{tr}\left(x^{3}-y^{3}\right)+ \\
+\mathcal{O}\left(N / \beta^{4}\right)+\mathcal{O}\left(|\zeta|^{2} \frac{N}{\beta^{3}}\right)
\end{gathered}
$$

We want to adjust the scaling behaviour of $\alpha, \beta$ and $\gamma$ in such a way that only the terms in the line $(D)$ survive. This goal is achieved in several steps.

The line $(A)$ describes normalization of functional integral, it does not contain $x$ and $y$. Thus, it is not of interest for us at the moment.

Two terms in the line $(B)$ are eliminated by adjustment of $\alpha$ and $\gamma$ :

$$
\gamma^{2}=\frac{N}{\beta^{2}}, \alpha=\frac{2 N}{\beta} .
$$

As we shall see soon, $\gamma^{2}=N / \beta^{2}$ is large in the limit of $N \rightarrow \infty$. Thus, the term $(C)$ implies that the fluctuations of $\zeta$-field are severely suppressed, and this is what makes the terms of the second type in the line $(E)$ negligible. More general, this is the reason for the integral $Z_{\{\hat{V}\}}$ to split into a product of two independent integrals leading to the square of partition function in the limit $N \rightarrow \infty$ (this splitting is evident as, if $\mathcal{Z}$ can be neglected, the only mixing term $\log \operatorname{det}\left(\begin{array}{ll}\mathcal{X} & \mathcal{Z} \\ \mathcal{Z} & \mathcal{Y}\end{array}\right)$ turns into $\left.\log \mathcal{X} \mathcal{Y}=\log \mathcal{X}+\log \mathcal{Y}\right)$. 
Thus, we remain with a single free parameter $\beta$ which can be adjusted so that

$$
\begin{array}{r}
\frac{\beta^{3}}{N} \rightarrow \text { const as } \quad N \rightarrow \infty \\
\text { (i.e. } \left.\beta \sim N^{1 / 3}, \gamma^{2} \sim N^{1 / 3}, \alpha \sim N^{2 / 3}\right),
\end{array}
$$

making the terms in the last line $(E)$ vanishing and the third term in the line $(D)$ finite.

This proves the statement (5.31) in a rather straightforward way. Unfortunately no generalization of this procedure for other discrete models is found so far, the main problem beeing identification of GKM-type realizations of other (for example, conformal) discrete matrix models.

\section{Conclusion}

We came to the end of our brief review of the facts, that are already known about the relation between matrix models and integrable hierarchies. There are still several topics, which are already discussed in the literature, but not presented in these notes.

First of all, we did not discuss the relation between matrix models and theories of topological (Landau-Ginzburg) gravity (LGG). This field is fastly developing during the last months and will be soon ready to inclusion in this kind of reviews. The list of things which are already clarified enough, includes realization of the Ward identities in the form of "recursion relations" for topological gravity [9]. Also the relation between quasiclassical hierarchies, arising in the spherical approximation to topological theories 96, to integrable structure of Generalized Kontsevich model is more or less understood [40]. Of special importance is the chapter of this theory, which provides matrix-model description of module spaces, associated with Riemann surfaces [106, 222]. What still deserves better understanding is axionatic construction of topological gravity, similar to remarkably simple construction of topological LG models (before they are coupled to $2 d$ gravity) in terms of the Grothendieck residues and chiral rings [107: see [108 for a very nice presentation of the latter case and [17] for the first big steps towards similar

construction in the former case. Also relation to the theory of non-conformal 
LG models [109 deserves being clarified. A piece which is essentially lacking so far is the clear description of minimal $(p, q)$-models, coupled to $2 d$ gravity in the case of $p \neq 1$. In this situation Generalized Kontsevich model is known to describe nothing more but duality transformation between $(p, q)$ and $(q, p)$ models [41, rather than the models themselves. This subject is also connected with the theory of Kac-Schwarz operator 110. The work in this direction is extremely important for the understanding of unification of various string models and of essential symmetries of the future string field theory (in particular generic BRST and Batalin-Vilkovisky symmetries are very close analogues of the complete sets of the Ward identites, as described in the general framework in the beginning of section 2). All these things would constitute a natural next section of these notes, but we choosed to wait a little longer untill further clarification is achieved in this fragment of the theory.

Second, we did not touch at all physical interpretations of matrix models, which include quantum gravity, Yang-Mills theory and many other possible applications. This should be a subject of very different reviews, for which the whole content of these notes is just a piece of techniques involved in the study of physical phenomena.

Third, the biggest terra incognita in this branch of science, which remained beyond the scope of these notes, is the theory of non-eigenvalue matrix models, which are related to physical theories in space-time dimensions $d \geq 2$. It is indeed a terra incognita, at least from the point of view of the semi-rigorous analysis, which we are reviewing. The recent breakthrough in this field is due to appearence of the Kazakov-Migdal model [25] (see also the lates review [111] and refernces therein), which for the first time opened the possibility to treat a wide class of non-eigenvalue models by exact methods of localization theory (other names for this field, which in fact is growing up into generic theory of integability are Duistermaat-Heckman theorem or Fourier analysis on group manifolds). The work in this direction is, however, only at the early stages and this is why we decided not to present the first non-systematized results in these notes. A part of it which is very close to beeing satisfactrily understood is the "boudary model" of $c=1$ string (" $d=2$ dilaton gravity") - a very important one from the point of view of general string theory. For the present state of knowledge about this model see [112], and its relation to integrability theory is partly revealed in refs. [113] and [11]. 
In the domain, which was actually reviewed the weakest points are the theory of continuum limits and that of the multicomponent hierarchies. These theories, when developed, can also help to move in the most important direction, which was many time mentioned in the text above: towards creation of more general theory of integrability. The next natural step, when approached from this side should be generalization of conventional integrable hierarchies, which would lift the restriction to level $k=1$ simply-laced KacMoody algebras and unitary representations. The emerging theory will of course have much to do with both localization theory and non-eigenvalue matrix models, and when it is created we shall find ourselves at a new level of understanding, which will be one step closer to the goal of construction of the entire building of string theory (mathematical physics) and will probably provide us with unexpected new means for investigation of the features of the real physical world around us.

\section{Acknowledgements}

These notes reflect the content of the lectures, given at the University of Amsterdam and NIKHEF in February-March 1993. I am deeply indebted to colleagues at Amsterdam for their patience and attention and especially to Sander Bais for his hospitality and encouragement.

It is a pleasure to thank my collaborators and friends, mentioned by names in the end of the Introduction for the lessons that they taught me during our work on the subject of matrix models.

I also aknowledge the support of FOM during the time when these notes were being written.

\section{References}

[1] A.Morozov, UFN 162 \#8 (1992) 84 (Soviet Physics Uspekhi 35 (1992) 671)

[2] I.Klebanov and A.Polyakov, Mod.Phys.Lett. A6 (1991) 3273.

[3] E.Witten, Nucl.Phys. B373 (1992) 187. 
[4] A.Marshakov, A.Mironov, M.Olshanetsky et al. $c=r_{G}$ Theories of $W_{G^{-}}$ Gravity: the Set of Observables as a Model of Simply-Laced G, Preprint ITEP-M2/92, hep-th/9203043, to appear in Nucl.Phys.B.

[5] A.Alekseev and S.Shatshvili, Nucl.Phys B329 (1989) 719.

[6] A.Gerasimov, D.Lebedev et al. Int.J.Mod.Phys. A6 (1991) 977; A.Morozov, Mod.Phys.Lett.A6 (1991) 1525.

[7] A.Its, A.Izergin, V.Korepin, N.Slavnov et al. HEPTH, Dec.1992.

[8] E.Witten, Comm.Math.Phys 117 (1988) 353; 118 (1988) 411.

[9] E.Witten, Nucl.Phys. B340 (1990) 281; Surveys Diff.Geom. 1 (1991) 243.

[10] R.Dijkgraaf and E.Witten, Nucl.Phys. B342 (1990) 486.

[11] D.Birmingham, M.Blau, M.Rakowski and G.Thompson, Phys.Reports 209 (1991) 129.

[12] A.Belavin, A.Polyakov and A.Zamolodchikov, Nucl.Phys. B241 (1984) 333.

[13] V.Knizhnik, UFN 159 \#3 (1989) 401 (Soviet Physics Uspekhi 32 (1989) 945)

[14] A.Perelomov et al. Complex Geometry and String Theory, VINITI, Moscow, 1990 (English transl.: Springer-Verlag 1991)

[15] B.Feigin and D.Fucks, Funk.Anal.\& Prilozh. 16 \#2 (1982) 247; 17 \#3 (1983) 91;

V.Dotsenko and V.Fateev, Nucl.Phys. B240 (1984) 312;

B.Feigin and E.Frenkel, Comm.Math.Phys. 128 (1990) 161.

[16] A.Gerasimov, A.Marshakov, M.Olshanetsky, S.Shatashvili et al., Int.J.Mod.Phys. A5 (1990) 2495.

[17] A.Losev, Descendants constructed from matter fields and K.Saito higher residue pairing in topological $L-G$ theories coupled to topological gravity, Preprint TPI-MINN, May, 1992; 
A.Losev and I.Polyubin, On Connection between Topological LandauGinzburg Gravity and Integrable Systems, Preprint ITEP/UU-ITP, Dec.1992.

[18] A.Migdal, Phys.Reports 102 (1983) 199.

[19] V.Kazakov, Mod.Phys.Lett. A4 (1989) 2125.

[20] E.Brezin and V.Kazakov, Phys.Lett. 236B (1990) 144; M.Douglas and S.Shenker, Nucl.Phys. B335 (1990) 635;

D.Gross and A.Migdal, Phys.Rev.Lett. 64 (1990) 127.

[21] G.Shabat and V.Voevodski, Sov.Math.Dokl. 39 (1989) 38;

A.Levin et al. Phys.Lett. 243B (1990) 207;

D.-J.Smit, Comm.Math.Phys. 143 (1992) 253

[22] M.Kontsevich, Funk.Anal.\& Prilozh. 25 (1991) 50.

[23] E.Witten, in Proc.of the NYC Conference, June 1991.

[24] A.Marshakov, A.Mironov et al. Phys.Lett. 274B (1992) 280.

[25] V.Kazakov and A.Migdal, Induced QCD at Large N, Preprint PUPT1322, 1992.

[26] A.Gerasimov, A.Marshakov, A.Mironov, A.Orlov et al. Nucl.Phys. B357 (1991) 565

[27] A.Mironov et al. Phys.Lett. 252B (1990) 47;

J.Ambjorn, J.Jurkiewicz and Yu.Makeenko, Phys.Lett. 251B (1990) 517 ;

H.Itoyama and Y.Matsuo, Phys.Lett. 255B (1991) 202.

[28] Yu.Makeenko, A.Marshakov, A.Mironov et al. Nucl.Phys. B356 (1991) 574 .

[29] M.Fukuma, H.Kawai and R.Nakayama, Int.J.Mod.Phys. A6 (1991) 1385 ;

R.Dijkgraaf, E.Verlinde and H.Verlinde, Nucl.Phys. B348 (1991) 565. 
[30] S.Kharchev, A.Marshakov, A.Mironov, A.Zabrodin et al. Phys.Lett. 275B (1992) 311; Nucl.Phys. B380 (1992) 181.

[31] R.Dijkgraaf, Intersection theory, integrable hierarchies and topological field theory, Preprint IASSNS-HEP-91/91, hep-th 9201003.

[32] M.Kontsevich, Comm.Math.Phys. 147 \#1 (1992) 1.

[33] M.Adler and P.van Moerbeke, Comm.Math.Phys. 147 (1992) 25. C.Itzykson and J.-B.Zuber, Combinatorics of the Modular Group II. The Kontsevich Integrals, Preprint SPhT/92-001, to appear in Int.J.Mod.Phys.A.

[34] C.Crnkovic, P.Ginsparg and G.Moore, Phys.Lett. 237B (1990) 196; D.Gross and A.Migdal, Phys.Rev.Lett. 64 (1990) 717; E.Brezin, M.Douglas, V.Kazakov and S.Shenker, Phys.Lett. 237B 43.

[35] L.Chekhov and Yu.Makeenko, Phys.Lett. 278B (1992) 271.

[36] S.Kharchev, A.Marshakov, A.Mironov et al. Generalized Kontsevich Model versus Toda Hierarchy and Discrete Matrix Models, Preprint ITEP-M3/92, hep-th/9203043, to appear in Nucl.Phys.B.

[37] M.Douglas, Phys.Lett. 238B (1990) 176.

[38] A.Marshakov, A.Mironov et al. Mod.Phys.Lett. A7 (1992) 1345.

[39] A.Marshakov, A.Mironov et al. Phys.Lett. 265B (1991) 99;

A.Mironov and S.Pakuliak, Double-Scaling Limit in the Matrix Models of a New Type, Preprint FIAN/TD/05-92;

S.Kharchev, A.Marshakov, A.Mironov, S.Pakuliak et al. Conformal Matrix Models as an Alternative to Conventional Multimatrix Models, Preprint ITEP-M4/92, hep-th/9208044, to appear in Nucl.Phys.B.

[40] S.Kharchev, A.Marshakov, A.Mironov et al. Landau-Ginzburg Topological Theories in the Framework of GKM and Equivalent Hierarchies, Preprint ITEP-M5/92, hep-th/9208046.

[41] S.Kharchev and A.Marshakov, Topological versus Non-Topological Theories, Preprint FIAN/TD-15/92. 
[42] E.Date, M.Jimbo, M.Kashiwara and T.Miwa, Transformation Groups for Soliton Equations, RIMS-394, February, 1982.

[43] E.Witten, Two-Dimensional Gauge Theories Revisited, Preprint IASSNS-HEP 92/15.

[44] A.Niemi and O.Tirkkonen, On Exact Evaluation of Path Integrals, Preprint UU-ITP, 3/93.

[45] A.Gerasimov, Yu.Makeenko, A.Marshakov, A.Mironov, A.Orlov et al. Mod.Phys.Lett. A6 (1991) 3079.

[46] E.Brezin, C.Itzykson, G.Parisi and J.-B.Zuber, Comm.Math.Phys. 59 (1978) 35 .

[47] I.Frenkel and V.Kac, Inv.Math. 62 (1980) 23;

G.Segal, Comm.Math.Phys. 80 (1981) 301.

[48] M.Bershadsky and H.Ooguri, Comm.Math.Phys. 126 (1989) 49.

[49] M.Bershadsky, Comm.Math.Phys. 139 (1991) 71

[50] F.A.Bais, T.Tjin and P.van Driel, Nucl.Phys. B357 (1991) 632.

[51] L.Feher, L.O'Raifeartaigh, O.Ruelle, I.Tsutsui and A.Wipf, On the General Structure of Hamiltonian Reductions of WZW Theory, Preprint DIAS-STP-91, UdeM-LPN-TH-71/91.

[52] A.Zamolodchikov, Theor.and Math.Phys. 63 (1985) 1205;

S.Lukianov and V.Fateev, Int.J.Mod.Phys. A3 (1988) 507.

[53] L.Alvarez-Gaume et al., Preprint CERN (1991).

[54] D.Gross, M.Newman, Phys.Lett. 266B (1991) 291.

[55] A.Mikhailov, Preprint ITEP-UU, March 1993.

[56] L.Chekhov and Yu.Makeenko, Phys.Lett. 278B (1992) 271.

[57] E.Gava and K.Narain, Phys.Lett. 263B (1991) 213.

[58] M.Shifman, A.Turbiner et al. Int.J.Mod.Phys. 5A (1990) 2953. 
[59] A.Migdal, Exact Solution of Induced Lattice Gauge Theory at Large N, Preprint PUPT-1323, June, 1992.

[60] C.Itzykson, J.-B.Zuber, J.Math.Phys. 21 (1980) 411.

[61] Harish-Chandra, Amer.J.Math. 79 (1957) 87.

[62] M.Semenov-Tyan-Shanskii, Izv.Akad.Nauk USSR 40 (1976) 562.

[63] A.Alekseev, L.Faddeev and S.Shatashvili, Journal of Geometry and Physics (1989), v.3.

[64] J.Duistermaat and G.Heckman, Invent.Math. 69 (1982) 259.

[65] M.Blau, E.Keski-Vakkuri and A.Niemi, Phys.Lett. B246 (1990) 92;

E.Keski-Vakkuri, A.Niemi, G.Semenoff and O.Tirkkonen, Phys.Rev. D44 (1991) 3899;

A.Hietamaki, A.Niemi, K.Palo et al. Phys.Lett. 263B (1991) 417;

A.Niemi, K.Palo et al. Phys.Lett. 271B (1991) 365; Nucl.Phys. B377 (1992) 295.

[66] I.Kogan, G.Semenoff, N.Weiss et al., Area Law and Continuum Limit in "Induced QCD", Preprint UBCTP 92-26/ITEP M6/92, hep-th/9208012, to appear in Nucl.Phys.B.

[67] A.Morozov, Pair Correlator in the Itzykson-Zuber Integral, Preprint ITEP-M10/92, September, 1992; hep-th/9209074, to appear in Mod.Phys.Lett. A.

[68] S.Shatashvili, Correlation Functions in the Itzykson-Zuber Model, Preprint IASSNS-HEP-92/61, September 1992.

[69] L.Vinet et al. q-Hypergeometric Functions in the Free Field Formalism, Preprint ITEP-M9/92, August 1992.

[70] T.Miwa, Proc.Japan Acad. 58 (1982) 9;

S.Saito, Phys.Rev.Lett. 59 (1987) 1798; Phys.Rev. D36 (1987) 1819.

[71] M.Caselle, A.D'Adda and S.Panzer, Exact Solution of D=1 KazakovMigdal Induced Gauge Theory, Preprint DFTT-38/92, July, 1992. 
[72] I.Kogan, G.Semenoff, N.Weiss et al. Continuum Limits of "Induced QCD": Lessons of the Gaussian Model at $d=1$ and Beyond, Preprint ITEP-M7/92, UBCTP 92-27, August, 1992, to appear in Int.J.Mod.Phys. A.

[73] M.Olshanetsky and A.Perelomov, Phys.Reports.71, \#5 (1981).

[74] P.Freund and A.Zabrodin, Phys.Lett. 284B (1992) 283; Comm.Math.Phys. 147 (1992) 277;

A.Zabrodin, Mod.Phys.Lett. A7 (1992) 441.

[75] P.Freund and A.Zabrodin, Excitation Scattering in Integrable models and Hall-Littlewood-Kerov Polinomials, Preprint hepth-9208063.

[76] A.Turbiner, Funk.Anl.\& Prilozh. 22 (1988) 33; Comm.Math.Phys. 118 (1988) 467.

M.Shifman and A.Turbiner, Comm.Math.Phys. 120 (1989) 347.

[77] A.Perelomov, A.Rosly, M.Shifman, A.Turbiner et al. Int.J.Mod.Phys. 5A (1990) 803.

[78] M.Halpern and E.Kiritsis, Mod.Phys.Lett.4A (1989) 1973. M.Halpern, E.Kiritsis, N.Obers, M.Porrati and J.Yamron, Int.J.Mod.Phys. 5A (1990) 2275.

[79] A.Niemi and P.Pasanen, Phys.Lett.253B (1991) 349.

[80] A.Polychronakos, Nucl.Phys.B324 (1989) 597;

J.Leinaas, J.Myrheim, Phys.Rev.B37 (1988) 9286;

L.Brink, T.Hansson, S.Konstein and M.Vasiliev, "The Calogero model

- anyonic representation, fermionic extension and supersymmetry", Preprint USITP-92-14/Göteborg ITP-92-53 January, 1993.

[81] A.Gorsky and N.Nekrasov Hamiltonian Systems of Calogero Type and Two-Dimensional Yang-Mills Theory, Preprint ITEP-20/93, UUITP6/93; March, 1993.

[82] M.Sato, RIMS Kokyuroku 439 (1981) 30;

M.Sato and Ya.Sato, Lect.Not.Num.Appl.Anal.5 (1982) 259;

G.Segal and G.Wilson, Publ.I.H.E.S.61 (1985) 1. 
[83] V.Kac, Infinite Dimensional Lie Algebras, Cambridge University Press, Cambridge, 1985.

[84] A.Orlov and E.Shulman, Lett.Math.Phys. 12 (1986) 171;

P.Grinevich and A.Orlov, in "Problems of Modern Quantum Field Theory", proceedings of Kiev Conf.; eds.A.Belavin et al., Springer-Verlag Berlin, Heidelberg, 1989.

[85] K.de Voos, Nucl.Phys. B375 (1992) 478.

[86] R.Hirota, Direct Methods in Soliton Theory, in Topics in Current Physics 17 (1980) 157 (eds.R.Bullough and P.Caudrey, Springer-Verlag, New York, 1980).

[87] D.Friedan and S.Shenker, Phys.Lett. 175B (1986) 287;

N.Ishibashi, Y.Matsuo amd H.Ooguri, Mod.Phys.Lett. 2A (1987) 119;

L.Alvarez-Gaume, C.Gomez and C.Reina, Phys.Lett. 190B (1987) 55;

E.Witten, Comm.Math.Phys. 113 (1988) 529;

A.Morozov, Phys.Lett. 196B (1987) 325.

[88] M.Jimbo, T.Miwa and M.Sato, Holonomic Quantum Fields, I-V: Publ.RIMS, Kyoto Univ., 14 (1978) 223; 15 (1979) 201, 577, 871, 1531.

[89] S.Kharchev, A.Marshakov, A.Mironov, A.Orlov and A.Zabrodin, Nucl.Phys.B366 (1991) 569.

[90] K.Ueno and K.Takasaki, Adv.Studies in Pure Math., 4 (1984) 1.

[91] M.Bowick, D.Shewitz et al. Nucl.Phys.B354 (1991) 496.

[92] R.Hirota, Y.Ohta and J.Satsuma, J.Phys.Soc.Japan 57 (1988) 1901.

[93] V.Kac and J.van de Leur, The n-Component KP Hierarchy and Representation Theory, Preprint, January, 1993.

[94] G.Moore, Comm.Math.Phys. 133 (1990) 261.

[95] E.Witten, The $N$ Matrix Model and Gauged WZW models, Preprint IAS-HEP-91/26, to appear in Nucl.Phys.B. 
[96] I.Krichever, Comm.Math.Phys. 143 (1992) 415;

B.Dubrovin, Nucl.Phys. 379B (1992) 627;

[97] K.Takasaki and K.Takebe, SDiff(2) KP Hierarchy, Preprint RIMS-814, October, 1991.

[98] Yu.Makeenko and G.Semenoff, Int.J.Mod.Phys.A6 (1991) 3455;

D.Gross and M.Newman, Phys.Lett. 266B (1991) 291.

[99] P.Di Francesco, C.Itzykson and J.-B.Zuber, Comm.Math.Phys. 151 (1993) 193.

[100] T.Takebe, From General Zakharov-Shabat Equations to the KP and the Toda-Lattice Hierarchies, Preprint RIMS-779, 1991.

[101] E.Date, M.Jimbo, M.Kashiwara and T.Miwa, Transformation Groups for Soliton Equations, RIMS-394, February, 1982.

[102] S.Kharchev et al., Preprint ITEP-M12/92.

[103] S.Novikov, S.Manakov, L.Pitaevski and V.Zakharov, Theory of Solitons, Plenum Press, New York, 1984.

[104] D.Fairlie, J.Govaerts et al. Nucl.Phys.B373 (1992) 214;

D.Fairlie and J.Govaerts, Phys.Lett. 281B (1992) 49; J.Math.Phys. 33 (1992) 3542.

[105] Yu.Makeenko and A.Migdal, Phys.Lett.88B (1979) 135.

[106] R.Penner, Comm.Math.Phys.113 (1987) 299; J.Diff.Geom. 27 (1988) 35.

[107] E.Martinec, Phys.Lett.217B (1989) 431; Criticalities, Catastrophe and Compactigications, in V.Knizhnik memorial volume, 1989;

C. Vafa and N.Warner, Phys.Lett.218B (1989) 51;

B.Greene, C.Vafa and N.Warner, Nucl.Phys.B324 (1989) 371;

W.Lerche, C.Vafa and N.Warner, Nucl.Phys.B324 (1989) 427;

C.Vafa, Mod.Phys.Lett. A6 (1990) 337;

R.Dijkgraaf, E.Verlinde and H.Verlinde, Nucl.Phys.B352 (1991) 59;

K.Li, Nucl.Phys. B354 (1991) 711. 
[108] S.Elitzur, A.Forge and E.Rabinovici, Nucl.Phys.B388 (1992) 131.

[109] S.Cecotti and C.Vafa, Nucl.Phys.B367 (1991) 359.

[110] V.Kac and A.Schwarz, Phys.Lett. B257 (1991) 329.

[111] G.Semenoff and N.Weiss, Symmetry and Observables in Induced QCD, Preprint UBC, March, 1993, to appear in Proceedings of Rakhov School on Mathematical Physics, String Theory and Quantum Gravity at Rakhov, Ukraine, October 1992.

[112] I.Klebanov, in Proc.of 1991 Trieste Spring School on Strings and Quantum Gravity, ed.J.Harvey et al., World Scientific, 1991;

E.Verlinde and H.Verlinde, A Unitary S-Matrix for $2 d$ Black Hole Formation and Evaporation, Preprint PUPT-1380, IASSNS-HEP-93/8, February 1993.

[113] R.Dijkgraaf, G.Moore and R.Plesser, The Partition Function of 2D String Theory, Preprint IASSNS-HEP-92/48, YCTP-P22-92, hep-th 9208031, to appear in Nucl.Phys.B. 